
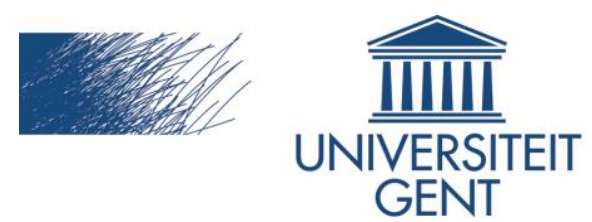

biblio.ugent.be

The UGent Institutional Repository is the electronic archiving and dissemination platform for all UGent research publications. Ghent University has implemented a mandate stipulating that all academic publications of UGent researchers should be deposited and archived in this repository. Except for items where current copyright restrictions apply, these papers are available in Open Access.

This item is the archived peer-reviewed author-version of: Stimuli-responsive nanobubbles for biomedical applications

Authors: Ranhua Xiong, Chaobo Huang, Stefaan De Smedt, Kevin Braeckmans

In: Chemical Society Reviews 50 (9): 5746-5776

To refer to or to cite this work, please use the citation to the published version:

Ranhua Xiong, Chaobo Huang, Stefaan De Smedt, Kevin Braeckmans (2021) Stimuliresponsive nanobubbles for biomedical applications

Chemical Society Reviews 50 (9): 5746-5776

DOI: $10.1039 /$ c9cs00839j 


\title{
Stimuli-responsive Nanobubbles for Biomedical Applications
}

Received 00th January 20xx, Accepted 00th January 20xx DOI: $10.1039 / x 0 x x 00000 x$

\begin{abstract}
Ranhua Xiong ${ }^{a, b}$, Ronald X. Xu ${ }^{\text {d, e }}$, Chaobo Huang* a , Stefaan De Smedt* a, b, c, Kevin Braeckmans*b, c
aJoint Laboratory of Advanced Biomedical Materials (NFU-UGent), College of Chemical Engineering, Nanjing Forestry University (NFU), Nanjing 210037, P. R. China.

'baboratory of General Biochemistry and Physical Pharmacy, Faculty of Pharmaceutical Sciences, Ghent University, 9000 Ghent, Belgium.

'Centre for Advanced Light Microscopy, Ghent University, 9000, Ghent, Belgium.

${ }^{\mathrm{d}}$ Department of Precision Machinery and Precision Instrumentation, University of Science and Technology of China Hefei 230022, P. R. China.

eDepartment of Biomedical Engineering, The Ohio State University, Columbus, OH 43210, USA.

*Kevin.Braeckmans@UGent.be, Stefaan.Desmedt@UGent.be, chaobo.huang@njfu.edu.cn

Stimuli-responsive nanobubbles have received increased attention for their application in spatial and temporal resolution of diagnostic techniques and therapies, particularly in multiple imaging methods, and they thus have significant potential for applications in the field of biomedicine. This review presents an overview of the recent advances in the development of stimuli-responsive nanobubbles and their novel applications. Properties of both internal- and external-stimuli responsive nanobubbles are highlighted and discussed considering the potential features required for biomedical applications. Furthermore, the methods used for synthesis and characterization of nanobubbles are outlined. Finally, novel biomedical applications are proposed alongside the advantages and shortcomings inherent to stimuli-responsive nanobubbles.
\end{abstract}

\section{Introduction}

Nanobubbles (NBs) are typically considered a volume of gas or vapor surrounded by a liquid with a size similar to that of nanoparticles (<1000 nm). ${ }^{1}$ NBs are often encountered in daily life, for example, as the tiny gas bubbles formed by mixing air in cold tap water. ${ }^{2-4}$ The concept of NBs can be traced back to the first discovery of aerogels in the 1930s. ${ }^{5}$ Since then NBs have been used for the removal of pollution from water by flotation, ${ }^{6-}$ 8 for supplying oxygen-rich water to accelerate growth in agriculture, ${ }^{9,} 10$ for improving fuel combustion efficiency, ${ }^{11}$ and for saving energy by reducing flow resistance. ${ }^{12-14}$ Furthermore, especially in the last decade, NBs have found application in

a. Address here.

b. Address here.

c. Address here.

† Footnotes relating to the title and/or authors should appear here.

Electronic Supplementary Information (ESI) available: [details of any supplementary

information available should be included here]. See DOI: 10.1039/x0xx00000x biomedical diagnosis and therapy, especially NBs that are responsive to light or ultrasound triggers. ${ }^{15,} 16$ NBs have attracted such considerable attention due to their excellent and unique properties such as small size, high surface-to-volume ratio, longevity, electrostatic charge properties, surface adsorption, and acoustic properties. ${ }^{17}$ Given the increasing interest in NB technology, its theory, synthesis, and applications have been discussed in previous reviews. ${ }^{1,18-22}$

With regard to the biomedical applications, NBs exhibit particularly interesting stimuli-responsive properties to environmental or external triggers, such as ultrasound waves, light, or changes in $\mathrm{pH}$. These unique properties enable a variety of biomedical applications, such as ultrasound imaging, drug delivery, and therapy. While previous reviews have summarized the theory, synthesis, and applications of NBs, they do not reflect the current state of the art in NB research. An updated 
review is needed since a vast number of new research findings in this field have been reported in the past 4 to 5 years (Fig. 1). In this review, we begin with an introduction of the history of both long-living and transient NBs. Next, we introduce the properties of stimuli-responsive NBs which are closely associated with the emerging biomedical applications. We also discuss stimuli-responsive NBs that are triggered by externally applied stimuli (e.g., ultrasound waves, light stimuli, mechanical forces, temperature changes, and electric and magnetic fields), endogenous stimuli such as $\mathrm{pH}$, or multimodal stimuli. We further review the methods for synthesis and characterization of stimuli-responsive NBs and discuss various biomedical applications where stimuli-responsive NBs are used. Finally, the we discuss future perspectives and research directions in NB technology.

\section{History of NBs}

The term "nanobubbles" was first used 20 years ago in the scientific literature and patents (Fig. 1). Our literature search based on the ISI Web of Knowledge identified only two articles relevant to 'nanobubbles' published in 1999. Our patent search of Espacenet identified the first patent citing the keyword 'nanobubble' was published in 2003. Although an identifiable community of researchers working on NBs has been active only over a relative short period, this research field has steadily attracted increasing interest in the recent years, with more than $50 \%$ of the relevant papers and patents published in the last 5 years alone (from 2015 to 2019). The recent boom in research publications in the field of NBs is likely attributed to the achievement of several major milestones in the field, such as the first direct recording of NB images by atomic force microscopy (AFM) in the year 2000.23, 24 Importantly, in the titles of the latter two articles, the bubble is explicitly described in nanoscale, and the term 'nanobubble' was used for the first time, although previous researchers hypothesized that a submicroscopic bubble could exist. Subsequently, NBs were first explored as ultrasound contrast agents in 2003 and 2004. ${ }^{25,} 26$ These initial milestone publications likely convinced researchers that NBs actually existed and had the potential for unique applications, which in turn stimulated more and more interest in this field and resulted in a significant increase in publication of articles and patents (Fig. 1).
In this review, NBs are divided into long-living NBs and transient NBs based on their life-span. Typically, long-living NBs are metastable and are able to last for a few minutes to even a few months. Transient NBs are, instead, unstable with a lifetime between tens of nanoseconds to a few seconds, depending on the external triggering energy.

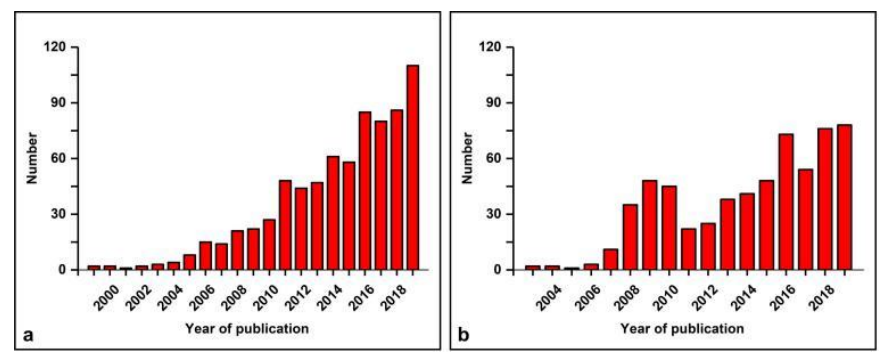

Fig. 1. The annual number of published articles (a) and patents (b) on nanobubbles over the last 20 years, according to the ISI Web of Knowledge and Espacenet, respectively.

As suggested by Seddon et al., long-living NBs could be classified into surface NBs and bulk NBs in accordance with their properties and synthetic processes (Fig. 2). ${ }^{18}$ Surface NBs are described as gas-filled pockets on a surface in the form of a spherical cap (left panel in Fig.

2). The height of the nanobubbles is generally between 10 and 100 $\mathrm{nm}$. Conversely, bulk NBs are stable spherical packages of gas within a liquid with a diameter of less than $1000 \mathrm{~nm}$ (middle panel in Fig. 2).

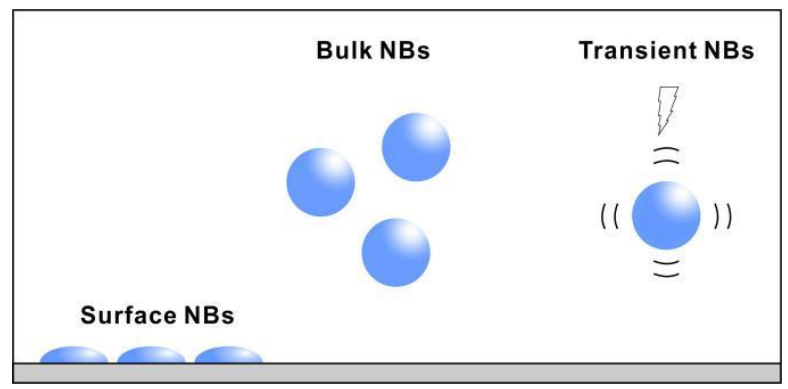

Fig. 2. Three categories of NBs.

Apart from long-living NBs, transient NBs also exist, and are especially relevant for biomedical applications. ${ }^{27-30}$ Typically, transient NBs can be generated by the local deposition of energy in a short time by applying electric, acoustic, or laser pulses in a liquid medium (right panel in Fig. 2) leading to cavitation or boiling. ${ }^{31-34}$ The rapid growth and sudden collapse of transient NBs may result in the quick build-up of high-energy density, high local temperature, or high local pressure. Nonetheless, the overall liquid medium environment remains constant at ambient conditions given the abrupt change induced by the NBs is a very localized effect. This unique property of transient NBs has attracted considerable attention for different applications. For example, these NBs can be used to enhance the 
chemical reactions within liquids or to propagate the generation of free radicals due to dissociation of molecules trapped in the bubbles. ${ }^{35}$ The generation of highly localized mechanical forces by NBs triggered by pulsed laser light has also found biomedical application, such as for local ablation of biological tissues or removal of bacteria. ${ }^{36,37}$

\subsection{History of long-living NBs}

Two types of stable NBs have been discovered which have a long lifetime. The first type of such long-living NBs are surface NBs, first discovered in the 1980s when strong attraction forces between hydrophobic surfaces immersed in water were observed over a range longer than what could be explained by classical van der Waals forces. In further studies it was found that this was due to submicroscopic bubbles which bridged the hydrophobic surfaces. ${ }^{23}$ ${ }^{24}$ These early findings marked the onset of research on NBs, as can be seen from the rapid increase in the number of relevant publications from then on (Fig. 1). To date, several research areas on surface NBs exist, which can be summarized as follows: (1) fundamental characterization of surface NBs, including measuring physical characteristics of surface NBs, such as surface tension, and examining stability in the presence of electrolytes and surfactants; (2) technological development for generating surface NBs, including direct immersion of a hydrophobic substrate into water, temperature increase or pressure reduction at the surface of the substrate, photochemical or electrochemical reactions, and frequently used solvent exchange techniques; (3) development of theories for describing surface NBs, including contamination theory, dynamic equilibrium theory, and diffusive dynamics of pinned surface NBs; and (4) applications of surface NBs, such as solvent exchange for effective cleaning of silicon wafers in the electronics industry. We kindly refer the interested reader to previous review articles on surface NBs for more details on this particular topic. ${ }^{19,22}$ $38-41$

Apart from surface NBs, research on bulk NBs has happened in parallel, which could be divided into the following phases: (1) early discoveries on bulk NBs since the 1960s; (2) a rapid increase in the number of reports on their production and characterization since 2000; and (3) a rapid increase in the number of applications in the past decade. Recently, many bulk NB applications have emerged in various fields. First of all, because of their long lifetime and compressibility, numerous publications have reported applications related to ultrasonic imaging and treatment. Second, due to their unique physicochemical properties such as having an electrostatically charged surface or allowing efficient surface adsorption, bulk NBs have also been widely used for flotation in mineral particle separation, waste-water treatment, oil separation, or surface cleaning. ${ }^{42-44}$ Third, the high pressure inside the bulk NBs allows extremely high gas solubility, which has proven to be beneficial for plants and animals in agriculture to supply oxygen and air NBs to water. ${ }^{9}$ The same NBs have proven beneficial for highly efficient combustion of fuels, resulting in energy savings and reduced generation of harmful gases. ${ }^{11,} 45$ More recently stimuli-responsive bulk NBs have also emerged, especially in connection with biomedical applications that will be described in more detail in Section 3.

\subsection{History of transient NBs}

Apart from long-living NBs, there exist transient NBs which have a short lifetime. Research on transient bubbles and their dynamics has a long history. Studies can be traced back to early research on cavitation in the first half of the last century. But since the 1970s, there have been numerous publications on the generation of transient bubbles using focused pulsed laser light, electrical discharge, high-powered ultrasound, or hydrodynamic cavitation, which occurs in a flowing liquid as a result of a decrease and subsequent increase in local pressure. However, most transient bubbles are generated in a size range of micrometers to even millimetres, ${ }^{46-48}$ probably at least in part because the characterization methods have a limited spatiotemporal resolution. Nevertheless it has been found that transient bubbles can also be formed at nanoscale sizes. Although there are very rare reports on transient NBs formed by pulsed lasers directly irradiating a liquid or solution, ${ }^{49}$ many authors have reported that transient NBs can be generated by a pulsed laser (typically in a pulse duration of nano-, pisco-, or femto-seconds) in combination with photothermal NPs such as gold NPs (AuNPs), or other photothermal nanomaterials such as titanium nanostructures or carbon based nanomaterials, or even organic molecules like hemoglobin. ${ }^{50,51}$ Recently, it has been reported that ultrasonication of electrolytes can also create transient NBs, which are generated from the fragmentation of larger bubbles near a glass surface by an acoustic field. ${ }^{52}$ The generation of transient bubbles can also occur as a result of a decrease and subsequent increase in the hydrostatic pressure that can be achieved by passing 
a liquid through a constricted zone at a suitable velocity. Reports on transient bubbles generated by the so-called "hydrodynamic cavitation" are traceable to approximately 10 years ago. Goedon et al. reported a flow-through apparatus designed as a chamber-inchamber device for nanocavitation, ${ }^{53,54}$ which further developed into an industrial application of neutralization of oil at the nanoscale (Nano Neutralisation ${ }^{\mathrm{TM}}$ ). ${ }^{55}$

Electrical discharges can also be used to generate transient NBs. Svetovoy et al. described transient hydrogen or oxygen NBs, with diameters smaller than $\sim 150 \mathrm{~nm}$ and a lifetime shorter than $150 \mu \mathrm{s}$, produced by water electrolysis using microsecond voltage pulses. ${ }^{33}$ Aogaki et al. reported that transient NBs could also be generated in aqueous electrolyte solutions by an electric field with the assistance of a magnetic field. ${ }^{56}$ In contrast to NBs produced by water electrolysis, the authors suggested that NBs are created by a magnetic field that induces the collision of ionic vacancies, which are initialized by an electrode reaction in the electric field.

\section{Stimuli-responsive NBs}

When directed at biomedical applications, both long-living and transient NBs have been developed that are generated by or respond to a specific stimulus to produce a certain action or signal. Stimuli can be external to the biological system to which the NBs are applied, such as the application of ultrasound, light, mechanical forces, temperature changes, electric fields, or magnetic fields. Alternatively, stimuli can be internal to the biological system of interest, such as a local change in $\mathrm{pH}$. In this review we will divide stimuli-responsive NBs into two classes: directly responsive NBs and indirectly responsive NBs. Directly responsive NBs are pre-existing (long-living) NBs whose size, shape, and other physicochemical properties can be altered due to an energy input from the stimulus. Indirectly-responsive NBs are instead formed by the stimulus itself, which first triggers the nucleation of NB and further stimulates NB growth and/or collapse in a liquid, subsequently inducing high local mechanical forces or chemical reactions. Below, we will overview both direct and indirect NB responses triggered by exogenous and endogenous stimuli.

\subsection{Ultrasound}

Ultrasound sound waves have frequencies higher than that of human hearing, ranging from $20 \mathrm{kHz}$ to several $\mathrm{GHz}$ and have been extensively studied and applied in stimuli-responsive microbubbles
(MBs). ${ }^{57}$ These MBs act as echo-enhancers since high acoustic impedance differences between gases and the surrounding medium enables them to resonate at frequencies used in clinical ultrasound imaging. When a bubble is stimulated by an ultrasound wave, the core gas can be expanded and compressed with the applied pressure's rarefaction and compression, respectively (Fig. 3).

At low acoustic power, corresponding to a low mechanical index (MI) (the $\mathrm{MI}$ is a unitless number that is defined as the peak rarefaction pressure of the ultrasound wave divided by the center frequency of the ultrasound wave), bubbles are symmetrically oscillating (Fig. 3a), which is also known as non-inertial cavitation. At intermediate acoustic power, bubbles present a uniform oscillation rate where expansion and contraction phases become unequal as the MBs resist compression more than expansion (Fig. 3b). This causes the return signal to contain multiple echoes called harmonics. In comparison, tissue has a relatively linear behavior due to its lower compressibility. This enables a unique opportunity to fine-tune signal processing and improve detection of the bubbles. At even higher acoustic power, the oscillation amplitude of the bubbles can grow rapidly during the low pressure phase, until the bubbles collapse due to the inertia of the inrushing fluid, inducing high local temperatures and mechanical forces generated by shockwaves or microjets (Fig. 3c). ${ }^{58,59}$ This process is also known as inertial cavitation.

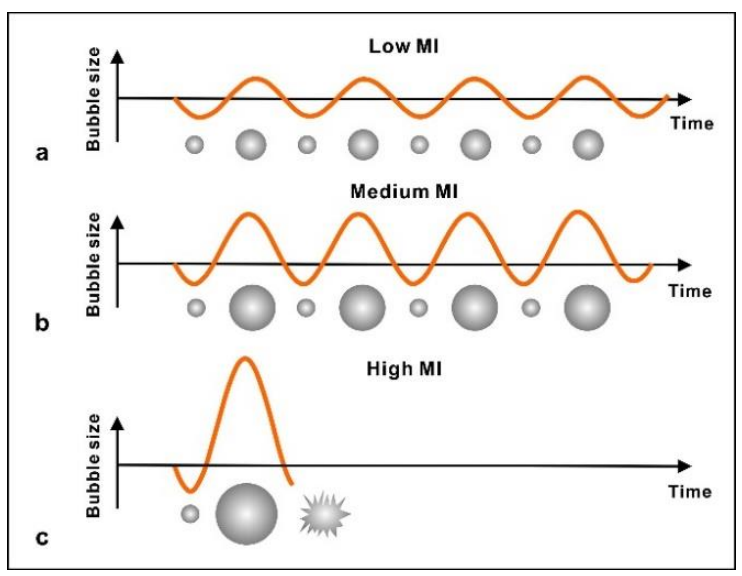

Fig. 3. MB dynamics under ultrasound stimulation. (a) MB linear oscillation with a low acoustic power for a low mechanical index (MI); (b) MB nonlinear oscillation with a medium acoustic power for a medium $\mathrm{Ml}$; (c) MB collapse under a high acoustic power for a high MI.

\subsubsection{Ultrasound-direct-responsive NBs}

Long-living NBs are well-known to respond to ultrasound stimuli. In order to maintain the bubble in a stable state for prolonged periods under ultrasound stimulation, the NB is structured as a core gas with 
a shell. A NB in solution (bulk NB) with a much smaller radius of curvature presents a very high internal (Laplace) pressure which will cause a rapid dissolution of the NBs. The presence of adsorbed material at the gas/water interface (the shell) can reduce or oppose the surface tension and thereby reduce or remove the driving force for dissolution and maintain the stability and long-life of the NBs. ${ }^{60,}$ 61 The shell typically includes surfactants, phospholipids, polymers, or proteins. ${ }^{62-67}$ In addition, polyethylene glycol (PEG) can be integrated to improve their stability, biocompatibility, and extend the circulation time in the body. ${ }^{68}$

Although many studies have reported numerous coating strategies that can be used to stabilize NBs under ultrasound stimulation, the mechanisms underlying ultrasound interaction with NBs are still under investigation. Recently, Hernandez et al. reported the effects of surface tension on the stability of NBs coated with phospholipids under ultrasound stimulation and found the shell composition had a substantial impact on membrane equilibrium surface tension. ${ }^{69}$ Results from this study show a significant decrease in the NB equilibrium surface tension through the incorporation of the surfactant Pluronic L10. In addition, the selection of the type of gas core in a NB is also critical to its stability. The use of a perfluorocarbon (PFC) gas core rather than air, nitrogen, or sulfur hexafluoride (all commonly used gasses in microbubble formulations) decreases bubble dissolution time due to its low solubility in fluids like blood. In Sections 4 and 5, we will introduce the techniques used for the generation of these coated NBs and their biomedical applications like biomedical imaging and drug delivery.

\subsubsection{Ultrasound-indirect-responsive NBs}

When ultrasound is used to irradiate bulk water solutions or hydrated tissue, tiny vapor/gas bubbles can emerge from pulsed ultrasound vibration. These types of indirect responsive MBs/NBs are preferentially formed at low frequencies and high intensities. ${ }^{59,70,71}$ A type of ultrasound-indirect-responsive NBs is one in which ultrasound stimulates the air filled gas core capped in nanopores in a mesoporous nanostructure (Fig. 4). ${ }^{72-75}$ One of the most interesting aspects in their biomedical application is that these NBs can be stimulated by ultrasound at a very low intensity and can undergo repeated nucleating cavitation reactions hundreds of times. As an example, Jin et al. showed bubbles generated from superhydrophobic mesoporous silica NPs with large surface areas which could be sustained for at least 30 min at a MI of 1.0, while lipid microbubbles only lasted for about $5 \mathrm{~min}$ at the same settings. ${ }^{76}$ Sviridov et al. demonstrated repeated ultrasound- stimulation of NBs from mesoporous silicon NPs leading to bubble collapse and energy release in the NP's vicinity. ${ }^{77}$

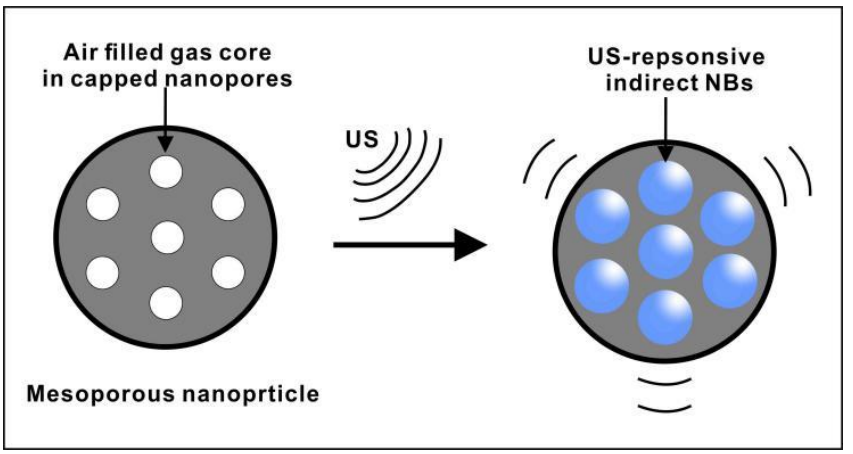

Fig. 4. Ultrasound-responsive indirect NBs generated by ultrasound which stimulate the gas trapped in the nanopores of a mesoporous silica nanoparticle.

Another approach of ultrasound-indirect-responsive NBs involves the ultrasound-stimulated phase-change of nanodroplets to microor nano-bubbles. To date, Perfluorocarbons (PFCs) used most commonly in NBs are dodecafluoropentane (DDFP) or perfluorohexane (PFH), which are liquids at room temperature. When coated with a lipid or polymer shell, nano-emulsions of DDFP and PFH are able to remain in solution at body temperature. When the droplet cores are heated by ultrasound energy and the temperature is higher than their boiling points (for DDFP $\left[\mathrm{C}_{5} \mathrm{~F}_{12}\right.$, boiling point $29^{\circ} \mathrm{C}$ ), they vaporize and form MBs/NBs. ${ }^{78}$ However, due to the Laplace pressure associated with the nanoscale size, quite high ultrasound energies are needed, which possibly leads to unwanted bioeffects. ${ }^{79-81}$ Although low-boiling-point PFC's have been proposed to lower the ultrasound threshold to about $4 \mathrm{MPa},{ }^{82}$ there remains a trade-off between lowering the acoustic vaporization threshold and colloidal stability of the NBs.

\subsubsection{Synthesis, characterization and biomedical applications of ultrasound-responsive NBs}

Ultrasound-direct-responsive NBs can be produced by both physical and chemical methods, although chemical approaches are mostly used for stabilization of shelled NBs. Examples of this include application of a lipid shell by hydration of a thin lipid film or coating with a polymer shell by emulsification. This is discussed in more detail in sections §4.2.1 Hydration of a thin lipid film and §4.2.2 Polymer emulsification. Characterization of NBs is possible by light microscopy techniques, electron microscopy or low resolution methods like dynamic light scattering (DLS), as is discussed in greater 
detail in sections $\$ 4.3 .1$ Light microscopy techniques, $§ 4.3 .2$ Techniques with high spatial resolution, §4.3.3 Techniques with low spatial resolution. When it comes to biomedical applications, ultrasound-responsive NBs have been used the most by far. They have been applied in bio-imaging including ultrasound imaging, molecular imaging, multimodal imaging (§5.1.1 Ultrasound imaging, §5.1.2 Molecular imaging, §5.1.4 Multimodal imaging), drug delivery including anticancer drug targeted delivery, nucleic acid and antibiotic delivery, delivery of therapeutic gases ( $\$ 5.2 .1$ Anticancer drug targeted delivery, §5.2.2 Nucleic acid delivery, §5.2.3 Antibiotic delivery, §5.2.4 Delivery of therapeutic gases) and ablation of tumor cells or biological tissues in section $\$ 5.3$ Ablation of tumor cells or biological tissues.

\subsection{Light}

Light is electromagnetic radiation. The electromagnetic spectrum of light used as stimulus of NBs usually derives from UV to near infrared wavelengths. The main light sources for optical stimulation of NBs are continuous-wave (CW) or pulsed lasers.

\subsubsection{Light-direct-responsive NBs}

When pre-existing NBs are illuminated with light, they can produce strong light scattering due to the refractive index difference at the interface between gas and liquid (Fig. 5). This property has been widely used to characterize long-living NBs or to detect the generation of transient NBs. Many publications have reported that long-living NBs can be detected by DLS to characterize their size. Recently, nanoparticle tracking analysis (NTA) was proposed to characterize the size and concentration of NB dispersions. NTA extracts this information based on images of the diffusional motion of individual NB which are visible through their scattered light. Further, light scattering by NBs (targeted to cancer cells) has been proposed to image cancer cells in vitro diagnostics. Bhandari et al. reported that single oxygen NBs in single cells can be precisely localized and tracked with dark field microscopy. ${ }^{83}$

\subsubsection{Indirect-responsive NBs stimulated by CW light}

CW light has been used to irradiate photothermal NPs such as metallic NPs dispersed in liquids to generate transient vapor NBs that emerge from the heated NPs. When the NPs are excited by a CW laser at the resonance frequency of surface plasmons (i.e., the collective oscillations of delocalized conduction electrons) with sufficient power, it can result in a rapid rise in temperature at the nanometer-scale in the vicinity of the particle surface, resulting in the formation of a vapor layer at the particle-liquid interface that can grow into so-called vapor NBs. Under continued illumination, the vapor volume increases and may potentially coalesce with other NBs, and eventually move to the liquid-air interface where the generated vapor is released and the NPs revert back to the solution to repeat the vaporization process. ${ }^{34,84,85}$

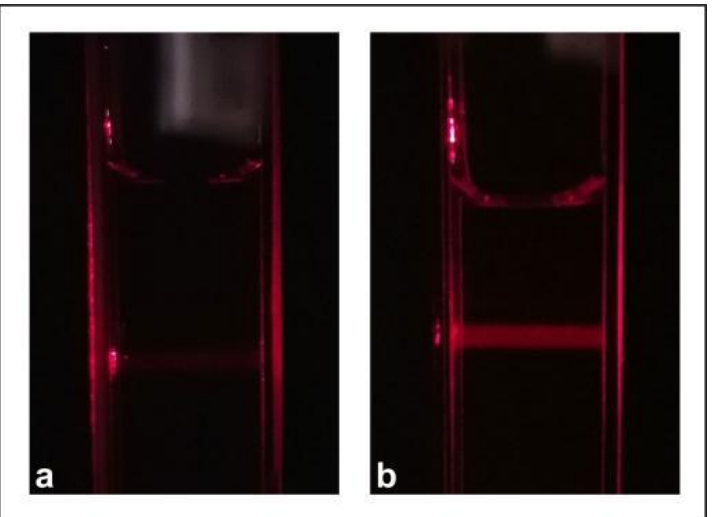

Fig. 5. A laser beam passing through a cuvette (a) without and (b) with NBs, showing enhanced scattering when NBs are present. The figure is adapted from reference ${ }^{86}$.

Recently, numerous studies have reported on the underlying process of vapor NB formation from NP heated with a CW laser. Hou et al., for instance, did this for AuNPs. ${ }^{87}$ Based on (time-resolved) acoustic measurements the authors found a clear echo signature could be observed upon the formation of vapor NBs. To better understand vapor NB generation, Fang et al. quantified the precise properties of the liquid-vapor phase transition at the NP surface, such as temperature, internal pressure, and size of the vapor NB formed upon resonant $\mathrm{CW}$ laser excitation of AuNP. They did this by combining dark-field scattering measurements of localized surface plasmon resonance (LSPR) shifts with surface-enhanced Raman scattering (SERS)-based temperature measurements. ${ }^{84}$ The authors demonstrated that the temperature on the AuNP surface showed a dramatic drop due to the formation of a nanoscale vapor layer which thermally decouples the NP from the surrounding liquid. Conversely, using molecular dynamics simulations, Lohse et al. demonstrated that dissolved gas was one of the conditions required for nucleation of a NB and investigated its impact on the NP growth dynamics. ${ }^{88}$ The authors found that dissolved gas above a certain threshold concentration could dramatically facilitate vapor NB nucleation due to the formation of gaseous weak spots in the surrounding liquid.

\subsubsection{Indirect-responsive NBs stimulated by pulsed light}


When using intense short laser pulses (typically less than 10 nanoseconds), very high temperatures of NPs can be achieved before heat can diffuse from the NP into the environment. Thus, the NPs temperature can rapidly increase to several hundred or even thousand degrees in less than a nanosecond, leading to the almost instantaneous evaporation of the liquid layer surrounding the NPs and resulting in the formation of transient vapor NBs. The vapor NBs first expand and then collapse after having reached their maximal size, causing a strong local mechanical effects like high-pressure shockwaves or liquid jets (left panel in Fig. 6). ${ }^{50,89,90}$

The threshold fluence of a laser pulse to generate NBs is mainly determined by the pulse duration and the characteristics of the NPs including their size and shape. The laser fluence threshold for NB has been discussed in several published reports experimentally and quantitatively. ${ }^{91-93}$ The most interesting finding was that the laser fluence threshold is a function of the gold nanosphere's diameter. Metwally et al. used numerical simulations to study the physics of bubble nucleation around gold nanospheres to provide a possible explanation of the above findings. ${ }^{94}$ They found that the minimum threshold for NB generation is achieved for gold nanospheres with a diameter of approximately $60 \mathrm{~nm}$. They further explained that higher fluences are needed for smaller NPs due to rapid energy dissipation to the surroundings (due to the high surface to volume ratio), and the required fluence is also larger for larger NPs $(>60 \mathrm{~nm})$ due to the nonlinearity of the absorption cross-section with particle size. Lapotko et al. explored 10 nanosecond, 500 picosecond, or 70 picosecond pulsed laser light to irradiate gold nanospheres, gold nanorods, or gold nanoshells. ${ }^{95-97}$ They found that the threshold for the formation of NBs significantly depended on the laser pulse duration and the type of NPs used. The laser intensity threshold of a 10-nanosecond pulsed laser light was more than 10-fold higher than for 500 picosecond pulses. This was due to the fact that thermal equilibration and the onset of heat diffusion in the environment occurred at the 100 picosecond timepoint. A large part of nanosecond laser pulses, therefore, do not contribute to the sudden rise in temperature that is needed for NB formation. The authors also found that gold nano-shells often required much lower laser energy than spherical AuNPs. Recently, Fales et al. further confirmed the above findings and found that the threshold laser intensity for nanoshells was the lowest and also pointed out that silica coated gold nanorods could significantly reduce the NB formation threshold. ${ }^{56}$ By incorporating low boiling point of PFC into the hollow gold nano-shell interior cavity, the threshold laser intensity for the generation of NBs can be further reduced by more than half. 98,99

Recently, some studies have also reported on the dynamic response of pulsed laser induced vapor NB around photothermal NPs. Lombard et al. found that the temporal evolution of the NB is asymmetrical with adiabatic expansion, while the collapse is an isothermal evolution. ${ }^{100} \mathrm{~A}$ recent study reported that the NB dynamic collapse occurs via two different mechanisms: a rapid partial bubble collapse governed by vapor condensation or a slow diffusioncontrolled bubble dissolution. ${ }^{101}$ The authors found that a higher laser fluence could lead to the generation of NBs containing more water vapor and whose collapse is dominated by vapor condensation. Instead, longer pulses and more dissolved air concentrations in the liquid can generate bubbles containing more gas whose collapse is more greatly affected by diffusion-controlled dissolution. Nonetheless, these studies focused on NB dynamics around single individual NPs. Nakajima et al. showed that NP concentration had an effect on NB dynamics stimulated by a pulsed laser. ${ }^{102}$ Indeed, the pressure waves arising from NPs, even when the inter-particle distance among NPs is as far as $30 \mu \mathrm{m}$, can still influence NB formation from neighboring NPs when irradiated at a high laser fluence.

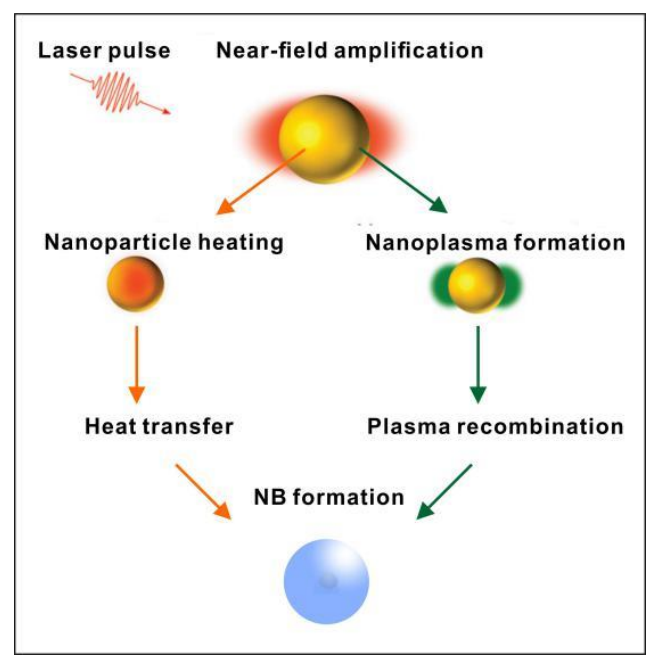

Fig. 6. Light-responsive NB formation by heat transfer or plasma recombination under pulsed laser irradiation. The figure is adapted from reference ${ }^{103}$.

When optical stimulation is achieved using a femtosecond laser pulse, localized surface plasmon resonance could also induce a near field enhancement of the NP. In these regions, plasma recombination may occur from multiphoton ionization of the medium. The plasma cools down by collision and recombination with water molecules, 
hence producing a quick temperature rise and pressure increase of the water, which leads to the generation of a water vapor NB around the irradiated NP (right panel in Fig. 6). The use of plasma-mediated NBs was first reported by Meunier et al., who performed femtosecond-pulsed laser (45 fs) irradiation of $100 \mathrm{~nm}$ gold nanospheres. ${ }^{104-106}$ Under these conditions they found that the threshold of plasma-mediated NB formation is similar as thermal induced NBs. Recently, the same group reported a multiscale modeling computational approach to describe the generation and dynamics of NBs induced using both heat transfer and plasmon deexcitation mechanisms. ${ }^{103}$ Based on time-resolved imaging and spectroscopy data, they demonstrated that the bubble size, dynamics, and formation threshold can be quantitatively predicted with acceptable error.

Of note, indirect-responsive NB can also be generated from a liquid without photothermal NPs when strong laser pulses are used. When using nanoseconds or picosecond laser pulses of sufficient fluence, they can induce optical breakdown in the liquid resulting in bubble formation, due to heating and subsequent local evaporation of the water, and expansion. However, the size of these bubbles ranges typically from tens to hundreds of micrometers. ${ }^{107,108}$

Such bubbles can also be formed by femtosecond laser pulses which induce plasma formation following a multi-photon absorption process. Free electrons in the plasma thermalize within tens of picoseconds. As this is much shorter than the characteristic time for acoustic wave propagation out of the focal volume, the thermoelastic stress caused by the temperature rise will be confined to the focal volume. Subsequent propagation of the pressure wave causes substantial tensile stress in the center of the focal volume. If the tensile strength of the water is exceeded, a nucleation bubble will be formed. ${ }^{49}$ Vogel et al. measured the maximal bubble size growth caused by a femtosecond pulse laser at 347, 520, and 1040 $\mathrm{nm}$ irradiation in water and found the maximal radius ranged from $190 \mathrm{~nm}$ to $320 \mathrm{~nm}$, which is much smaller than those generated by pico- or nanosecond laser pulses. ${ }^{109}$

\subsubsection{Synthesis, characterizations and biomedical applications of light-responsive NBs}

Light-direct-responsiveness is an intrinsic feature of any NB. Therefore, all synthesis methods reported sections §4.1 Physical methods, $\S 4.2$ Chemical formulations are of relevance in this regard.
Characterization of light-direct-responsive NBs is mostly performed by light microscopy, electron microscopy or DLS (§4.3.1 Light microscopy techniques, §4.3.2 Techniques with high spatial resolution, §4.3.3 Techniques with low spatial resolution). However, characterization of light-indirect responsive-NBs is mostly done by low spatial resolution methods like light scattering for which their short lifetime is less of an issue (see details in section §4.3.3 Techniques with low spatial resolution). Both light-direct- and indirect-responsive NBs have been widely used in biomedical applications, such as bio-imaging for diagnosis of diseases, drug delivery, or tissue ablation for therapy. This is further elaborated in sections $\S 5.1$ Biomedical applications, $§ 5.2$ Drug delivery, $§ 5.3$ Ablation of tumor cells or biological tissues).

\subsection{Mechanical forces}

Herein, mechanical forces are referred to as the application of a load, shock waves, or hydrodynamic pressure.

\subsubsection{Mechanical force-direct-responsive NBs stimulated by a load}

Several studies have reported responses induced by surface NBs when stimulated by applying a load with an AFM tip. It was observed that the NB gas-liquid (water) interface behaved like a Hook spring with a stiffness between 60 and $120 \mathrm{pN} / \mathrm{nm}$, close to the surface tension of the air-water interface $(\sim 72 \mathrm{pN} / \mathrm{nm}) .{ }^{110-112}$ Larger NBs were slightly softer than the smaller ones possibly due to a smaller Laplace pressure.

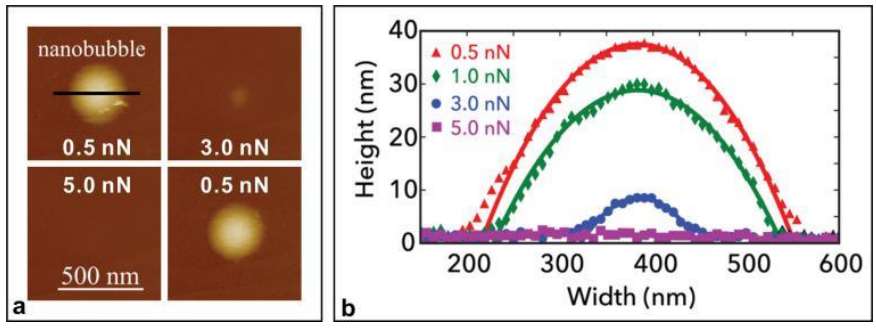

Fig. 7. AFM height images of a nanobubble in Peak Force (Fp) mode. (a) Successive AFM images captured for peak forces $\mathrm{Fp}=0.5,3.0,5.0 \mathrm{nN}$. A final scan was taken at $\mathrm{Fp}=0.5$ $\mathrm{nN}$, showing that the objects were not destroyed by the scanning. (b) Cross-sectional profiles of the nanobubble as indicated top left panel (a). The figures are adapted from reference $^{113}$.

Through a combination of a tapping, lift, and force volume mode AFM study, Walczyk et al. found that the strength and the magnitude of the bubble deformation were not the same over the entire surface of the NBs, but rather depended on the position of the tip on the bubble surface, and also determined that the effective surface 
tension of the NB was not uniform. ${ }^{114}$ They also reported that NBs deformation occurred more severely with hydrophobic AFM tips than with hydrophilic tips. Recently, using AFM in peak force mode, An et al. found NBs were completely pushed against the surface which resulted in a complete disappearance of the height profiles at $\mathrm{Fp}=5.0 \mathrm{nN}$, even though they were not physically moved or destroyed (Fig. 7). ${ }^{113}$

\subsubsection{Mechanical force-direct-responsive NBs stimulated by shock waves}

A shock wave-induced collapse of a pre-existing gas or vapor NB in water was reported by several publications mostly by using molecular dynamics simulations. Vedadi et al. observed a focused jet at the onset of bubble shrinkage and a secondary shock wave upon bubble collapse. ${ }^{115}$ The jet length scaled linearly with the NB radius, as observed in experiments with micro- to millimeter sized bubbles. Subsequently, the same group reported a shock wave-induced collapse of NBs near an amorphous silica surface and found the surface to be pitted by the water jet, which produced pits of similar size as the bubble. ${ }^{116}$ The authors further pointed out that gas filled NBs did not collapse completely and induced much less damage to the silica surface as the nanojets were much weaker than those resulting from the collapse of empty NBs.
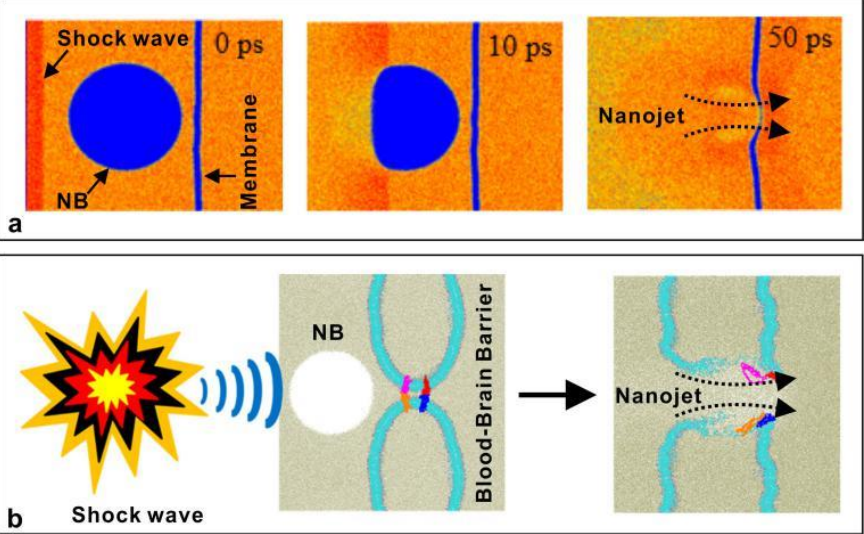

Fig. 8. (a) Shock wave induced NB collapse interacting with a cell membrane. Twodimensional water density maps showing the collapse of a NB after the shock wave passage. (b) Shock wave induced NB collapse interacting with a blood-brain barrier tight junction. The figures are adapted from references. ${ }^{117,} 118$

Berkowitz et al. performed molecular dynamics simulations of cell membrane poration following shock wave-induced NB collapse. ${ }^{117}$ They found that the shock wave first hit the cell membrane, which was followed by the generation of a nanojet leading to the poration of the membrane (Fig. 8a). However, in the absence of the NB, a shock wave alone, even with an impulse as high as $18 \mathrm{mPa}$, could not create a pore in the membrane. The same group also reported that based on their simulations, a shock wave-induced NB collapse could also open a blood-brain barrier tight junction (Fig. 8b). ${ }^{118}$

Recently, Zhang and Joshi extended these studies to multiple NBs They found that the collapse of multiple NBs not only led to the creation of larger pores, but also increased the pores density on the cell membrane. ${ }^{119}$

\subsubsection{Mechanical force-indirect-responsive NBs stimulated by} hydrodynamic pressure

The variation of hydrodynamic pressure through a constriction channel (i.e., a Venturi orifice) can also stimulate the generation of cavitation bubbles and is typically known as hydrodynamic cavitation. ${ }^{120}$ When hydrodynamic cavitation is nucleated in the channel, the response of the bubble can be inertial or non-inertial cavitation upon stimulation by ultrasound.

\subsubsection{Synthesis, characterizations and biomedical applications} of mechanical force-responsive NBs

The mechanical force-direct-responsive NBs are mostly fabricated by physical methods like the straight-forward solvent exchange methods (see details in section §4.1.6 Solvent exchange). Most of the methods reported in section 4 are well-suited to characterize this type of responsive NBs. Mechanical force-responsive NBs have been used in biomedicine, for instance for the ablation of biological tissue. One example is that of indirect-responsive NBs which are induced by the hydrodynamic pressure that is applied for destroying kidney stones (§5.3 Ablation of tumor cells or biological tissues).

\subsection{Temperature changes}

NBs have been reported to respond to temperature changes of the bulk solution liquid of the NBs dispersion.

\subsubsection{Temperature-responsive NBs stimulated by temperature changes above the freezing point}

Several studies have described the influence of temperature on surface NBs. When the bulk water temperature increased from 20 to $50^{\circ} \mathrm{C}$, there was a significant increase in the surface density of NBs in the temperature range of 30 to $35^{\circ} \mathrm{C}$. The bubble size was found to be maximal between $35-40^{\circ} \mathrm{C} .{ }^{121}, 122$ This is probably due to the minimal solubility of air in water at this temperature range resulting in the largest saturation level. ${ }^{123}$ When the bulk water temperature 
returned to ambient conditions $\left(20-25^{\circ} \mathrm{C}\right)$, the formed NBs retained their structure.

A similar result was also observed for bulk NBs. Ahmadi and Darban reported that the size of NBs created by hydrodynamic pressure in Venturi tubes increased from $200 \mathrm{~nm}$ to approximately $350 \mathrm{~nm}$, and was associated with a temperature increase from 20 to $40^{\circ} \mathrm{C} .124$

\subsubsection{Temperature-responsive NBs stimulated by changes in temperature below the freezing point}

While most publications have evaluated temperature changes above the freezing point, recently Nirmalkar et al. observed that bulk NBs in a pure water suspension, which were produced by an acoustic cavitation technique at room temperature of $20^{\circ} \mathrm{C}$, disappeared after freezing to $-18^{\circ} \mathrm{C}$ and thawing of the suspension (Fig. 9). ${ }^{125} \mathrm{~A}$ possible explanation is that NBs are forced to move and coalesce or agglomerate in the growing presence of ice crystals, leading eventually to the rupture of the NB interface. Indeed, when a sufficient amount of surface-active agent is added to the NB suspensions in pure water before freezing, the NBs were shielded against the effects of freezing and thawing and could prevent the collapse of NBs, which are entirely preserved by the surface-active agent. As this property of NBs disappearing after freezing and thawing of the suspension is considered an intrinsic feature of NBs, it has also been applied to distinguish NBs from contaminants such as solid NPs or nanodroplets. ${ }^{126}$
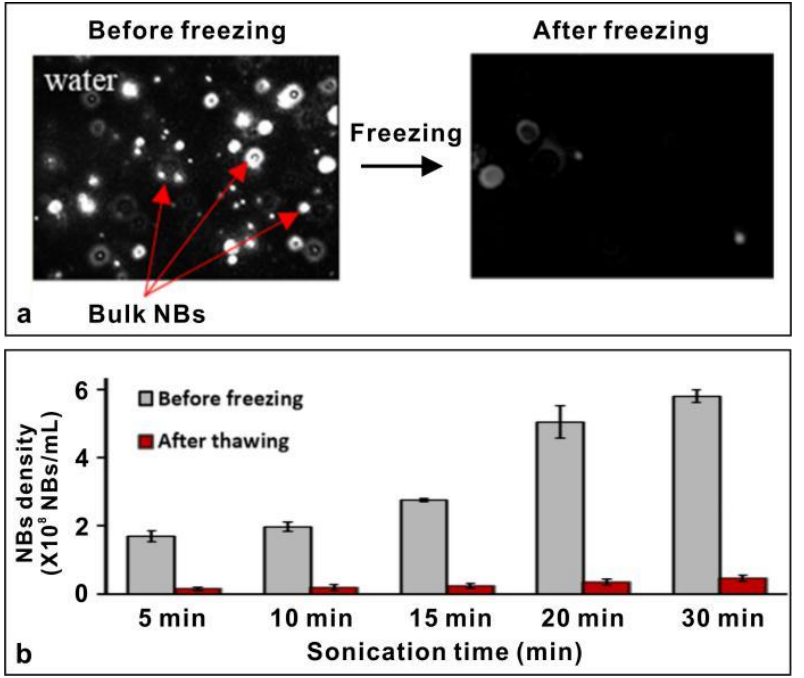

Fig. 9. Response of NBs by temperature changes below the freezing point. (a) A typical image showing the freezing and thawing of bulk NBs and (b) the quantified concentration of NBs suspensions generated in pure water at different sonication times. The figures are adapted from reference. ${ }^{125}$
3.4.3

Synthesis, characterizations and biomedical applications of temperature-responsive NBs

The temperature-responsive NBs are typically made by physical methods (see section §4.1 Physical methods ). Typically, light microscopy techniques and DLS are used to characterized to these NBs (see sections §4.3.1 Light microscopy techniques, §4.3.3 Techniques with low spatial resolution). Temperature-responsive NBs have not been used in biomedicine so far.

\subsection{Electric fields}

Electric field-responsiveness of NBs is another intrinsic feature of long-living NBs that often have a certain surface charge.

\subsubsection{Electric field-direct-responsive NBs}

Long-living NBs often have a negative charge, although their surface charge is determined by the $\mathrm{pH}$ of the solution, salt concentrations in the solution, or by their surface coating. Surface charges cause bulk NBs to move in the liquid in response to an electric field, for instance allowing to determine the NB's zeta potential.
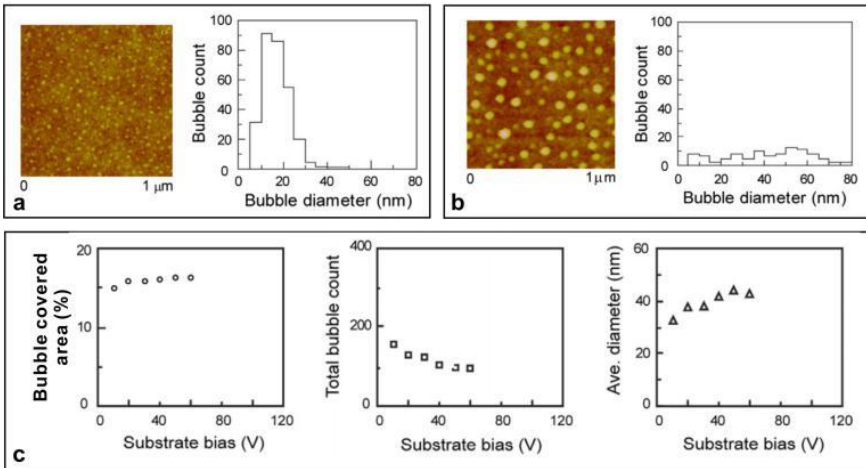

Fig. 10. Response of surface NBs to an external electric field in terms of size and concentration. AFM height images and corresponding histograms of the size distribution of surface NBs on a polystyrene surface as a function of an applied electric field of (a) 0 $\mathrm{V}$ and (b) $40 \mathrm{~V}$ in distilled (DI) water; and (c) geometrical distribution of NBs as a function of the applied electric field on the PS surface in DI water. The figures are adapted from reference. ${ }^{127}$

Conversely, external electric fields could also have an effect on surface NBs. For instance, Bhushan et al. characterized the electric field effect $(0-60 \mathrm{~V})$ on the responses of surface NBs generated on hydrophobic polystyrene (PS) films immersed in distilled water using AFM. ${ }^{127}$ As the positive voltage increased, the average diameter of the resultant NBs increased while the total count of the NBs decreased. The possible reason is that the applied electric field pulls the negatively charged surface NBs towards the surface and results in aggregation leading to an increase in the size of NBs and a decrease 
in the total count, despite the almost constant total area covered by NBs (Fig. 10).

In contrast, Wu et al. recently simulated the effects of an external electric field on carbon dioxide $\left(\mathrm{CO}_{2}\right) \mathrm{NBs}$ at the surface of hydrophobic particles using molecular dynamics simulations and found that the external electric field can reduce the size of NBs. ${ }^{128}$ They proposed it was possible because the active gas molecules stimulated by the external electric field can escape from the bubbles to the water, which results in a decrease in size. While this seems to contradict the experimental findings by Bhushan et al., the studies are based on different experimental conditions. For example, the size of NBs are tens of nanometers in diameter in the Bhushan et al. experiments, which are more than ten-fold larger than the NBs simulated by Wu et al. In addition, Wu et al. only simulated single NBs and did not consider interactions of NBs with each other.

\subsubsection{Electric field-indirect-responsive NBs stimulated by} electrolysis

Under an electric field, a large quantity of gas molecules can be generated in electrochemical reactions and produce either surface or bulk NBs through electrolysis due to the substantial gas supersaturation near the electrode's surface.

Zhang et al. reported the earliest direct experimental observation of surface NBs produced by electrochemical reactions using a combination of an electrochemical reaction cell and in situ AFM in the tapping mode. ${ }^{129}$ The study showed that the formation and growth of surface NBs on highly oriented pyrolytic graphite (HOPG) could be controlled by the applied DC voltage $(-1.4$ to $-2 \mathrm{~V})$. A greater number and larger bubbles formed after a longer reaction time for a given applied potential and likewise under a higher potential for a given reaction time. In order to overcome the larger surface area of the working electrode and induce the distribution of a highly nonuniform electric field over the HOPG surface, which would adversely affect the control of gas saturation and thus eventual NBs nucleation, nanoscale electrodes were developed to generate single NBs of hydrogen $\left(\mathrm{H}_{2}\right)$, oxygen, and nitrogen of radii ranging from 2.5 to $90 \mathrm{~nm}$ on nanoelectrodes. ${ }^{129-133}$

However, these experiments lacked the spatial resolution to explain the process of nucleation and formation of NBs and their shape. Recently, Sirkin et al. studied the process of nucleation of NBs on nanoelectrodes using molecular simulations. ${ }^{134}$ The results confirmed that the surface or bulk NBs nucleate through the classical mechanism of gas molecule supersaturation (Fig. 11a). Competition between binding of water and gas to the electrode determined whether it resulted in a bulk NB or surface NB (Fig. 11b).
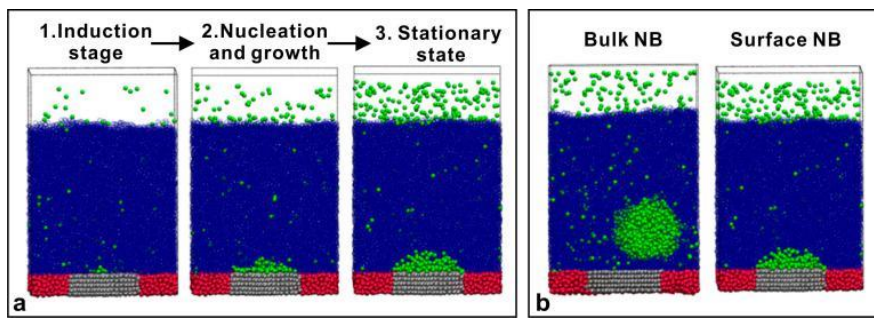

Fig. 11. (a) Snapshots of three different stages of formation of a surface NB: induction nucleation and growth, and the stationary state. (b) A solution nucleates homogeneously close to the electrode and does not bind to the surface or block the electrochemical reaction (left panel); NBs nucleated at the electrode in heterogeneous remains attached to the electrode, blocking the electrochemical reaction (right panel). The figures are adapted from the reference ${ }^{134}$

Ghaani et al. recently reported a method for massive generation of metastable bulk NBs in water using static electric fields ( 12 kV/m), which has great potential for applications in biomedicine for the production of oxygen NBs for oxygen delivery. ${ }^{135}$ They claimed that: 1) this method has a much lower energy expenditure of as low as 0.3 $\mathrm{W} \mathrm{h} / \mathrm{m}^{3}$ in comparison with other available advanced systems requiring typically $\sim 40 \mathrm{~W} \mathrm{~h} / \mathrm{m}^{3}$; and 2) much a higher throughput of $\mathrm{NBs}$ is generated achieving $25-35 \mathrm{mg} / \mathrm{L}$ of dissolved oxygen at standard temperature and pressure, which is much higher compared with typical levels of no more than $2 \mathrm{mg} / \mathrm{L}$.

The above studies focused on the use of a constant DC electric field to trigger NB nucleation and growth. Instead, DC or AC electric fields with frequencies between 20 and $100 \mathrm{kHz}$ can also be used for electrolysis and thus are able to electrochemically generate transient NBs with $\mathrm{H}_{2}$, oxygen, or their mixture, to densely cover the electrodes. ${ }^{136-139}$ It has also been shown that the resulting violent implosions of NBs/MBs can damage platinum electrodes.

\subsubsection{Synthesis, characterization and biomedical applications of electric field-responsive NBs}

Since electric field-responsiveness of NBs is an intrinsic feature of long-living NBs, all of the synthesis method reported in section 4 are suited to fabricated this responsive NBs. Also all of the characterization methods reported in section 4 are suitable to characterize these responsive NBs. This responsiveness to electric fields has been widely used to characterize NBs in terms of size or zeta potential, which are two of the most important features of NBs 
designed for biomedical applications (see section $\S 5$. Biomedical applications). Conversely, indirect-responsive NBs generated by electric fields are reported to be a promising method for high throughput generation of NBs that can be used in biomedical applications (see section §5. Biomedical applications).

\subsection{Magnetic fields}

Typically, pre-existing gaseous NBs are required to be further modified to become responsive to a magnetic-field. Conversely, indirect-responsive NBs generated by a magnetic field have also been produced.

\subsubsection{Magnetic field-direct-responsive NBs}

In order to render pre-existing NBs responsive to magnetic fields, they normally require coating with magnetic materials like superparamagnetic iron oxide or gadolinium, which can be embedded in the NB shells. ${ }^{140-142}$
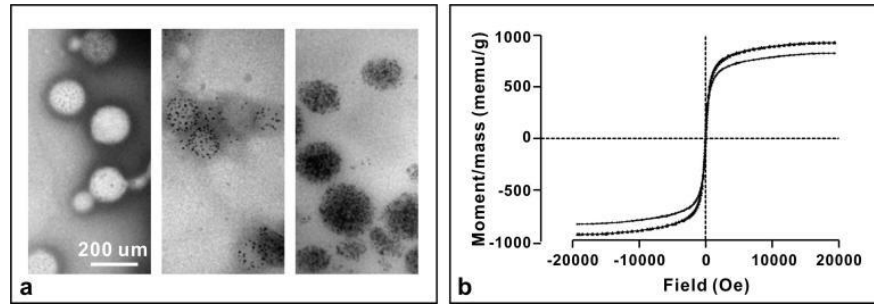

Fig. 12. (a) Transmission electron microscope images of NBs prepared with differen amounts of ultrasmall superparamagnetic iron oxide $(0,0.4$, and $1.6 \mathrm{mg})$ from left to right. (b) The magnetic properties of paclitaxel-ultrasmall superparamagnetic iron oxideembedded NBs. The figures are adapted from reference ${ }^{141}$

NBs modified with such magnetic elements can be moved and guided to a specific location by an external magnetic field, e.g. for drug delivery. For example, Huang et al. guided NBs coated with superparamagnetic iron oxide NPs embedded in a silica shell to a specific brain area of mice by applying a magnetic field in vivo. The local accumulation of NBs in the vasculature caused a targeted disruption of the blood-brain barrier and achieved drug delivery in the brain. ${ }^{140}$ Conversely, NBs modified to be magnetic-responsive can also be used as contrast agents for MRI. Song et al. described ultra-small superparamagnetic iron oxide embedded NBs (Fig. 12a); a lack of hysteresis confirmed their superparamagnetic nature (Fig. 12b). ${ }^{141}$ These results showed that NBs with magnetic-responsive properties had a coercivity value of 7.48 Oe, which suggested that these magnetic NBs exhibited superparamagnetic and a monodomain behavior, indicating that, when the magnetic field is removed, they will exhibit nonpermanent magnetization for $\mathrm{T} 2$ - weighted MRI. Finally, as NBs are intrinsically responsive to ultrasound, the magnetic-responsive NBs can be used as multimodal imaging contrast agents. ${ }^{142}$

\subsubsection{Magnetic field-indirect-responsive NBs}

While the formation of NBs by the application of a magnetic field alone has not been reported so far, they can be formed in a combined electro-magnetic field, as induced by a solenoid coil. ${ }^{143,} 144$ Although the exact mechanism involved in the nucleation of NBs under electromagnetic fields is not well known, Vallee et al. proposed that the external electromagnetic field can enhance the adsorption of ions at the gas-liquid interface and can induce the generation of a shell of counterions that further induce the formation of NBs and improve their stability. ${ }^{143}$

\subsubsection{Synthesis, characterization and biomedical applications} of magnetic field-responsive NBs

Magnetic field-direct-responsive NBs are typically fabricated by chemical means because magnetic materials like superparamagnetic iron oxide or gadolinium should be incorporated into the NB's shell (see section $\$ 4.2$ Chemical formulations). This type of NBs are often characterized by electron microscopy to visualize the embedded magnetic materials in the shell or by DLS to measure their size (see section \$4.3.2 Techniques with high spatial resolution). Such magnetic field-responsive NBs have been reported to be useful for biomedical applications including magnetic resonance imaging (MRI) imaging or magnetic-guided drug delivery (see details in sections $\S 5.1 .4$ Multimodal imaging, $§ 5.2 .4$ Delivery of therapeutic gases).

\section{7 $\mathrm{pH}$ changes}

The response of a NB to a change in $\mathrm{pH}$ is an intrinsic feature, as will be explained in this section.

\subsection{1 pH-direct-responsive NBs}

Uncoated $\mathrm{MBs} / \mathrm{NBs}$ in aqueous media typically have negative charges on their surfaces, because the cations are more likely hydrated and thereby have a tendency to stay in the aqueous medium, while the smaller less hydrated and more polarized anions trend to be adsorbed on the bubble surface. ${ }^{145-148}$ The zeta potential of uncoated NBs in an aqueous solution is greatly affected by the $\mathrm{pH}$ of water and tends to decrease (more negative) with increasing $\mathrm{pH}$ values (Fig. 13). Considering the study by Calgaroto et al. as an example (square dots in Fig. 13a), the zeta potential of gaseous NBs 
is about $-10.0 \mathrm{mV}$ at $\mathrm{pH}=6.5$. At such a $\mathrm{pH}$ value, the concentration of the $\mathrm{OH}^{-}$ion is equal to that of $\mathrm{H}^{+}$. However, the positive ions $\left(\mathrm{H}^{+}\right)$ preferentially remain in the bulk aqueous phase leaving space at the gas-liquid interface of the NB for negative ions, a phenomenon called "negative adsorption" or "proton exclusion". ${ }^{149}$ When the $\mathrm{pH}$ value is decreased, the concentration of $\mathrm{OH}^{-}$exponentially decreases, while the $\mathrm{H}^{+}$concentration greatly significantly increases, resulting in a decrease of the negative charge density at the NB surface, which may even reverse the net NB surface charge. Thus, as a result, the zeta potential of NBs exhibits a positive value in very strong acid solutions. The opposite happens when the $\mathrm{pH}$ of the solution increases above 7, leading to highly negatively charged NBs.

However, when a solution contains surfactant molecules such as the anionic surfactant sodium dodecyl sulphate (SDS), the tendency of the NB's zeta potential to decrease with increasing $\mathrm{pH}$ becomes much less significant (Fig. 13b). The reason is probably that SDS surfactants with their negative hydrophilic head become adsorbed through electrostatic forces with the polar head towards the bubble (which has a positive charge at low $\mathrm{pH}$ ), causing a change in the NB's zeta potential. 44
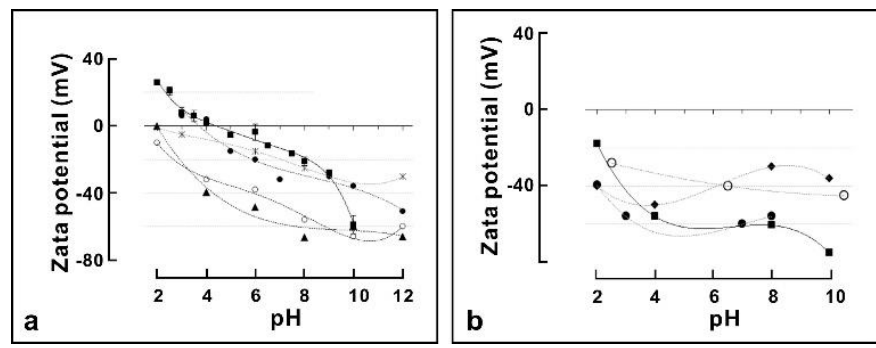

Fig. 13. (a) Zeta potentials of $\mathrm{MBs} / \mathrm{NBs}$ as a function of $\mathrm{pH}$. (b) Zeta potential of bubbles as a function of $\mathrm{pH}$ in the presence of sodium dodecyl sulphate (SDS). The different symbols show results from different independent studies. The figures are adapted from reference $^{44}$.

Some studies have reported on the influence of $\mathrm{pH}$ on the size of NBs. Calgaroto et al. determined that the size of long-living bulk NBs increased with $\mathrm{pH}$, growing from an initial size of $150 \mathrm{~nm}$ at a $\mathrm{pH}$ of 2 to a maximum of $720 \mathrm{~nm}$ at a $\mathrm{pH}$ of approximately 4.5 , where NBs are almost uncharged $( \pm 5 \mathrm{mV})$. Their study showed that the higher charge of the NBs, the smaller their size, especially at low $\mathrm{pH}$ values. ${ }^{44}$ However, Cho et al. observed that the size of NBs did not depend on the $\mathrm{pH} .{ }^{150}$ Nirmalkar et al. found that above $\mathrm{pH} 4$, the bubble size is more or less unaffected by $\mathrm{pH}$ changes, but when $\mathrm{pH}$ is below 4 , the bubble diameter increases significantly as the $\mathrm{pH}$ decreases further. ${ }^{126}$ The reason for these seemingly contradicting results is probably because different methods of NB formation were used: depressurization of saturated air water, ultrasonication, and hydrodynamic cavitation.

The $\mathrm{pH}$-dependent response of NBs can be modified depending on the coating that is applied. It has been reported that $\mathrm{pH}$-responsive NBs are made by coating with acetalated dextran with acetal moieties, which consist of a $\mathrm{pH}$-sensitive polymer that respond to minor changes in $\mathrm{pH}$ (Fig. 14). ${ }^{151-153}$ Its $\mathrm{pH}$-responsive property enables the release of oxygen to overcome the hypoxic conditions in the tumor microenvironment, by taking advantage of the slight difference in $\mathrm{pH}$ between healthy tissues $(\sim 7.4)$ and the acidic extracellular environment of solid tumors (6.0-6.5).
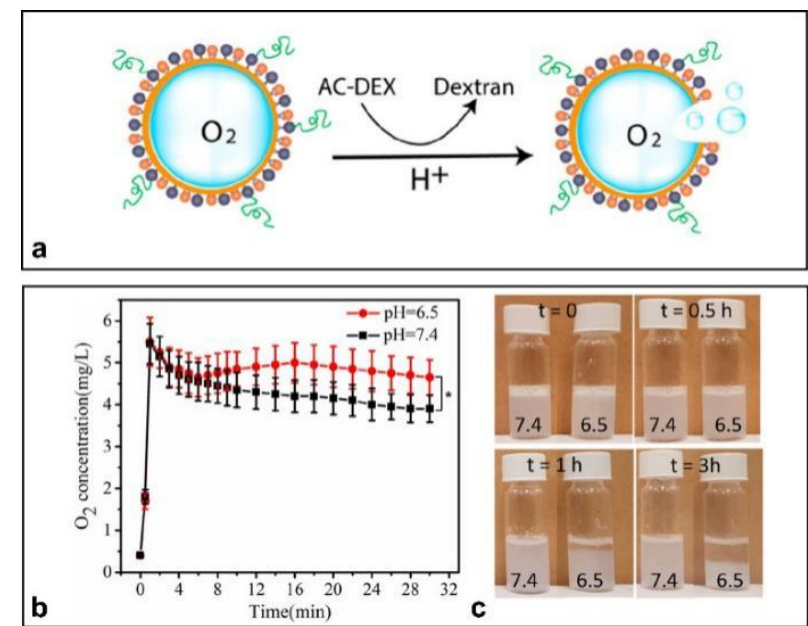

Fig. 14. (a) Oxygen NBs enclosed by the acetalated dextran polymer shells in response to a drop in $\mathrm{pH}$. (b) In vitro oxygen release kinetics of the oxygen NBs incubated at $\mathrm{pH} 6.5$ and 7.4. $\left({ }^{*} \mathrm{p}<0.05\right)$. (c) Optical images of the oxygen NBs stored in a neutral solution (pH 7.4) and an acidic solution ( $\mathrm{pH} 6.5$ ) for $3 \mathrm{~h}$. The figures are adapted from reference ${ }^{153}$.

Conversely, Li et al. described NBs coated with a shell composed of a mixture of poly(lactic-co-glycolic acid) (PLGA) and oleylamine. ${ }^{142}$ When the $\mathrm{pH}$ of the environment changed from 7.4 to 5.0, the zeta potential of NBs changed from -11.6 mV (pH 7.4) to $6.2 \mathrm{mV}(\mathrm{pH} 6.4)$ and $13.7 \mathrm{mV}$ ( $\mathrm{pH}$ 5.0). These results indicated that the NBs presented $\mathrm{pH}$-dependent charge-switchable behavior. The authors further presented the cumulative release of the drug fluorouracil (5-FU) from the NBs, which reached 9.3\% (pH 7.4), 27.3\% (pH 6.5), and $42.3 \%(\mathrm{pH} 5.0)$ in $48 \mathrm{~h}$. The NBs having a $\mathrm{pH}$-dependent drug-release capacity presented greater 5-FU release at lower $\mathrm{pH}$ values, probably because the surface of the NBs underwent greater protonation at more acidic $\mathrm{pH}$ values, which in turn triggered greater 5-FU release in the acidic environment.

\subsection{2 $\quad \mathrm{pH}$-indirect-responsive NBs}


$\mathrm{pH}$ changes can also be used to generate NBs in combination with gas-generating agents like sodium bicarbonate $\left(\mathrm{NaHCO}_{3}\right)$ or ammonium bicarbonate $\left(\mathrm{NH}_{4} \mathrm{HCO}_{3}\right)^{154}, 155$ Using a water-in-oil-inwater $(\mathrm{W} / \mathrm{O} / \mathrm{W})$ double emulsion method, the PLGA shelled nanoparticles were created with an aqueous core compartment containing drugs and $\mathrm{NaHCO}_{3}$ or $\mathrm{NH}_{4} \mathrm{HCO}_{3}$, which serves as the gasgenerating agent (Fig. 15). When these nanostructures are present in acidic conditions, the $\mathrm{H}^{+}$ions infiltrate into the nanostructure and induce the formation of gas $\mathrm{NBs}$ due to the reaction with $\mathrm{NaHCO}_{3}$ or $\mathrm{NH}_{4} \mathrm{HCO}_{3}$ to form $\mathrm{CO}_{2}$, which finally causes the rupture of the shells and triggers the release of the encapsulated drug.

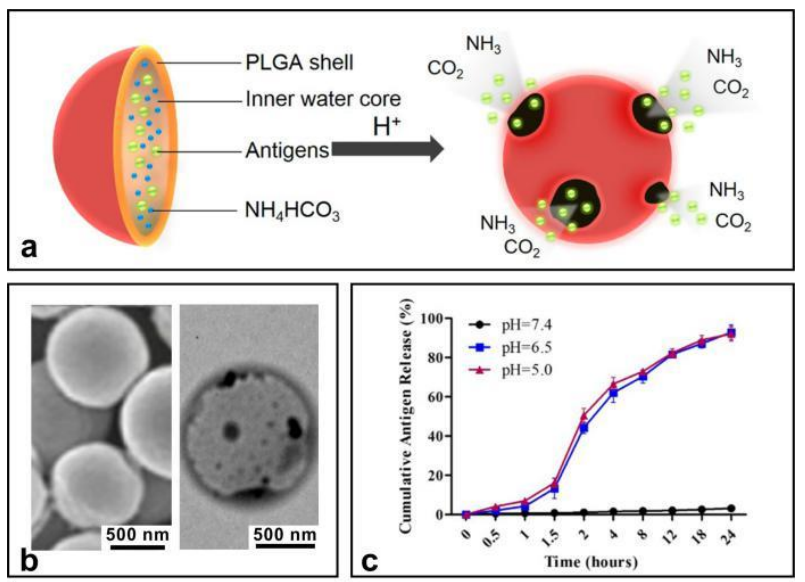

Fig. 15. (a) Schematic illustration of the structure of $\mathrm{pH}$-responsive PLGA nanoparticles and indirect NBs generated in the acid solution. (b) SEM and TEM images of $\mathrm{pH}$ responsive PLGA nanoparticles. (c) pH-responsive PLGA nanoparticles incubated in test media with different $\mathrm{pH}$ values at $37^{\circ} \mathrm{C}$. The figures are adapted from reference ${ }^{155}$.

\subsubsection{Synthesis, characterizations and biomedical applications} of $\mathrm{pH}$-responsive NBs

As the response of a NB to a change in $\mathrm{pH}$ is an intrinsic feature, $\mathrm{pH}-$ responsive NBs could be made by both physical and chemical methods. However, chemical methods are used the most to apply a suitable shell to the NBs for obtaining a specific $\mathrm{pH}$-response and to provide stability for biomedical applications (see details in section $\S 4.2$ Chemical formulations). Electron microscopy is typically used to this visualize such shelled $\mathrm{pH}$-responsive NBs, while DLS is used to measure their size (see sections $§ 4.3 .2$ Techniques with high spatial resolution, § 4.3.3 Techniques with low spatial resolution ). Both directly and indirectly $\mathrm{pH}$-responsive NBs have been used for biomedical applications. For example, direct $\mathrm{pH}$-responsive NBs have been applied for delivery of therapeutic gases, such as oxygen delivery for cancer therapy (section \$5.2.4 Delivery of therapeutic gases). Indirect $\mathrm{pH}$-responsive NBs have also been described for applications involving the controlled-release of drugs (see section $\S 5.2 .1$ Anticancer drug targeted delivery).

\subsection{Multimodal stimuli-responsive NBs}

Generally speaking, NBs are intrinsically endowed with bimodal responsiveness: they scatter light and have acoustic properties. As an example, transient NBs generated by a laser pulse irradiating hemozoin nanocrystals produce both optic and acoustic responses (Fig. 16): a bright flash in a time-resolved optical scattering image and an acoustic response, both of which can be detected in a timeresolved manner. ${ }^{156}$
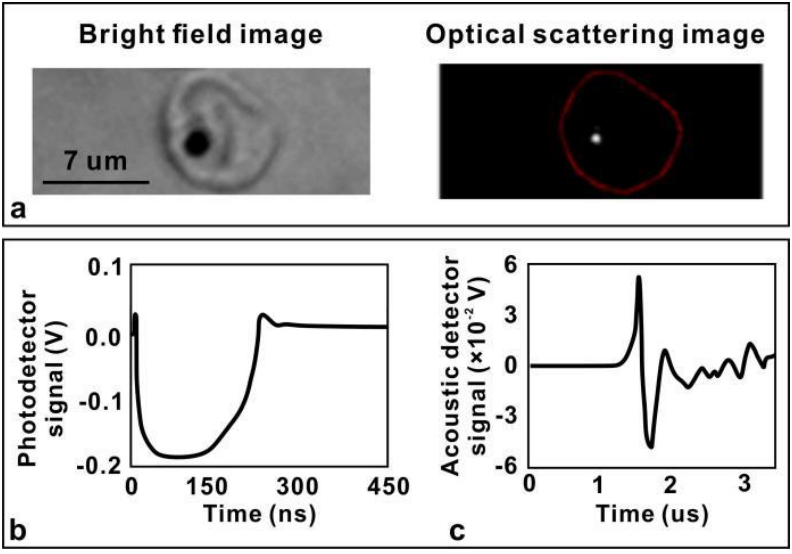

Fig. 16. (a) (left) Bright field image showing a cell before laser irradiation and (right) a darkfield image showing localized scattering from a laser-induced NB; the cell boundary is indicated in red. (b) Time-resolved optical signal of the laser-induced NB and (c) the resulting acoustic traces. The figures are adapted from reference ${ }^{156}$

In addition, NBs can be further modified to achieve additional multimodal responses. For example, Huang et al. embedded superparamagnetic iron oxide nanoparticles (SPIONs) in the shell of NBs to create a magnetic and ultrasound dual-responsive NB for multimodal imaging by ultrasound and MRI. ${ }^{140}$ Recently, similar multifunctional NBs have also been reported by others. ${ }^{157}$ Furthermore, some studies have even developed NBs with quadruple-mode responsiveness. ${ }^{142,158,159}$ Both Luo et al. and Li et al. reported the development of ultrasound-, magnetic-, and fluorescence-responsive NBs for multimodal imaging. The NBs were coated with PLGA to which a fluorescence dye and the magneticresponsive materials, such as SPIONs, were directly conjugated; or alternatively these compounds can be encapsulated in the NBs.

\section{Synthesis and characterization of stimuli-}

\section{responsive NBs}


In this section, we will first overview the methods used for synthesis of stimuli-responsive NBs and then describe the techniques applied for their characterization. Considering that the methods for formation of indirect responsive NBs have already been discussed in the previous section, we will only focus on methods used for the synthesis of direct-responsive NBs in bulk solutions. We have divided these methods into physical production and chemical formulations.

\subsection{Physical methods}

\subsubsection{Ultrasonication}

Bulk MBs/NBs can be generated by ultrasound by directly irradiating pure water solutions or aqueous solutions of surfactants. As shown in Fig. 17, the typical protocol for the generation of NBs by ultrasonication is as follows: $145,150,160,161$ (1) aqueous samples are first filtered to remove any dust or particles in the solution; (2) the solution is irradiated by ultrasonication in a frequency range of 10$1000 \mathrm{kHz}$ and an output power ranging from a few tens to hundreds of Watts; and (3) after sonication, the NBs are further selected from the bubble mixture either by centrifugation or are simply left to stand for a few hours to remove MBs, which rise more rapidly to the solution surface.

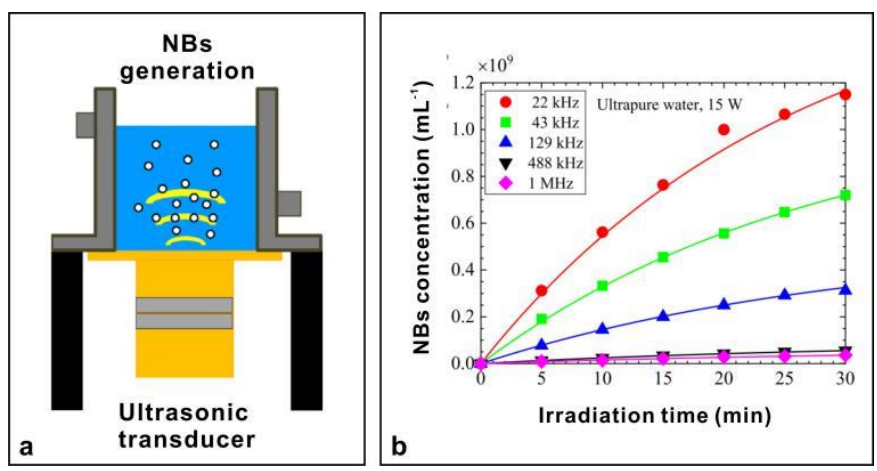

Fig. 17. (a) Schematic illustration of NBs generated by ultrasonication; (b) The effect of ultrasonic frequency on NB generation in ultrapure water at $15 \mathrm{~W}$. The figures are adapted from reference ${ }^{161}$

NBs with diameters of tens to hundreds of nanometers can be generated using this method. The ultrasonic power and frequency exert a substantial effect on the NBs concentration and diameter. Yasuda et al. found that the NBs concentration increased with increasing ultrasonic power and decreasing frequency, while the concentration of NBs also increased with irradiation time and gradually approached an equilibrium value of $\sim 1.5 \times 10^{9} / \mathrm{mL}$. ${ }^{161}$

Furthermore, bulk NBs can be generated by applying ultrasonic irradiation to previously formed MBs in liquid by inducing the collapse of MB, which results in the formation of smaller NB. ${ }^{162,163}$ Regardless of which of the above-mentioned methods is used, NBs always need to be separated from a mixture of MBs and NBs; but even then, the selected NBs still retain a multimodal size distribution.

\subsubsection{Pressure-based methods}

Based on the principles of Henry's Law, whereby the amount of dissolved gas in a liquid is proportional to its partial pressure above the liquid, pressure-derived methods can be developed to generate NBs having different types of gas cores in a liquid.

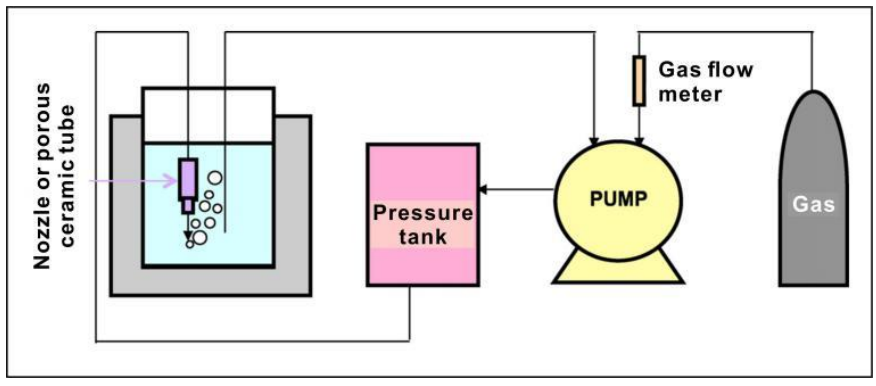

Fig. 18. Schematic illustration of NBs generated using the pressure-based method. The figure is adapted from reference. ${ }^{164}$

NB production occurs as follows (Fig. 18): (1) a gas such as oxygen, nitrogen or air is introduced and mixed with water by a pump; (2) the water and gas mixture is subjected to high pressure (typically in $0.2-$ $0.4 \mathrm{MPa}$ ) in a pressurized tank in order to increase the dissolution of gas in the water; ( 3 ) the supersaturated gas solution is depressurized under atmospheric pressure leading to the nucleation of the bubbles; (4) NBs are generated which are then expelled through a nozzle or a porous ceramic tube at the outlet; and (5) the above procedures are repeated until the desired concentration of NBs in the water is achieved. This method can produce a high concentration of NBs in large scale and has been introduced as a method of industrial generation of NBs by Iwaki Co. Ltd., Japan and by AS ONE Corporation, Osaka, Japan.

Alternatively, the pressurized gas-liquid mixing step can be omitted by simply starting from an unsaturated gas-aqueous solution at normal temperature and pressure. Upon lowering the pressure below atmospheric conditions, the solution eventually becomes a supersaturated gas solution, and extra gas molecules might be released and aggregate into bubbles. ${ }^{165}$ With this method it was found that the concentration of the NBs first increased and then decreased as a function of the decompression time, while the size of the NBs increased during this decompression process. 
Recently, there have been a few reports describing repeated

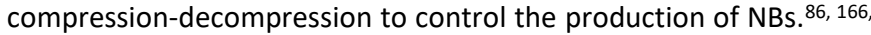
167 Bulk NBs are produced by repeatedly pressurizing gas into the solution followed by depressurization. Jin et al. suggested that MBs of about $10-50 \mu \mathrm{m}$ in size formed during the first decompression step and then decreased to become nanoscale-sized during compression. ${ }^{166}$ Wang et al. further pointed out that the size of the formed NBs decreased over time if a constant frequency of compression-decompression cycles are applied, so that sizeadjustable NBs can be formed by controlling the operating time. ${ }^{86}$

\subsubsection{Hydrodynamic cavitation}

A method of hydrodynamic cavitation has also been proposed to produce bulk NBs in high concentrations and of uniform size. ${ }^{126,168}$ 172 Hydrodynamic cavitation can occur inside a chamber tube (as shown in Fig. 19) when a gas-water solution passes through a constricted region into a region with lower pressure, leading to the nucleation of bulk NBs. According to Bernoulli's principle, an increase in the velocity of a liquid flow due to a reduction in the area leads to a decrease in liquid static pressure. When the local pressure falls below the vapor pressure of the liquid, tiny bubbles nucleate in the liquid.

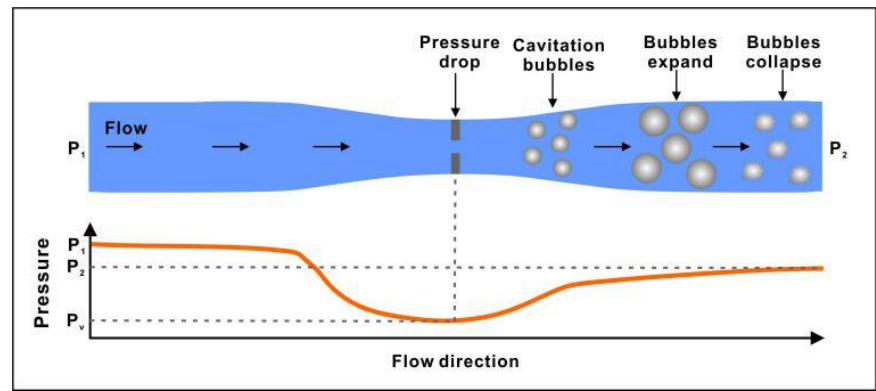

Fig. 19. Schematic of the flow and pressure distribution in a hydrodynamic cavitation device.

Based on a hydrodynamic cavitation method, Maeda et al. developed an ultrafine bubble generating system in which bubbles are generated from a gas-liquid mixture that passes through a pipe with changing cross-sectional area. ${ }^{168}$ Michailidi et al. in turn generated air and oxygen bulk NBs with a narrow-size distribution using a counterflow hydrodynamics approach. ${ }^{172}$ Pourkarimi et al. studied the effects of various factors such as gas flow, changes in pressure in the cavitation nozzle, gas types, and changes in the Venturi tube internal diameter on the concentration of NBs and the resulting diameters. ${ }^{169,} 170$ The authors found that the inlet pressure to the
Venturi tube has a significant effect on the mean bubble diameter within a certain pressure range. However, Nirmalkar et al. found that the bubble size distribution is more or less unaffected by the inlet pressure and the mean bubble diameter remains constant at $\sim 130$ $\mathrm{nm}$, but the bubble number density increases considerably with changes in operating pressure, or by using hydrodynamic cavitation with a high-pressure microfluidic device. ${ }^{126}$ The difference is likely caused by the different configurations of the Venturi tube.

\subsubsection{Mixing or shearing methods}

Bulk NBs can also be generated by mixing or shearing of the flow of a gas-liquid mixture. ${ }^{173-175}$ Based on Bernoulli's principle, and similar to the NBs generated by hydrodynamic cavitation, mixing or shearing of a fluid causes an increase in the speed of the fluid and a concomitant decrease in pressure, inducing nucleation and generation of NBs. Wu et al. reported the generation of $\mathrm{CO}_{2} \mathrm{NBs}$ by high intensity agitation and found that the size of NBs was largely dependent on the agitation speed of the impeller, but was not very dependent on the duration of the agitation step. ${ }^{176}$ NBs are generated using a blade positioned within a chamber, which cuts macro-bubbles entrained in the liquid to MBs/NBs. ${ }^{177,} 178$ In order to avoid the use of impellers or blades that may be a source of contamination, an agitation-free mixing method was developed by directly mixing $\mathrm{CO}_{2}$ gas with distilled water through an up-and-down movement using a specially designed actuator. ${ }^{174}$ Furthermore, the spontaneous generation of NBs was reported to occur by mixing warm water with cold gas-aqueous solution forming a turbulent jet flow. ${ }^{175}$ The micro/sub-micro-vortices are caused by high energy dissipation rates by discharging warm water into the cold gas aqueous solution, inducing shear stresses in these domains, and local gas supersaturation, which in turn lead to the formation of high concentrations of bulk NBs $\left(\sim 10^{9} / \mathrm{mL}\right)$.

\subsubsection{Porous membrane or medium based methods}

Another method of forming NBs is by pumping gas into a liquid which flows through a porous membrane or a porous material. ${ }^{179-182}$ Kukizaki et al. reported that monodispersed NBs with a mean bubble diameter in the range of 360-720 nm can be stably produced using Shirasu-porous-glass membranes with a mean pore diameter ranging from $43-85 \mathrm{~nm} .{ }^{179}$ It has been suggested that NB formation using a pore mainly consists of bubble expansion and bubble detachment (Fig. 20). The air-water surface tension of NBs acts as a force 
retaining the NB at the pore opening. However, the drag force caused by the water flow acts as a force to detach the NB from the pore. When the NB grows, the detaching force becomes larger than the holding force, resulting in NB detachment from the pore. Therefore, monodispersed NBs with a diameter larger than the pore diameter are released from the membrane.

Instead of Shirasu-porous-glass membranes, Kerfoot et al. developed a NB generator device based on pressurized flow through porous beads. ${ }^{181}$ Ryu et al. used a bamboo fibrous layer as a membrane made of a natural material and passed pressurized gas through the bamboo membrane at a pressure above that of atmospheric pressure, allowing the gas to permeate from inside the bamboo fibrous layer to the outside, thus generating the NBs. ${ }^{180}$

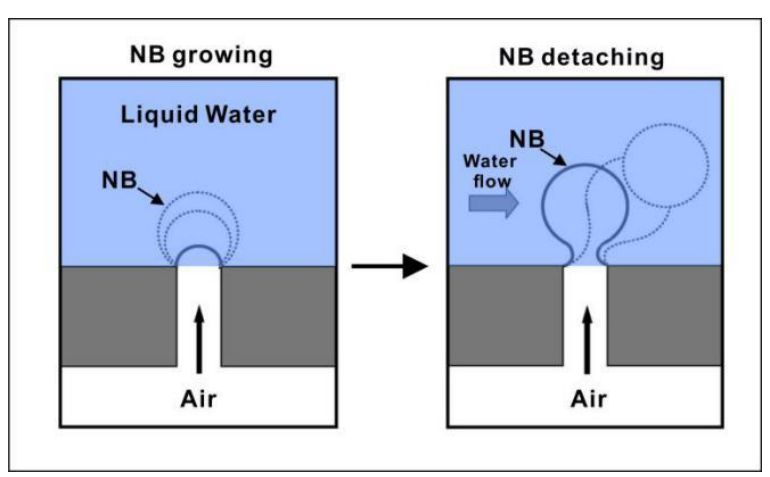

Fig. 20. Schematic illustration of NB formation process from a pore in a water flow. The figure is adapted from reference ${ }^{179}$.

\subsubsection{Solvent exchange}

Due to its simple procedure, ethanol-water solvent exchange is the most commonly used protocol to produce surface NBs. However, the solvent exchange method was recently extended to generate bulk NBs as well. ${ }^{183,} 184$ The typical steps of the solvent-exchange process are as follows (Fig. 21): (1) injection of a solvent with high gas solubility like ethanol into a fluid container, (2) injection of water (with low gas solubility) into the solvent, which results in (3) the formation of NBs in the fluid during the injection of water. The mechanism involved in the generation of NBs by solvent exchange is likely due to the fluid being locally saturated or even supersaturated with gas that is generated by the solvent exchange.

Qiu et al. reported that the concentration of bulk NBs produced by this method is about five times higher than when using degassed water alone, confirming the formation of extra bulk NBs during the ethanol-water exchange process. ${ }^{183}$ Different ratios of ethanol to water in solution can result in different gas solubilities and could further induce the generation of different concentrations of NBs. Indeed, when the ratio of ethanol to water increases from 1:1 to $1: 12$, the concentration of NBs gradually increases. However, further increasing the ratio to $1: 20$ does not increase the formation of NBs but it is decreased probably due to nitrogen saturation which first increases and then decreases upon changes in the molar fraction of water.

Similar to the concept of solvent exchange for the generation of NBs, bulk NB can also be produced by the exchange of cold water with warm water due to the temperature-dependent solubility of gas. ${ }^{2}$ For instance, air solubility in water decreases from $\sim 31 \mathrm{mg} / \mathrm{L}$ at $23^{\circ} \mathrm{C}$ to $\sim 24 \mathrm{mg} / \mathrm{L}$ at $8^{\circ} \mathrm{C}$. The advantage of this method is that does not require organic solvents, which thus avoids any potential contamination.

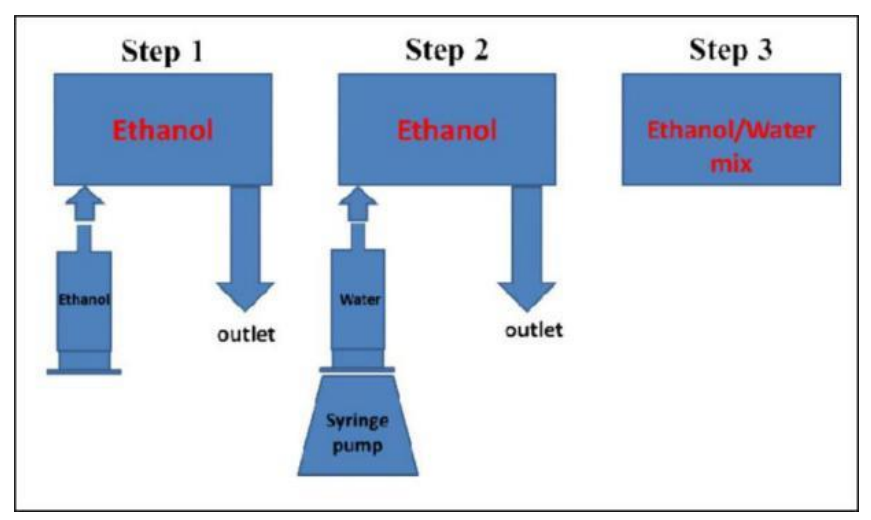

Fig. 21. Schematic illustration of the NBs generated by the ethanol-water exchange process. Step 1: injection of ethanol into a fluid container; Step 2: injection of water into ethanol; Step 3: production of NBs in the bulk fluid upon injection of water. The figure is adapted from reference ${ }^{183}$.

\subsection{Chemical formulations}

Typically, bulk generation of NBs achieved by the physical methods described above are produced without specifically coated shells, although surfactant molecules may be added to the solution in some cases and forms shells that stabilize the formation of the NBs. In this section, we will focus on methods using chemical formulations to generate NBs that are typically coated with shells to achieve stabilization and allow further modifications. The key features of the NBs formulation are the core gas and the different shell materials used as they can affect both structural and functional behaviors. Typically, gaseous cores having minimal solubility in water, such as PFCs, are used to reduce the gas dissolution from the core into the surrounding liquid environment, which can increase the life of NBs, for instance after injection in the blood stream. Conversely, the shell 
acts as a barrier between the core gas and external aqueous medium, which further prevents gas dissipation and improves the NBs stability. Various materials have been used for the shell, such as phospholipids, polymers (i.e. PLGA, polysaccharides), or proteins (i.e. albumin). The selection of shell materials is generally based on the desired physicochemical properties like charge and lipophilicity, but as well on the desired shell functionalization with specific target ligands, for instance to achieve targeted drug delivery.

\subsubsection{Hydration of a thin lipid film}

Hydration of a thin lipid film is one of the most widely used methods to fabricate shell-coated NBs. ${ }^{185}$ The thin-film hydration method typically involves the following steps (Fig. 22): (1) a phospholipid mixture (normally in different ratios of DPPA, DPPC, DPPE, DSPC, DSPE, or DSPE-PEG; respectively, 1,2-dipalmitoyl-sn-glycero-3phosphate; 1,2-dipalmitoyl-sn-glycero-3-phosphocholine; 1,2Bis(diphenylphosphino)ethane;

1,2-distearoyl-sn-glycero-3phosphocholine;

1,2-distearoyl-sn-glycero-3phosphorylethanolamine; and 1,2-distearoyl-sn-glycero-3phosphoethanolamineN-[biotinyl(polyethylene glycol)]) are dissolved in an organic solvent, typically chloroform; (2) the solvent is then removed by evaporation to obtain a dried thin-film mixture of lipids that are then hydrated in aqueous solution (such as glycerol, PBS, or saline solution) to prepare a liposomal suspension; and (3) the liposomal dispersion is finally filled with suitable gas to form bubble cores by mechanical agitation in a shaking machine or by sonication.

To improve the fabrication yield of NBs with good stability, the addition of polymers is required to form lipid/polymer hybrid shells. For instance, to reduce bubble size, Krupka et al. reported a formulation incorporating the surfactant Pluronic into the NBs lipid shell that leads to a significantly reduced bubble size. ${ }^{186}$ Liu et al reported a method of incorporating silicon hybrid lipids into the normal lipid shell of NBs to control the NBs size and found the size of the NBs could be adjusted from $\sim 500 \mathrm{~nm}$ to $\sim 850 \mathrm{~nm}$ by modulating the ratio of silicon hybrid lipids in the NBs shell. ${ }^{187}$ Hernandez et al. described a method of forming a shell with a double-layer (i.e., the inner layer formed by a crosslinked polymer mesh and the outer layer formed by pluronic/phospholipid) to increase the stability of NBs. ${ }^{188}$

In this typical protocol of thin-film hydration, post-processing is always required to separate NBs from a mixture of MBs using centrifugation, gravitational forces, physical filtration, or floatation. 189,190 For instance, in the centrifugation processing, lowspeed centrifugation is used to separate large bubbles from the suspension followed by high-speed centrifugation after the suspension is transferred to remove phospholipid fragments or liposomes. ${ }^{191,192}$ Conversely, Cai et al. optimized the thickness of the phospholipid film to directly produce uniformly sized NBs with no further need for post-processing purification or addition of amphiphilic surfactants. ${ }^{190}$

Microfluidics is well-known to be suitable for the fabrication of lipid coated MBs of uniform size and has been explored to fabricate lipid coated NBs. Peyman et al. described a microfluidic method for the generation of NBs with coated lipid shells and a perfluorobutane (PFB) gaseous core. ${ }^{193}$ NBs with an average diameter of approximately $100-200 \mathrm{~nm}$ are produced in an atomization-like flow regime at high production rates ( $>10^{6}$ bubbles per second) and at high concentration ( $>10^{11}$ bubbles per $\mathrm{mL}$ ).

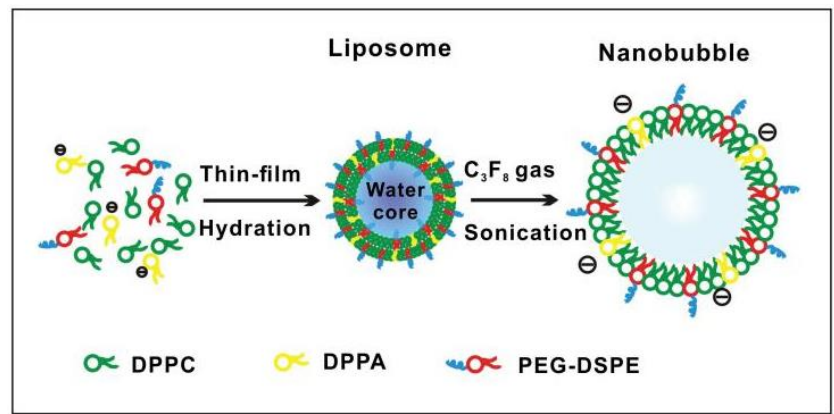

Fig. 22. Schematic presentation of the procedure of formation of NB by the thin lipid film hydration method. The figure is adapted from reference ${ }^{183}$.

In order to achieve multimodality, NBs can be loaded or functionalized with other materials post-formulation. ${ }^{194}$ For instance, to load drugs onto the NBs for therapeutic applications, a hydrophobic drug can be incorporated directly into the lipid-shell, or charged components, such as doxorubicin or nucleic acids, can be easily coupled electrostatically with anionic/cationic lipids of the bubble shell. ${ }^{195}$ In addition, molecules can also be chemically attached onto the NBs. ${ }^{157}$ As an example, Wang et al. synthesized targeted paclitaxel (PTX) containing NBs by attaching PTX to the lipid shell and conjugating an anti-ProGRP monoclonal antibody to the lipid surface. ${ }^{196}$ In another approach, the drug-containing liposomes were conjugated to lipid shell coated NBs for ultrasound-mediated drug targeted delivery of liposomes to cancer cells. ${ }^{197}$

\subsubsection{Polymer emulsification}


Polymer emulsification is also widely used to fabricate polymer shel coated NBs. A modified double-emulsion (water/oil/water) evaporation process has been suggested for organic solvent dissolving polymers like PLGA-coated NBs. ${ }^{141,} 142,198-200$ The typical process is as follows (Fig. 23): (1) aqueous solution is added to the polymer dissolved in organic solution and the mixture is emulsified typically by ultrasonic treatment to form the first emulsion; (2) the emulsified solution is added to an aqueous solution with a polymer such as poly(vinyl alcohol) (PVA) and emulsified by a homogenizer to produce a second emulsion; and (3) after extracting the organic solvent and purification, the solution undergoes a freeze-drying step to remove any aqueous solvent; the remaining emulsion matrix is filled with the suitable bubble core gas and the formed NBs are ready for use.

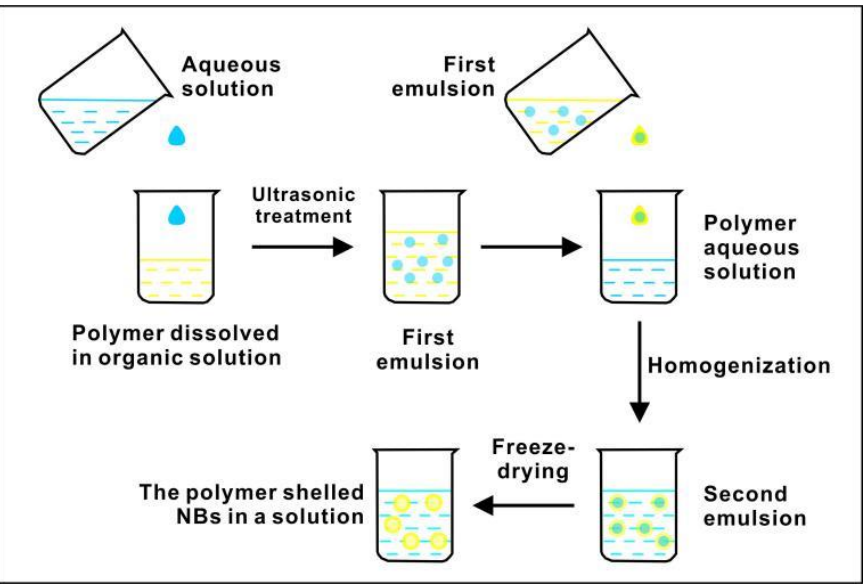

Fig. 23. Schematic presentation of shelled NBs prepared by the polymer emulsification method.

In addition, a method without the freeze-drying step was developed to obtain NBs coated with aqueous dissolvable polymers. ${ }^{201-207}$ Briefly, a pre-emulsion is prepared by mixing an ethanol solution of surfactant and co-surfactant, water, and generally PFC (liquid phase at room temperature) using a high shear homogenizer. Next, an aqueous solution of a polymer, generally a polysaccharide, is added in drops during stirring to form polymer-shelled nanodroplets. Finally, to generate the NBs, the nanodroplets are irradiated by ultrasound to trigger a phase-shift by increasing the temperature.

Recently, Song et al. reported a method combining hydration with an emulsification process to formulate acetalated-dextran polymer shelled oxygen NBs. ${ }^{153}$ Initially, a thin lipid film and lipid solution are formed using the method described above. Next, the emulsions are produced by mixing the organic solution prepared using an organic solvent in which the acetalated dextran is dissolved in the lipid solution using ultrasound. Following purification, the solution is frozen in liquid nitrogen and is further freeze-dried to remove water The lyophilized matrix is thus set in an environment of oxygen gas to obtain the oxygen NBs.

\subsubsection{Dissolution of the nanoparticle core}

Another technique used for the preparation of shell-coated NBs involves growing the shell materials on the surface of a NP which acts as nucleus that is subsequently removed by dissolution and is replaced with a gas core. As an example, Huang et al. reported a method used to grow a silica shell onto polystyrene (PS) NPs. ${ }^{208}$ The PS core is dissolved overnight in the presence of tetrahydrofuran (THF) and the NBs are generated by drying for $24 \mathrm{~h}$ under vacuum and filling the cores with air.

\subsection{Methods for characterizing size, concentration, morphology, composition, surface charge, or lifetime of NBs}

Due to their distinct properties, different techniques have been used to characterize bulk and surface NBs. For instance, AFM is the most used technique for the measurement of surface NBs, but it is not suitable for the characterization of bulk NBs as they are suspended in the bulk medium. Another example is DLS, which is rather suited to characterize bulk NBs. Herein, we will overview the main methods used for characterization of direct- or indirect-responsive bulk NBs (hereinafter simply referred to as direct or indirect NBs), considering the methods for characterization of surface NBs having been overviewed by others in recent reviews. ${ }^{19,} 22$

A growing number of techniques are available for investigating direct and indirect NBs, some of which provide a high spatial resolution like transmission electronic microscopy (TEM) or scanning electronic microscopy (SEM). With a resolution down to $\sim 0.1 \mathrm{~nm}$, electron microscopy (EM) indeed has the spatial resolution to resolve NBs of tens to hundreds of nanometers in great detail. Other methods lack such high spatial resolution but are rather sensitive to the chemical properties of the NB, such as attenuated total reflection infrared spectroscopy. A particular challenge is how to achieve high spatial resolution and at the same time good temporal resolution. It is often required to combine two or more techniques to obtain a complete picture. Here, we will not delve into the technical details, but we will focus on the strengths and limitations of the techniques.

\subsubsection{Light microscopy techniques}


Several light microscope techniques have been used to study direct or indirect NBs, including light transmission, light scattering, or fluorescence microscopy. These techniques are nonintrusive, fast, and do not require extensive preparation of samples.

One of the easiest and most straightforward light microscope techniques is transmitted light microscopy. ${ }^{137,} 175,187,189,209,210$ The NBs appear dark against a bright background because of absorption and/or refraction of the transmitted light (Fig. 24). Although light transmission in bright-field imaging may provide a simple and easy method of visualization of bulk NBs, it is not suited to resolve submicron bubbles clearly due to the resolution limit of optical microscopes.
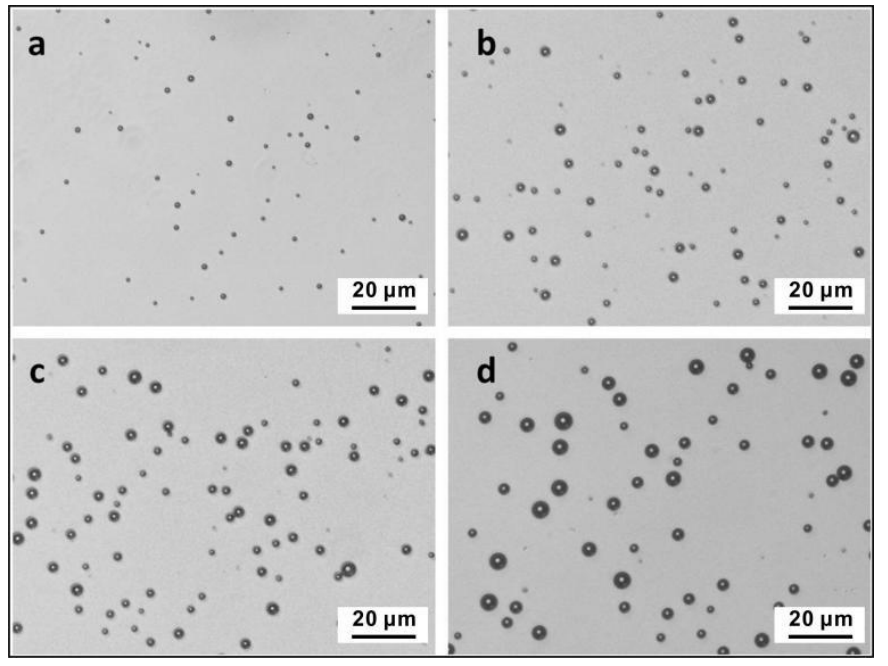

Fig. 24. Bright-field images of NBs with a size of (a) $523 \pm 46 \mathrm{~nm}$, (b) $610 \pm 53 \mathrm{~nm}$, (c) 857 $\pm 83 \mathrm{~nm}$, and (d) control MBs of $1317 \pm 37 \mathrm{~nm}$, respectively. The images are adapted from reference ${ }^{187}$.

A light scattering technique like dark-field microscopy is widely used to visualize both direct and indirect NBs. Here the NBs appear bright against a dark background (Fig. 25a). While still limited by optical resolution, the excellent contrast of dark-field imaging enables imaging the Brownian motion of individual bulk NBs for quantification of their size distribution even below the optical resolution. In the scientific literature, this is referred to as single particle tracking, but is dubbed NTA in its most frequently used commercial variant (Fig. 25b). Recently, a holographic nanoparticle tracking analysis (HNTA) has been proposed that takes into account the phase shift of the scattered light. HNTA is reported to be a powerful new method to detect NBs both in terms of size and refractive index to differentiate them from solid particles or oil droplets in the same solution. ${ }^{211}$ This technique is not only used to directly visualize bulk NBs, but it is also used to detect indirect NBs.
Many studies have reported visualization of short-lived indirect NBs (lifetime <1 $\mu \mathrm{s}$ ) generated from photothermal NPs irradiated by pulsed laser light, which can be detected by dark-field microscopy through carefully timed image acquisition with respect to the laser pulse. While this allows acquiring a single image that confirms the formation of NBs, it does not allow capturing of NB dynamics because they typically have already disappeared by the time that a second image can be acquired. For time-resolved measurements of NBs, e.g. to measure their lifetime, instead a fast point detector is used that captures the scattered light of a single NB as a function of time. ${ }^{212}$

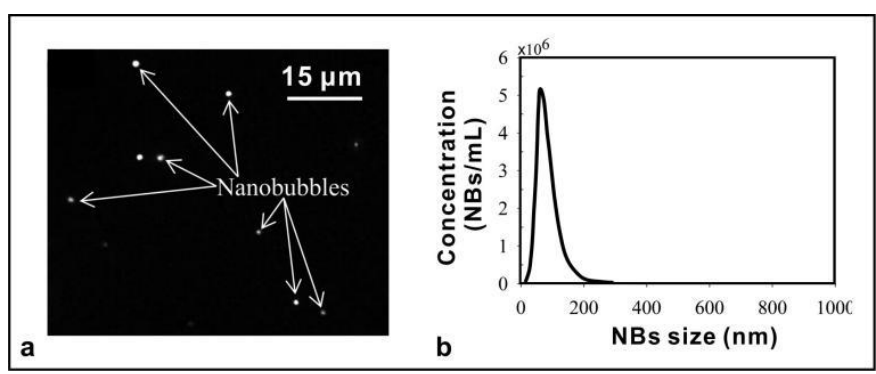

Fig. 25. NBs size measured by nanoparticle tracking analysis based on analyzing NB Brownian motion in light scattering movies. (a) An exemplary image of NBs which are visible by light scattering; (b) Size distribution of NBs, showing a single narrow peak and average and a mean size of $89 \pm 10 \mathrm{~nm}$. The figures are adapted from reference ${ }^{174}$.

Fluorescence microscopy is typically used to confirm the presence of a shell surrounding shell-coated NBs. For instance, Yin et al. reported the use of the fluorescent probe Dil to label the phospholipid shell of NBs and used confocal laser scanning microscopy (CLSM) to determine the location of red fluorescently dyed NBs following uptake by cells (Fig. 26). ${ }^{191}$ Bessone et al. reported that curcuminoids-loaded in NBs could be detected by fluorescence microscopy using curcuminoids as a bi-functional fluorescent probe and therapeutic drug. ${ }^{207}$

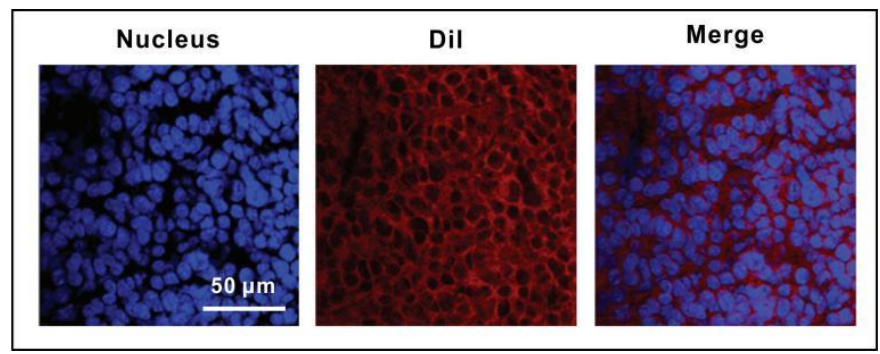

Fig. 26. Confocal laser-scanning microscopy images of tumor cells with uptake of lipid shelled NBs labeled with Dil. The images are adapted from reference ${ }^{191}$.

\subsubsection{Techniques with high spatial resolution}

Both TEM and SEM have been widely applied to visualize the structure and morphology of NBs with high spatial resolution. 
However, this is limited to very stable NBs that can withstand EM sample processing. For instance, using SEM, Huang et al. clearly visualized thin silica shelled NBs with small NP super-paramagnetic iron oxide (SPIO) attached on the shell surface (Fig. 27). ${ }^{208}$ Li et al. observed the morphology of NBs by TEM and found that NBs clearly presented a spherical shape with a distinct hollow structure and a shell thickness of approximately $10 \mathrm{~nm}$ (Fig. 27). ${ }^{142}$

Cryo- or freeze-fracture EM offers the advantage of being able to visualize a specimen in a nearly native state. This feature allows to examine the structure and morphology of intact NBs with high spatial resolution. Hernandez et al. demonstrated that Cryo-EM could be used to image nanoscale lipids and polymer-stabilized PFC gas NBs before and after their destruction with high intensity ultrasound. ${ }^{188}$ Furthermore, using a cryo-TEM, lijima et al. examined the morphology of uncoated oxygen NBs suspended in water. ${ }^{213}$ Using freeze-fracture TEM, Wang et al. reported the existence of nitrogen, oxygen, and $\mathrm{CO}_{2} \mathrm{NBs}$ without shell coating produced using a periodic pressure change. ${ }^{86}$
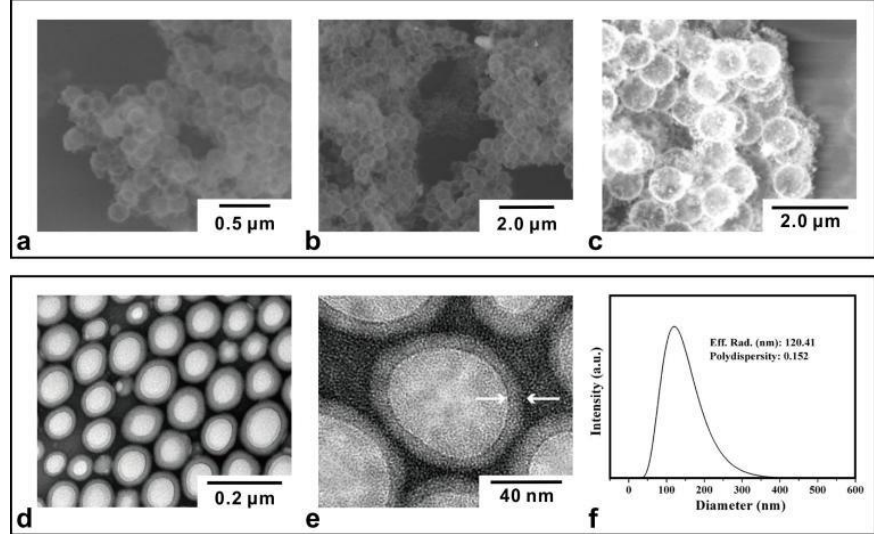

Fig. 27. (a-c) SEM images of silica-shelled NBs with diameters of (a) 200, (b) 500, and (c) $1000 \mathrm{~nm}$. (d-f) TEM images of PLGA-shelled NBs at different magnifications (d) and (e) and (f) hydrodynamic size of NBs measured by DLS. The figures are adapted from references. ${ }^{214}$

Due to its limited speed, EM imaging is only suitable for a limited number of samples which may not be sufficient to obtain a decent statistical analysis of the total population. Typically, electronic microscopy techniques are always combined with other techniques such as DLS (see next section) or NTA to quantify the size distribution of large numbers of NBs (Fig. 27f)

X-ray scattering has been applied to characterize the morphology of NBs with high spatial resolution as well. Hirai et al. explored smalland wide-angle X-ray scattering (SAXS, WAXS, respectively) to investigate the structure of NBs with air, oxygen, or nitrogen as the gas core. ${ }^{215}$ Based on X-ray scattering and modelling, they found that NBs present a dynamic diffusive boundary (interface) due to the continuous release and absorption of gas (Fig. 28). Furthermore, Xray scattering was also employed to characterize the dynamics of indirect NBs. Plech et al. developed time-resolved X-ray scattering to explore the formation of indirect laser-induced NBs from AuNP, and determined their formation was caused by a spinodal decomposition at the particle-liquid interface..$^{90,216}$ In addition, based on absorption X-ray spectra, not only can the NB morphology be determined, but also its gas core composition. Recently, Zhou et al. used synchrotronbased scanning transmission X-ray microscopy (STXM) to provide evidence of highly condensed oxygen gas molecules trapped in the NBs. ${ }^{217}$

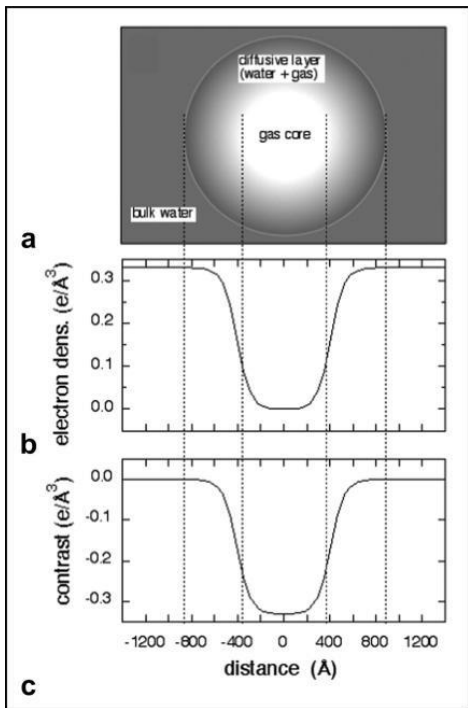

Fig. 28. (a) Schematic representation of the structure of the NB model with a diffusive boundary due to continuous release and absorption of gas at the gas-liquid interface; (b) radial distribution function of the electron density of NBs in aqueous solution; and (c) contrast (average excess electron density) profile of the NBs ${ }^{215}$

\subsubsection{Techniques with low spatial resolution}

Other techniques are available that lack spatial resolution, but instead provide physicochemical information regarding the NBs. One of the most used techniques is DLS, which analyzes Brownian motion to determine the size distribution of NBs in solution. In addition, in combination with an oscillating electric field, the zeta potential of NBs can also be calculated. However, being based on light scattering alone, DLS is sensitive to nano-sized contaminants which may skew the obtained results. Therefore, in order to confirm that the measured NPs are actual NBs, this method needs to be combined with other methods like acoustic based methods. Recently, Gnyawali et al. developed an acoustic based flow cytometry to detect single 
NBs. ${ }^{218}$ They found that the amplitude of the detected ultrasound backscatter signal is dependent on the NBs size, indicating that the acoustic flow cytometer has the potential to determine the size of individual NBs. In addition, this technique can also be applied to detect the formation of indirect NBs based on the acoustical timeresponse obtained with an ultrasound detector.

Coulter counting, an analytical electrochemical technique, is also suitable for the detection of both direct and indirect NBs. ${ }^{52,219}$ This technique works by applying a voltage between two electrodes on either side of a cylindrical pore through which the NBs can flow. As a NB enters the pore, it displaces the electrolyte buffer and therefore increases the resistance of the pore momentarily, which is witnessed by recording the current passing of the NB through the pore. The NBs size or concentration can be derived from the change in electrical current.

The resonant mass measurement (RMM) technique has also been proposed to characterize the size and concentration of NBs. ${ }^{220}$ This technique can further distinguish gas-filled NBs and similarly sized solid contaminates or droplets, based on the measurement of the buoyant mass of particles in a liquid passing through the channel. In comparison with other sizing techniques as mentioned above, including DLS or NTA, RMM may provide a more accurate size distribution or concentration of NBs in a bulk solution.

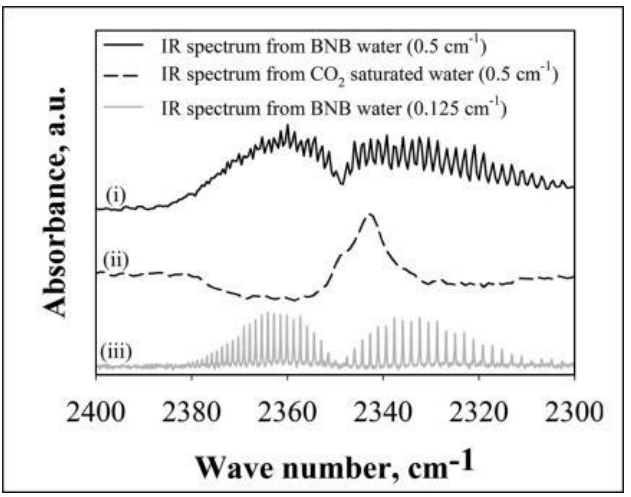

Fig. 29. ATR-FTIR spectra of (i) NBs in water, (ii) $\mathrm{CO}_{2}$-saturated water, and (iii) highresolution $(0.125 \mathrm{~cm}-1)$ spectrum of NBs. The figure is adapted from reference ${ }^{174}$.

As an additional characterization method, attenuated total reflection-frustrated total internal reflection (ATR-FTIR) can be used to identify the gas core composition. To detect NBs in solution by ATR-FTIR spectroscopy, the gas inside the NBs must be IR-active. Many gases, such as $\mathrm{CO}_{2}$ or water vapor, can strongly absorb energy from infrared radiation. $\mathrm{CO}_{2}$ is particularly suited for IR techniques because gaseous $\mathrm{CO}_{2}$ and dissolved $\mathrm{CO}_{2}$ have very different IR spectra (Fig. 29). ${ }^{174}$ Conversely, gas chromatography is also suitable for the quantification of gas in NBs. ${ }^{221}$ For a chromatography measurement, the NBs should first be disrupted in capped vials and then subjected to chromatography analysis.

\section{Biomedical applications}

Responsive NBs, like those that can be stimulated by ultrasound, have received increasing interest as they might be more promising for biomedical applications than MBs. As an example, NBs might be considered drug carriers for intravenous injections, as these small bubbles might diffuse from the blood vessels into surrounding tissues more readily; while, accumulation into tissues could possibly be further aided by antibody functionalization of the bubble surface facilitating the binding of the NB to the cell target. ${ }^{222-224}$

In this section, we will overview the application of direct- and indirect-responsive NBs in biomedicine. Specifically, we will first review bio-imaging applications including ultrasound, molecularlytargeted, and photoacoustic imaging approaches. Next, we will overview the use of direct- or indirect-responsive NBs for targeted drug delivery with special attention for the delivery of anticancer drugs and nucleic acids. Finally, we will discuss the use of direct- or indirect-responsive NBs to destroy or ablate biological tissues.

\subsection{Bio-imaging}

\subsubsection{Ultrasound imaging}

Ultrasound imaging enables cheap, non-invasive imaging in real-time with a high soft tissue contrast and without exposing the patient to harmful radiation. While in many cases additional contrast agents (CAs) like MBs or NBs are not required for poorly vascularized tumors or regions with many small vessels with limited blood flow, Doppler ultrasound by itself is not sufficient. Hence, ultrasound contrast agents (UCAs) are needed to further improve cancer detection and tumor characterization. ${ }^{225,} 226$

The majority of MBs used as UCA have diameters ranging between 1 and $10 \mu \mathrm{m}$. However, due to their large size, such MBs are unable to leave the vasculature, even in solid tumors which often have leaky vasculature. For this reason, NBs smaller than $1 \mu \mathrm{m}$ have attracted increasing attention as they are able to extravasate into the tumor tissue. In the first study on NBs used as UCA, Wheatley et al. fabricated Span 60 and Tween 80 surfactant-stabilized NBs with a gas core consisting of PFC and found that NBs were able to produce an enhanced backscatter with a maximum enhancement of 
approximately $20-25 \mathrm{~dB}$ in vitro. ${ }^{25,} 26$ Subsequently, Fan et al. compared ultrasound contrast effects of NBs and MBs in vitro and in gastric cancer xenografts. ${ }^{223}$ While the NBs showed similar mean signal intensities as MBs in vitro, due to the strong ability to penetrate the tumor tissue, the NBs provided superior contrast of the xenografted tumor for a longer time compared with the MBs (Fig. 30). Recently, similar results were reported by Zhang et al. who performed both an in vitro study and in vivo tumor imaging study using nude rats transplanted with breast cancer cells. ${ }^{227}$ The in vitro studies suggested that both the NBs and SonoVue (a commercial MB) had a similar image enhancement capability. However, the in vivo studies revealed that NBs provided an enhanced tumor intensity compared to MBs.

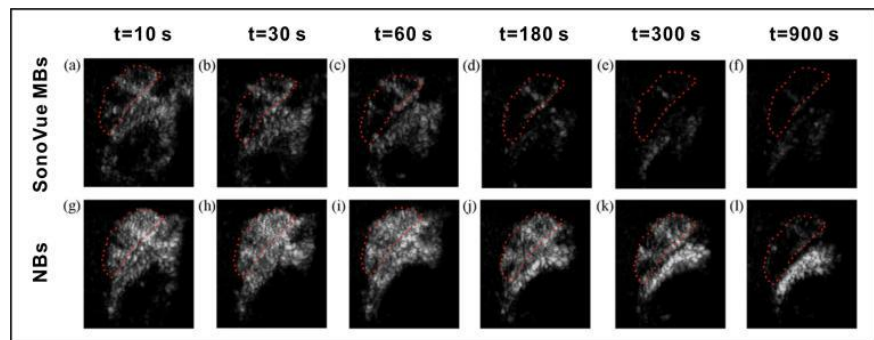

Fig. 30. Ultrasound contrast-enhanced images with the SonoVue MBs and NBs in the same gastric cancer xenograft. Panels (a)-(f) and (g)-(I) represent ultrasound contrast images of SonoVue MBs and NBs at 10, 30, 60, 180, 300, and $900 \mathrm{~s}$ after injection, respectively. The red regions indicate transplanted tumor. The images are adapted from reference ${ }^{223}$.

Similar to the strategy used to stabilize MBs, both soft-shell and hardshell NBs were developed with enhanced stability and circulation duration in vivo. ${ }^{228}$ Typically, soft-shells are not based on covalent bonds, but rather on hydrophobic interactions. Common soft shell materials include surfactant molecules or phospholipids. ${ }^{229} \mathrm{Hard}$ shells instead mainly consist of polymers (like PLGA, PVA, or polysaccharides) or denatured proteins (like albumin), as well as silica materials. Generally, the hard-shell NBs exhibit better stability and are preferred CAs for higher-intensity ultrasound applications, where they provide a higher echogenicity than soft-shell NBs which might rupture. Although most shelled NBs show extended stability in vitro, they do not perform as well when continuously irradiated with ultrasound or used in vivo because UCAs undergo fast and extreme oscillations in which gas leaks out during expansion and shell materials are shed during compression. ${ }^{229,} 230$

In order to increase circulation half-lives of NBs in vivo, several strategies have been developed. First, phase-change nanodroplets have been proposed as a solution designed to enhance the metastability of the nanodroplet; the core is in the liquid state upon injection until it is activated by ultrasound energy to vaporize and expand, resulting in nanoscale or microscale gas bubbles. ${ }^{231}, 232$ When designed properly, these nanodroplets can exhibit greater stability in the circulation. ${ }^{82,233,234}$ Second, solid cavitation agents such as nanocups, nanocones, and porous structures are capable of trapping gaseous bubbles on the surface of the hydrophobic nanostructure. ${ }^{75}$ For example, silica based porous NPs have hydrophobic inner pores with a hydrophilic outer surface. ${ }^{235}$ In order to improve biocompatibility and reduce particle aggregation, the outer surface of the porous NPs was PEGylated, ${ }^{236}$ which resulted in particles with diameters of approximately $150 \mathrm{~nm}$ and a pore size of $12 \mathrm{~nm}$. The hydrophobic pores trapping the gas for the cavitation nucleation step reduced the cavitation threshold acoustic pressure amplitudes, and thus allowed the nucleating cavitation step to be repeated hundreds of times, which is superior to polymer or lipid shelled NBs. Finally, as has been astutely suggested ultra-stable NBs can be designed by engineering the bubble membrane shell to be able to withstand deformations induced by ultrasound irradiation and flow shear forces in vivo. Leon et al. reported that a specific membrane bilayer architecture with contrasting elastic properties can redistribute stress, dissipate excess energy, and deform with no resulting irreversible damage, minimal gas leakage, or membrane shedding during insonation, and therefore may provide better persistence as it circulates within the body. ${ }^{237}$

\subsubsection{Molecular imaging}

Molecularly-targeted NBs offer the opportunity to improve the ability of ultrasound imaging to distinguish pathological tissue from healthy tissue. The surface of targeted NBs requires functionalization with aptamers or other ligands, which can selectively and actively target disease antigens and provide new opportunities to improve the diagnostic capabilities of ultrasound imaging. ${ }^{238-240}$

Such molecularly-targeted NBs have been applied to detect cancer cells overexpressing a specific antigen. For instance, two independent studies have reported prostate specific membrane antigen (PSMA, overexpressed in prostate cancer)-targeted-NBs as a novel ultrasound contrast agent for the diagnosis of prostate cancer. ${ }^{241,242}$ As another example, Yu et al. developed targeted NBs by coupling anti-G250 nanobodies to lipid-shell NBs to target renal cell carcinoma cells for ultrasound molecular imaging and diagnosis of renal tumors. ${ }^{243}$ Yang et al. conjugated NBs with Affibody 
molecules with specific affinity to HER2-overexpressing tumors. ${ }^{244}$ Affibody-conjugated NBs showed good ultrasound enhancement, and demonstrated a peak intensity of $104.5 \pm 2.1 \mathrm{~dB}$ under ultrasound contrast scanning. Zhang et al. demonstrated that PLGAshelled NBs conjugated with tumor-targeting monoclonal anti-HLA$\mathrm{G}$ antibodies enhance the ultrasound imaging of the targeted JEG-3 cells overexpressing human leukocyte antigen G (HLA-G). ${ }^{200}$

Apart from tumor targeting, molecularly-targeted NBs have also been reported for the diagnosis of inflammatory diseases. Zhang et al. conjugated lipid shell-NBs with anti-vascular endothelial growth factor (VEGFR)-2 ligands via the noncovalent biotin-avidin linker method for in vivo ultrasound imaging to detect rabbit abdominal aorta atherosclerotic plaques. ${ }^{245}$ The authors found that anti-VEGFR2 ligands- conjugated NBs could facilitate site-specific recognition of atherosclerosis and provided unique advantages in targeted ultrasound molecular imaging.

\subsubsection{Photoacoustic imaging}

Photoacoustic (PA) imaging is an emerging biomedical imaging modality, which allows imaging optical absorbers of tissue using acoustic detectors. Such a technique has immense potential for clinical translation since it allows high resolution, sufficient imaging depth, accommodates diverse endogenous and exogenous contrast agents, and is free from ionizing radiation. ${ }^{246}$ The contrast achieved is based on the absorption of light, which induces pressure or heat to generate PA signals. PA imaging produces a map of the initial acoustic pressure distribution, or absorbed energy density, in tissue that is irradiated by a short laser pulse. The technique enables to quantify the concentrations of specific contrast agents that attach to the pathological site. Light-absorbing PA contrast agents have been extensively explored to boost the detection sensitivity or the targeting specificity of molecular PA imaging. ${ }^{247}$

Pre-existing direct-responsive NBs have been explored to improve PA imaging resolution and sensitivity. Kim et al. fabricated PLGA-shelled NBs encapsulating optical absorbing agents such as India ink via a modified double emulsion process to amplify PA imaging. ${ }^{248}$ India ink NBs embedded in a gelatin phantom are clearly visible in a concentration-dependent manner using a photoacoustic macroscope even when a piece of $18-\mathrm{mm}$ thick chicken breast tissue is placed on the top of the phantom (Fig. 31). Recently, Bodera et al. reported Sudan Black B stained NBs modified with antibodies directed against Herceptin for the photoacoustic imaging of BT-474 breast cancer cells. ${ }^{249}$ The results showed PA signals were only detected from cells when targeted NBs were used, but not the control group without targeting ligands.

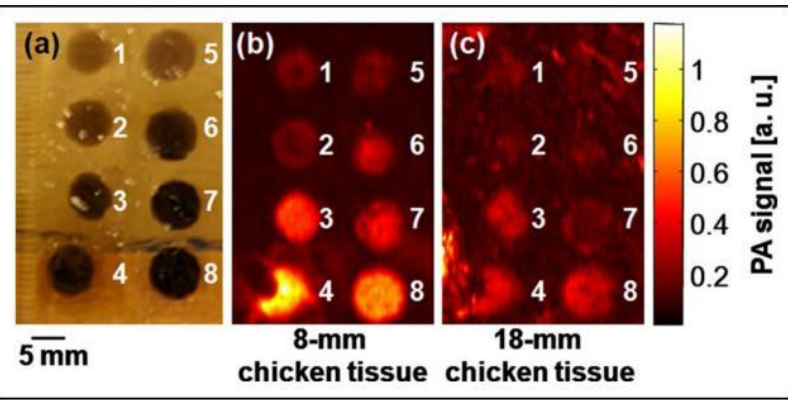

Fig. 31. Photoacoustic of image of India ink NBs. (a) Photograph of a phantom containing $\mathrm{MBs}$ and NBs at various concentrations. 1 through 4: MBs at concentrations of 2.5, 5.0 10 , and $15 \mathrm{mg} / \mathrm{mL}$, respectively. 5 through 8 : NBs at concentrations of $2.5,5.0,10$, and $15 \mathrm{mg} / \mathrm{mL}$, respectively. (b) The corresponding photoacoustic image of the phantom underneath 8-mm chicken breast tissue. (c) The corresponding photoacoustic image of the phantom covered by $18-\mathrm{mm}$ chicken breast tissue. The images are adapted from reference ${ }^{248}$.

Transient indirect-responsive NBs have also been reported to be good amplifiers of photoacoustic imaging signals since the dynamics of transient NBs are able to induce significant nonlinearly-enhanced PA signals. ${ }^{250-252}$ Transient NBs have been shown to improve detection limits and imaging contrast of quantum dots, ${ }^{253}$ circulating melanoma cells in blood, ${ }^{254}$ carbon nanotubes in the lymph, 255 bacteria with intrinsic pigment (carotenoids), and of silica-magnetic NPs in the blood, ${ }^{256}$ and carbon nanotubes in plants. ${ }^{257}$ Zharov et al. reported nonlinearly amplified PA signals obtained from transient NBs generated by overheating melanin nanoclusters in melanoma cells. ${ }^{251}$

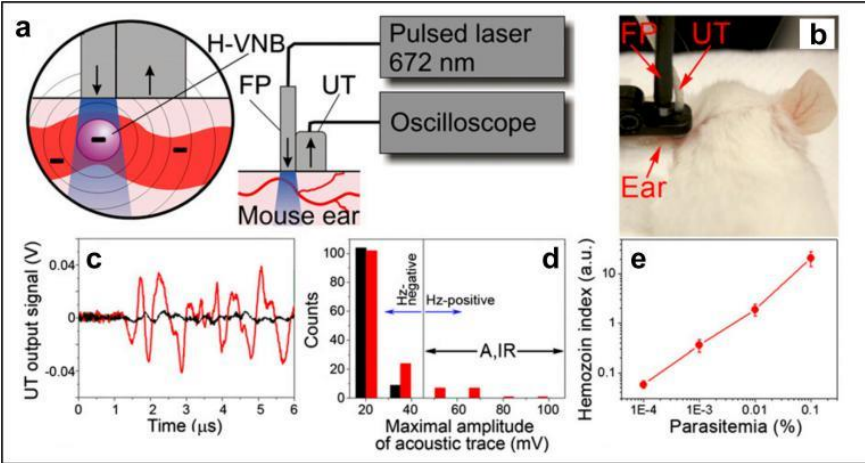

Fig. 32. (a) A probe for the generation and detection of NBs from hemozoin crystals in blood vessels (FP, fiber probe; UT, ultrasound transducer). (b) The probe placed on a mouse ear. (c) PA traces obtained for uninfected whole blood (black) and bloodhemozoin mixture (red). (d) Histograms of the maximal amplitude of PA traces obtained for uninfected whole blood (black) and whole blood-hemozoin (red) samples. (e) The hemozoin index as a function of the hemozoin-equivalent parasitemia level for hemozoin-whole blood samples. The figures are adapted from reference ${ }^{216 .}$ 
Conversely, Lapotko et al. described PA signals generated from transient NBs surrounding hemozoin crystals in response to short near-infrared picosecond laser pulses. ${ }^{156}$ The acoustic signals of these malaria-specific crystals provided a transdermal, non-invasive, and rapid detection method for malaria infection in animals without using any reagents or drawing blood (Fig. 32).

\subsubsection{Multimodal imaging}

By proper modification of the shell or core, multimodal NBs can be generated for use in a wide range of imaging techniques such as fluorescence imaging, PA imaging, or MRI. Multimodal imaging allows acquisition of additional biological information, thus increasing the precision and accuracy of disease diagnosis and prognosis. ${ }^{258-260}$

Xu et al. developed a NB contrast agent for cancer targeting and dualmode imaging using optical and ultrasound modalities. ${ }^{198}$ The authors fabricated a NB contrast agent by encapsulating the Texas Red dye in PLGA-shelled NBs and conjugated NBs with cancertargeting ligands. Simultaneous fluorescence and ultrasound images could be recorded with a linear intensity response over a large range of NB concentrations (Fig. 33). Conversely, Li et al. developed NBs with embedded SPIONs for dual mode ultrasound and MRI of neural stem cells for regenerative applications. ${ }^{261}$

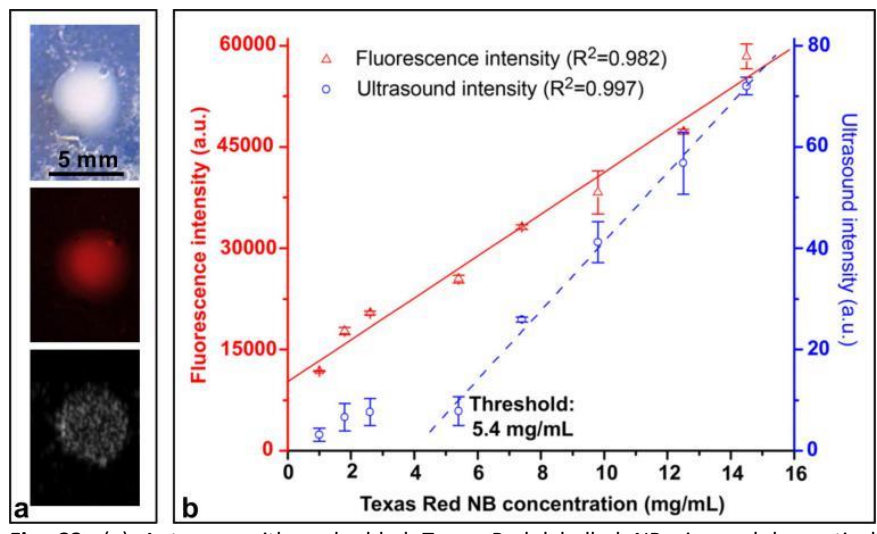

Fig. 33. (a) A tumor with embedded Texas Red labelled NBs imaged by optica photography (top panel), fluorescence (middle panel), and ultrasound (bottom panel). (b) Fluorescence intensities (red triangle) and ultrasound intensities (blue circle) measured on tumors using Texas Red NBs at different concentrations. Linear correlation is observed between the fluorescence intensity and the NB concentration ranging from $1 \mathrm{mg} / \mathrm{mL}$ to $14.5 \mathrm{mg} / \mathrm{mL}$. The ultrasound intensity is linearly correlated with a NB concentration above the threshold concentration of $5.4 \mathrm{mg} / \mathrm{mL}$. The figures are adapted from reference ${ }^{198}$

Further, a few studies have also reported triple-mode imaging with multimodal NBs. For example, Luo et al. developed NBs with the fluorescence dye DiR and SPIONs embedded in the PEG-PLGA shell for ultrasound/MRI/fluorescence imaging. ${ }^{158} \mathrm{Li}$ et al. in turn reported a multimodal PLGA-shell NB for combined NIR/MRI/ultrasound imaging of tumors by electrostatic absorption of oleylamine-/IR-780loaded hollow structures and the GdDTPA-BSA@5-FU complex to the NB shell together with folate as a targeting ligand. ${ }^{142}$ Wang et al. demonstrated the use of indocyanine green-loaded NBs for ultrasound, PA, and fluorescence multimodal imaging of prostate cancer. ${ }^{159}$ The ultrasound, PA and fluorescence signal strength correlated positively with the NB concentration in vitro. Ultrasound and PA imaging significantly enhanced the detection of tumor xenografts in vivo.

\subsection{Drug delivery}

\subsubsection{Anticancer drug targeted delivery}

Both direct- and indirect-responsive NBs have been explored for anticancer drug delivery to improve the chemotherapeutic effect. For example, Nittayacharn et al. studied doxorubicin (Dox)-loaded direct-responsive NBs in combination with low-frequency, highenergy ultrasound-stimulation as antitumor therapy. ${ }^{262}$ The authors found that drug-loaded ultrasound-responsive NBs exhibit significantly higher intracellular uptake and therapeutic efficacy compared to the free drug in vitro, and showed significantly higher accumulation and better distribution of Dox in tumors in vivo. Similar results were also obtained by other groups. ${ }^{263,264}$ In order to further improve the cellular uptake of anticancer drugs and achieve an enhanced chemotherapeutic effect, Li et al. developed Dox conjugated with cell-permeable peptides (CPP), which were entrapped in shelled NBs. ${ }^{265}$ Under ultrasound stimulation, CPP-Dox was released from NBs and penetrated into cancer cells. The authors found this system could powerfully inhibit tumor activity both in vitro and in vivo. A similar result and concept was also reported by Xie et al., but using CPP-conjugated camptothecin (CPT). ${ }^{266}$ Thakur et al. reported that ultrasound-direct-responsive NBs could also be applied to enhance intravitreal drug migration. ${ }^{267}$ The authors fabricated lipid-shelled direct-responsive NBs labelled by rhodamine, which was injected into bovine and porcine eyes ex vivo. The ultrasoundtriggered administration enhanced the directional migration of NBs in both ex vivo models, with multiple corneal ultrasound cycles promoting greater migration of NBs to the posterior regions of the vitreous body. 
Shelled ultrasound-direct-responsive NBs have also been explored for molecularly-targeted anticancer drug delivery. Zhang et al. explored PLGA-shelled direct-responsive NBs as a targeted drug carrier. They formulated methotrexate (MTX)-loaded NBs filled with a PFC gas core using a double-emulsion evaporation method. ${ }^{200}$ To target the cancer cells, the active tumor-targeting monoclonal antiHLA-G antibodies (mAbHLA-G) were further conjugated onto the surface of the NBs. The authors found that the modified directresponsive NBs could be targeted toward tumor tissues, both in vitro and in vivo, achieving enhanced targeted therapy with on-demand release of MTX triggered by ultrasound. Recently, Zhong et al. reported PTX-loaded ultrasound-direct-responsive NBs covalently conjugated with an anti-HER2 monoclonal antibody on the surface as a targeting moiety. ${ }^{268}$ These NBs significantly improved the cell targeting capability in vitro. Under ultrasound stimulation, PTX release was facilitated as was the uptake and induction of cell apoptosis in vitro. Applied in vivo, these modified NBs enhanced PTX targeting and accumulation in breast cancers, while reducing the distribution of PTX to healthy organs.

Further, light-indirect-responsive NBs have also been extensively explored. Lukianova-Hleb et al. reported an indirect-responsive NB generated from antibody conjugated AuNPs using a short near-IR laser pulse excitation to selectively permeabilize the cancer cells in and enhance intracellular delivery of a co-administered anticancer drug in vitro. ${ }^{269}$ The same group reported that anticancer drugs can be directly and selectively delivered into the cancer cell's cytosol using this light-indirect-responsive NB approach to significantly enhance the effect of chemoradiation in vivo. ${ }^{270}$

\subsubsection{Nucleic acid delivery}

Intracellular delivery of nucleic acids like plasmid DNA (pDNA), messenger RNA (mRNA), and small interfering RNA (siRNA) into the cell's cytosol is an essential step for these agents to exert their biological effects.

Direct-responsive NBs have been proposed to efficiently deliver nucleic acids to the cell's cytoplasm, especially in combination with ultrasound. Suzuki et al. have reported that gene uptake was limited to the area exposed to the ultrasound, indicating that ultrasounddirect-responsive NBs could be used to increase DNA transduction at the selected site. ${ }^{271}$ Horie et al. reported that pDNA encoding TNF- $\alpha$, encapsulated in lipid-shelled direct-responsive NBs with an octafluoropropane $\left(\mathrm{C}_{3} \mathrm{~F}_{8}\right)$ gas core, can be transfected into tumor cells by ultrasound stimulation. ${ }^{272}$ In another study, Abdalkader et al. revealed that pDNA-loaded onto direct-responsive NBs in combination with ultrasound irradiation showed significantly higher transfection efficacy in colon C26 cells in vitro. ${ }^{209}$ Furthermore, in vivo gene transfection in the left limb muscles of mice, revealed notable local transfection following ultrasound irradiation.

siRNA-loaded into direct-responsive NBs with ultrasound stimulation has been widely explored in gene silencing to enhance therapy efficiency. Yin et al. reported ultrasound- direct-responsive NBs bearing siRNA as tumor therapy by targeting the anti-apoptosis gene SIRT2. The authors found that siRNA-loaded NBs with ultrasound stimulation enhanced the gene silencing effect both in vitro and in vivo, resulting in a remarkable increase in cancer cell apoptosis. ${ }^{273}$ Subsequently, they further reported ultrasound-direct-responsive NBs composed of encapsulated chemotherapeutic agent PTX and siRNA targeting the $B C L 2$ gene ( $B C L 2$ siRNA) for the treatment of hepatocellular carcinoma. ${ }^{274}$ Wang et al. reported androgen receptor-loaded siRNA NBs for the treatment of androgenindependent prostate cancer. ${ }^{275}$ Further, Wu et al. presented antitumor targeted FoxM1 siRNA-loaded direct-responsive NBs for therapy of prostate cancer. ${ }^{276}$ Overall, siRNA-loaded directresponsive NBs combined with ultrasound stimulation significantly inhibited the expression of the targeted protein and achieved promising therapeutic results.

In addition, light-indirect-responsive NBs have also been well explored to directly deliver nucleic acids to the cell cytosol. Meunier et al. explored light-indirect-responsive NBs for the delivery of pDNA into human cancer cells by femtosecond laser activation of AuNPs and found much higher transfection efficiency accompanied by very low toxicity compared to conventional lipofection. ${ }^{277}$ Similarly, Lukianova-Hleb et al. used a picosecond laser for high-throughput transfection of 'hard-to-transfect' T-cells with pDNA encoding green fluorescence protein. ${ }^{278}$ Xiong et al. compared the transfection efficiency of thermal induced cell membrane pores versus pores created by light-indirect-responsive NBs and found that NBmediated poration allowed more efficient entry of siRNA into cells. ${ }^{212}$ Recently, our group also explored light-indirect-responsive NBs for the intracellular delivery of siRNA and mRNA to human T cells for cellbased therapy. ${ }^{279,} 280$ In conclusion, light-indirect-responsive NBs may represent a promising method for the direct delivery of nucleic acids into the cell cytosol considering their high throughput, high 
efficacy and low cytotoxicity, although the above studies have mainly focused on in vitro or ex vivo-experimental models.

\subsubsection{Antibiotic delivery}

Direct- or indirect-responsive NBs have also been proposed as a promising strategy to overcome the current challenges related to increasing antibiotic resistance. The ultrasound-direct-responsive NBs loaded with the antibiotic vancomycin was reported by Argenziano et al. to be active against methicillin-resistant Staphylococcus aureus (MRSA) infections with improved oral bioavailability and formulation stability. ${ }^{206}$ The NBs presented good performance for prolonging antibiotic-release kinetics and inducing antibacterial activity, which was more sustained over time and more effective than vancomycin treatment alone in MRSA killing. Most importantly, the directly responsive NBs associated with ultrasound stimulation could promote antibiotic delivery in an in vitro model of porcine skin and improved antibacterial killing activity.

Light-indirect-responsive NBs have also been explored to improve penetration of antimicrobial agents through biofilms and to reduce antibiotic tolerance. ${ }^{281-283}$ As shown in Fig. 34, the proposed approach makes use of light-indirect-responsive NBs to locally disturb the integrity of the biofilm, potentiating antibiotic penetration, and substantially increasing its effectiveness. Specifically, biofilms are first treated with a suspension of AuNPs, which can gradually penetrate between sessile cells. A short $(<10$ nanoseconds) high-intensity laser pulse is used to irradiate such AuNPs in the biofilm to induce the indirect-responsive NBs (transient vapor NBs). The localized mechanical force caused by indirectresponsive NBs increases the space between cells, allowing antibiotics to easily reach the target cells, even those located deeply within the dense cell clusters. In addition, due to the fine control of laser light and localized action of indirect-responsive NBs, the biofilm disturbing effect can be very precisely controlled in space. The results showed that these light-indirect-responsive NBs lead to substantial local disruptions of biofilms in both Gram-negative (Burkholderia multivorans, Pseudomonas aeruginosa) and Gram-positive (Staphylococcus aureus) bacteria, and increases tobramycin efficacy by up to 1-3 orders of magnitude depending on the organism and treatment conditions.

\subsubsection{Delivery of therapeutic gases}

Administration of therapeutic gases like nitric oxide, hydrogen sulfide, carbon monoxide, and oxygen has attracted increasing interest as these gaseous molecules mediate numerous cell signaling pathways and exert important physiological roles, and have also been shown to evoke biological responses. ${ }^{284}$ Oxygen delivery is particularly interesting since many medical conditions, such as diabetes, burns, bedsores and other wounds, are associated with insufficient oxygen supply to tissues. ${ }^{285}$

The ultrasound-direct or indirect-responsive NBs have been proposed as a promising strategy for oxygen delivery. The ultrasound-direct-responsive NBs show good capacity to safely encapsulate and store oxygen. At the same time they do not cause hemolytic activity or toxic effects in cells. ${ }^{202,} 286$ Bhandari et al. showed that oxygen loaded NBs stimulated by ultrasound were efficacious for enhanced bladder cancer therapy. ${ }^{287}$ Ficiarà et al. demonstrated by MRI imaging that oxygen-loaded NBs labelled with SPIONs could cross the blood-brain-barrier upon ultrasound stimulation and could migrate to the central nervous system (CNS) to deliver and release oxygen. ${ }^{288}$ Recently, Song et al. reported a pHdirect-responsive NBs formulation loaded with oxygen used for spontaneous oxygen delivery in hypoxic tumors. ${ }^{153}$ They synthesized oxygen-loaded pH-responsive NBs shelled by acetalated dextran polymer to deliver spontaneous oxygenation in response to a minor $\mathrm{pH}$ drop in the tumor microenvironment. The oxygen delivery by these $\mathrm{pH}$-responsive NBs was evaluated in CNE2 tumor-bearing mice, and the intra-tumoral oxygen level increased by 6 -fold after the administration of the oxygen NBs without the need for any external triggering.

Conversely, ultrasound-indirect-responsive NBs have also been explored for oxygen delivery. ${ }^{289}$ Specifically, oxygen is loaded in chitosan-shelled nanodroplets composed of DFP. In solution, the gellike polymer structure can decrease gas diffusion, thus offering good oxygen carrying capacity, with no toxic effects on human keratinocytes. Upon applying an ultrasound trigger for NB formation from the nanodroplets a significant and time-sustained oxygen release in vivo can be achieved. Furthermore, the same group applied a similar system to deliver oxygen for treatment of skin infections. Oxygen was efficiently released by ultrasound stimulated nanodroplets to form indirect-responsive NBs, and displayed cytostatic activity against MRSA or Candida albicans but was not toxic to keratinocytes. ${ }^{290}$ 


\subsection{Ablation of tumor cells or biological tissues}

Ultrasound-direct-responsive NBs have been exploited for efficient ablation of tumor cells or biological tissues. Zhang et al. reported the synthesis of ultrasound-direct-responsive NBs for enhanced removal of residual tumor cells. ${ }^{200}$ They synthesized PLGA-shelled NBs to target tumor cells by conjugating monoclonal anti-HLA-G antibodies onto the surface of NBs. The results showed that the targeted NBs with high-intensity focused ultrasound (HIFU) stimulation significantly improved the efficiency of residual tumor cell ablation. Huang et al. used ultrasound-direct-responsive NBs to generate local and reversible blood-brain barrier disruption to deliver the therapeutic agents to the diseased areas of the brain for the treatment of brain tumors or diseases of the central CNS. ${ }^{208}$ Recently, similar results were also confirmed by another research group. ${ }^{291}$

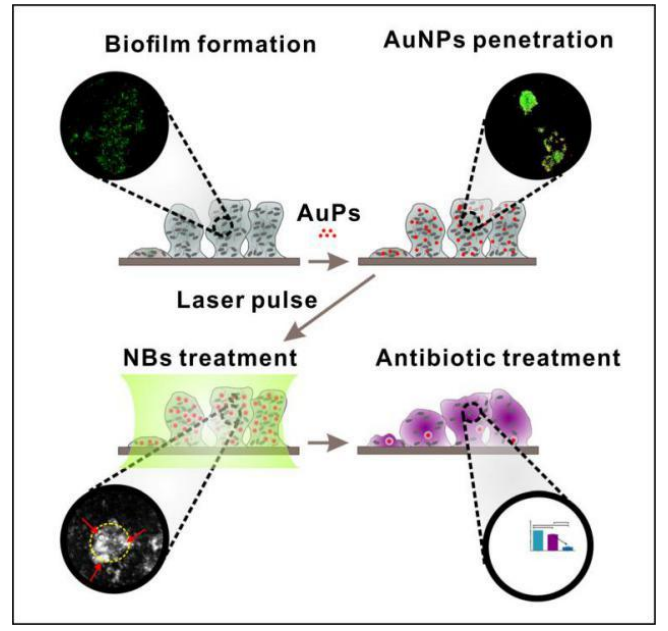

Fig. 34. Schematic overview of light-indirect-responsive NBs to improve penetration of antibiotics through biofilms for more effective biofilm eradication and reduction of antibiotic resistance. (a) Formation of a 24-h bacterial biofilm in vitro on a glass surface. (b) Penetration of AuNPs through the biofilm. (c) After absorption of an intense nanosecond laser pulse, NBs emerge around the AuNP. (d) The mechanical force of NBs creates more space between the cells allowing better penetration of subsequently administered antimicrobial agents. The figure is adapted from reference ${ }^{281}$

Conversely, light-indirect-responsive NBs have also been used to eliminate residual tumor cells during surgery. ${ }^{292}$ In mouse models, residual cancer cells were completely removed and local recurrence was prevented. The light-indirect-responsive NBs were also used for efficient and safe ablation of floaters, which are formed by collagen aggregation upon aging in the vitreous body of the human eye, or by acute posterior vitreous detachment. The vitreous floaters can severely disturb vision due to light scattering by the dense collagen matrix. Recently, light-indirect-responsive NBs were generated by pulsed-laser illumination of AuNPs to ablate vitreous floaters. ${ }^{293}$
After binding of the AuNPs to the collagen aggregates, the collagen aggregates were efficiently destroyed using approximately 1000 times less light energy than that typically used in neodymium-doped yttrium aluminum garnet (Nd:YAG) laser therapy, which substantially increases the safety of the procedure.

Kosar et al. found that hydrodynamic-responsive NBs could induce damage on leukemia/lymphoma cells, kill prostate cells, and ablate benign prostatic hyperplasia tissue. ${ }^{294}, 295$ The same group also demonstrated the potential of these collapsible bubbles for use in kidney stone treatment in vitro and found that they could successfully erode stones with an erosion rate of $0.31 \mathrm{mg} / \mathrm{min} .{ }^{296}$ Subsequently, they also developed a biomedical device prototype based on microscale hydrodynamic cavitation to demonstrate the feasibility of an alternative treatment for urinary stone therapy and abnormal tissue ablation. ${ }^{297}$

\section{Conclusions and future perspectives}

NBs offer many advantages for a wide range of applications in a variety of fields, including biomedicine, environmental applications, and agriculture. The advantages of NBs include their excellent and unique properties like small size, high surface-to- volume ratio, and high surface adsorption capacity. In particular, stimuli-responsive NBs are of considerable interest in biomedical applications as they can be activated in a spatiotemporally resolved manner in response to external or internal stimuli. Quite advanced biomedical applications are possible, such as multimodal imaging, molecularly-targeted imaging, or targeted drug delivery.

In our opinion, the following research directions should be further explored in the future.

(a) There are numerous reports on the theory of NBs, especially related to their surface properties. However, additional theoretical research is needed on the mechanisms involved in the response of NBs to external triggers. Inspiration may be found in related research on macro- and microbubbles, the biophysics of which has already been studies thoroughly. ${ }^{298}$ In future research it will be of interest to validate to which extent these theories are suited or need adaptations to describe the behavior of stimuli responsive NBs. Such fundamental knowledge will improve our insights into the behavior of NBs under 
triggering stimuli and may further improve the design of stimuli-responsive NBs for specific applications.

(b) Currently, most research on stimuli-responsive NBs have focused on exogenous stimuli, especially ultrasound and light. Instead, only a few publications have described $\mathrm{pH}$ responsive NBs as a form of endogenous stimuli-responsive NBs. Thus, there is ample room for developing additional types of endogenous stimuli-responsive NBs to expand their application in biomedicine. An interesting endogenous stimulus to be exploited is enzymatic activity, for instance to achieve controlled drug delivery to the microenvironment of tumors or biofilms, which display differential expression of specific enzymes compared to normal tissues. ${ }^{299}$

(c) Physical or chemical methods have been used extensively to synthesize stimuli-responsive NBs. However, studies evaluating biological methods for the generation of stimuliresponsive NBs are limited. Indeed, only a few reports on a type of gas vesicles have been published recently. ${ }^{300-302}$ These vesicles are gas-filled protein-shelled compartments in cylindrical or biconical shapes with typical widths of 45$250 \mathrm{~nm}$ and lengths of 100-600 $\mathrm{nm}$ that exclude water and are permeable to gas. They are generated by genetically encoded gas nanostructures formed by certain bacteria and archaea as a means to control buoyancy for optimal access to light and nutrients. Most importantly, preliminary results have already demonstrated these gas vesicles share the unique intrinsic properties of NBs, such as responsiveness to ultrasound stimuli. Unlike NBs formed by physical or chemical methods, the protein shells of gas vesicles exclude water but are freely permeable to gases in the surrounding media. ${ }^{303}$

In conclusion, stimuli-responsive NBs have become interesting and promising biomedical nanotools, which may introduce a new era of scientific advancement to the service of humans due to their excellent and unique features and the vast array of potential applications.

\section{Conflicts of interest}

There are no conflicts to declare.

\section{Acknowledgements}

This research was supported by National Natural Science Foundation of China (No. 21774060 and No. 21644004), the European Research Council (ERC Consolidator Grant, 648124), and the Research Foundation Flanders (FWO, 1500418N and $12 \mathrm{Q} 8718 \mathrm{~N})$. The authors would like to acknowledge funding from the European Union's Horizon 2020 Research and Innovation Programme under grant agreement No. 810685 (DelNam project).

\section{References}

1. H. Tsuge, Micro- and Nanobubbles: Fundamentals and Applications, Jenny Stanford Publishing, 2014.

2. A. S. Najafi, J. Drelich, A. Yeung, Z. Xu and J. Masliyah, Journal of Colloid and Interface Science, 2007, 308, 344350.

3. X. H. Zhang, X. D. Zhang, J. L. Sun, Z. X. Zhang, G. Li, H. P. Fang, X. D. Xiao, X. C. Zeng and J. Hu, Langmuir, 2007, 23, 1778-1783.

4. M. Guan, W. Guo, L. H. Gao, Y. Z. Tang, J. Hu and Y. M. Dong, Chemphyschem, 2012, 13, 2115-2118.

5. S. S. Kistler, Nature, 1931, 127, 741-741.

6. Z. H. Wu, H. B. Chen, Y. M. Dong, H. L. Mao, J. L. Sun, S. F. Chen, V. S. J. Craig and J. Hu, Journal of Colloid and Interface Science, 2008, 328, 10-14

$7 . \quad J$. Zhu, H. J. An, M. Alheshibri, L. D. Liu, P. M. J. Terpstra, G. M. Liu and V. S. J. Craig, Langmuir, 2016, 32, 11203-11211.

8. A. J. Atkinson, O. G. Apul, O. Schneider, S. Garcia-Segura and P. Westerhoff, Accounts Chem Res, 2019, 52, 11961205.

9. K. Ebina, K. Shi, M. Hirao, J. Hashimoto, Y. Kawato, S. Kaneshiro, T. Morimoto, K. Koizumi and H. Yoshikawa, Plos One, 2013, 8.

10. S. Liu, S. Oshita, Y. Makino, Q. H. Wang, Y. Kawagoe and T. Uchida, Acs Sustain Chem Eng, 2016, 4, 1347-1353.

11. P. M. Sharif, A. A. Hairuddin, A. As'arry, K. A. M. Rezali, M. M. Noor, M. Norhafana and S. M. Shareef, Iop Conf Ser-Mat Sci, 2019, 469.

12. A. Ushida, T. Hasegawa, T. Nakajima, H. Uchiyama and T. Narumi, Exp Therm Fluid Sci, 2012, 39, 54-59.

13. H. Sayyaadi and M. Nematollahi, Scientia Iranica, 2013, 20, 535-541.

14. A. Hashim, O. Yaakob, K. Koh, N. Ismail and Y. Ahmed, Jurnal Teknologi, 2015, 74, 105-114.

15. R. Cavalli, M. Soster and M. Argenziano, Ther Deliv, 2016, 7, 117-138.

16. A. T. Ayodele, A. Valizadeh, M. Adabi, S. S. Esnaashari, F. Madani, M. Khosravani and M. Adabi, Biointerface Res App, 2017, 7, 2253-2262.

17. D. Lohse, Phys Rev Fluids, 2018, 3.

18. J. R. T. Seddon, D. Lohse, W. A. Ducker and V. S. J. Craig Chemphyschem, 2012, 13, 2179-2187.

19. D. Lohse and X. H. Zhang, Rev Mod Phys, 2015, 87, 9811035. 
20

M. Alheshibri, J. Qian, M. Jehannin and V. S. J. Craig, Langmuir, 2016, 32, 11086-11100.

21. L. E. Andrews, M. H. Chan and R. S. Liu, Nanotechnology, 2019, 30.

22.

P. E. Theodoraki and Z. Z. Che, Adv Colloid Interfac, 2019, 272.

23

S. T. Lou, Z. Q. Ouyang, Y. Zhang, X. J. Li, J. Hu, M. Q. Li and F. J. Yang, J Vac Sci Technol B, 2000, 18, 2573-2575.

N. Ishida, T. Inoue, M. Miyahara and K. Higashitani, Langmuir, 2000, 16, 6377-6380.

25.

N. K. Dube, B. E. Oeffinger and M. A. Wheatley, Proceedings of the leee 29th Annual Northeast Bioengineering Conference, 2003, 102-103.

26.

B. E. Oeffinger and M. A. Wheatley, Ultrasonics, 2004, 42, 343-347.

27.

D. Lapotko, Nanomedicine-Uk, 2009, 4, 813-845.

28.

29.

30.

31.

32.

33.

34.

35

36.

37.

38

39.

40.

41.

42.

43

44

45

46

47.

48.

49.

50
V. B. Svetovoy, R. G. P. Sanders and M. C. Elwenspoek, J Phys-Condens Mat, 2013, 25.

C. E. Brennen, Interface Focus, 2015, 5.

E. Badfar and M. A. Ardestani, Sn Appl Sci, 2019, 1.

J. Y. Kim, M. G. Song and J. D. Kim, Journal of Colloid and Interface Science, 2000, 223, 285-291.

D. Lapotko, Int J Heat Mass Tran, 2009, 52, 1540-1543.

V. B. Svetovoy, R. G. P. Sanders, T. S. J. Lammerink and M.

C. Elwenspoek, Phys Rev E, 2011, 84.

O. Neumann, A. S. Urban, J. Day, S. Lal, P. Nordlander and N. J. Halas, Acs Nano, 2013, 7, 42-49.

P. Riesz, D. Berdahl and C. L. Christman, Environ Health Persp, 1985, 64, 233-252.

A. Sarc, J. Kosel, D. Stopar, M. Oder and M. Dular, Ultrason Sonochem, 2018, 42, 228-236.

F. Sauvage, J. C. Fraire, K. Remaut, J. Sebag, K. Peynshaert, M. Harrington, F. J. Van de Velde, R. H. Xiong, M. J. Tassignon, T. Brans, K. Braeckmans and S. C. De Smedt, Acs Nano, 2019, 13, 8401-8416.

M. A. Hampton and A. V. Nguyen, Adv Colloid Interfac, 2010, 154, 30-55.

J. R. T. Seddon and D. Lohse, J Phys-Condens Mat, 2011, 23.

H. Lhuissier, D. Lohse and X. H. Zhang, Soft Matter, 2014, 10, 942-946.

Y. W. Liu and X. R. Zhang, Chinese Phys B, 2018, 27.

A. Agarwal, W. J. Ng and Y. Liu, Chemosphere, 2011, 84, $1175-1180$

X. H. Zhang, A. Kumar and P. J. Scales, Langmuir, 2011, 27, 2484-2491.

S. Calgaroto, K. Q. Wilberg and J. Rubio, Miner Eng, 2014,

S. H. Oh, S. H. Yoon, H. Song, J. G. Han and J. M. Kim, Int J Hydrogen Energ, 2013, 38, 14849-14853.

J. G. Fujimoto, W. Z. Lin, E. P. Ippen, C. A. Puliafito and R. F. Steinert, Investigative Ophthalmology \& Visual Science, 1985, 26, 1771-1777.

R. Kattan, A. Denat and O. Lesaint, J Appl Phys, 1989, 66, 4062-4066.

C. K. Holland and R. E. Apfel, J Acoust Soc Am, 1990, 88, 2059-2069.

A. Vogel, J. Noack, G. Huttman and G. Paltauf, Appl Phys BLasers O, 2005, 81, 1015-1047.

R. H. Xiong, S. K. Samal, J. Demeester, A. G. Skirtach, S. C. De Smedt and K. Braeckmans, Adv Phys-X, 2016, 1, 596620. 60, 33-40.
51.

53.

54.

55.

56.

57.

58.

59.

60.

61.

62.

63.

66.

67.

69.

70.

71.

72.

73.

74.

75.

76.

77.

78.

79.
E. Y. Lukianova-Hleb, K. M. Campbell, P. E. Constantinou, J. Braam, J. S. Olson, R. E. Ware, D. J. Sullivan and D. O. Lapotko, P Natl Acad Sci USA, 2014, 111, 900-905.

P. R. Birkin, S. Linfield, J. J. Youngs and G. Denuault, J Phys Chem C, 2020, 124, 7544-7549.

International Pat., 2010.

US Pat., 2012.

E. Svenson and J. Willits, in Green Vegetable Oil Processing, eds. W. E. Farr and A. Proctor, AOCS Press, 2014, DOI: https://doi.org/10.1016/B978-0-9888565-3-0.50010-8, pp. 147-157.

A. M. Fales, W. C. Vogt, K. A. Wear, T. J. Pfefer and I. K. llev, J Biomed Opt, 2019, 24, 1-10.

M. Versluis, E. Stride, G. Lajoinie, B. Dollet and T. Segers, Ultrasound Med Biol, 2020, 46, 2117-2144.

M. Ashokkumar, Ultrason Sonochem, 2011, 18, 864-872.

I. Lentacker, I. De Cock, R. Deckers, S. C. De Smedt and C. T. W. Moonen, Adv Drug Deliver Rev, 2014, 72, 49-64.

F. Jin, J. Ye, L. Hong, H. Lam and C. Wu, J Phys Chem B, 2007, 111, 2255-2261.

W. A. Ducker, Langmuir : the ACS journal of surfaces and colloids, 2009, 25, 8907-8910.

R. Cavalli, A. Bisazza, M. Trotta, M. Argenziano, A. Civra, M. Donalisio and D. Lembo, Int J Nanomed, 2012, 7, 33093318.

X. M. Zhang, Y. Y. Zheng, Z. G. Wang, S. Huang, Y. Chen, W. Jiang, H. Zhang, M. X. Ding, Q. S. Li, X. Q. Xiao, X. Luo, Z. B. Wang and H. B. Qi, Biomaterials, 2014, 35, 5148-5161.

R. Cavalli, M. Argenziano, E. Vigna, P. Giustetto, E. Torres, S. Aime and E. Terreno, Colloid Surface B, 2015, 129, 39-46. M. Lafond, A. Watanabe, S. Yoshizawa, S.-i. Umemura and K. Tachibana, Sci Rep-Uk, 2018, 8, 7472.

F. Bessone, M. Argenziano, G. Grillo, B. Ferrara, S. Pizzimenti, G. Barrera, G. Cravotto, C. Guiot, I. Stura, R. Cavalli and C. Dianzani, Nanotechnology, 2019, 30.

H. Kida, K. Nishimura, K. Ogawa, A. Watanabe, L. B. Feril, Y. Irie, H. Endo, S. Kawakami and K. Tachibana, Front Pharmacol, 2020, 11.

R. Diaz-Lopez, N. Tsapis, M. Santin, S. L. Bridal, V. Nicolas, D. Jaillard, D. Libong, P. Chaminade, V. Marsaud, C. Vauthier and E. Fattal, Biomaterials, 2010, 31, 1723-1731. C. Hernandez, L. Nieves, A. de Leon, R. Advincula and A. A. Exner, Acs Appl Mater Inter, 2018, 10, 9949-9956.

C. R. Hill, J Acoust Soc Am, 1972, 52, 667-\&.

H. Medwin, J Acoust Soc Am, 1977, 62, 1041-1044.

D. G. Shchukin, E. Skorb, V. Belova and H. Mohwald, Adv Mater, 2011, 23, 1922-1934.

V. Belova, M. Krasowska, D. Y. Wang, J. Ralston, D. G. Shchukin and H. Mohwald, Chem Sci, 2013, 4, 248-256.

J. J. Kwan, S. Graham, R. Myers, R. Carlisle, E. Stride and C. C. Coussios, Phys Rev E, 2015, 92. 2019, 35, 10106-10115.

Q. F. Jin, C. Y. Lin, S. T. Kang, Y. C. Chang, H. R. Zheng, C. M. Yang and C. K. Yeh, Ultrason Sonochem, 2017, 36, 262-269. A. Sviridov, K. Tamarov, I. Fesenko, W. Xu, V. Andreev, V. Timoshenko and V.-P. Lehto, Frontiers in Chemistry, 2019, 7.

O. D. Kripfgans, J. B. Fowlkes, D. L. Miller, O. P. Eldevik and P. L. Carson, Ultrasound Med Biol, 2000, 26, 1177-1189.

N. Y. Rapoport, A. M. Kennedy, J. E. Shea, C. L. Scaife and K. H. Nam, J Control Release, 2009, 138, 268-276. 
80

P. S. Sheeran, V. P. Wong, S. Luois, R. J. McFarland, W. D. Ross, S. Feingold, T. O. Matsunaga and P. A. Dayton, Ultrasound Med Biol, 2011, 37, 1518-1530.

81. P. S. Sheeran and P. A. Dayton, Curr Pharm Design, 2012, 18, 2152-2165.

82. P. S. Sheeran and P. A. Dayton, Scientifica, 2014, 2014, 579684.

83. P. Bhandari, X. Wang and J. Irudayaraj, Acs Nano, 2017, 11 2682-2688.

84. Z. Fang, Y.-R. Zhen, O. Neumann, A. Polman, F. J. García de Abajo, P. Nordlander and N. J. Halas, Nano Letters, 2013, 13, 1736-1742.

85 Y. S. Pang, J. J. Zhang, R. M. Ma, Z. G. Qu, E. Lee and T. F. Luo, Acs Energy Lett, 2020, 5, 437-456.

86. Q. Wang, H. Zhao, N. Qi, Y. Qin, X. Zhang and Y. Li, Sci RepUk, 2019, 9, 1118.

87. L. Hou, M. Yorulmaz, N. R. Verhart and M. Orrit, New J Phys, 2015, 17, 013050.

88. S. Maheshwari, M. van der Hoef, A. Prosperetti and D. Lohse, J Phys Chem C, 2018, 122, 20571-20580.

89. S. Merabia, S. Shenogin, L. Joly, P. Keblinski and J. L. Barrat, P Natl Acad Sci USA, 2009, 106, 15113-15118.

90.

A. Siems, S. A. L. Weber, J. Boneberg and A. Plech, New J Phys, 2011, 13.

91. E. Lukianova-Hleb, Y. Hu, L. Latterini, L. Tarpani, S. Lee, R. A. Drezek, J. H. Hafner and D. O. Lapotko, Acs Nano, 2010, 4, 2109-2123.

92. T. Katayama, K. Setoura, D. Werner, H. Miyasaka and S. Hashimoto, Langmuir, 2014, 30, 9504-9513.

93. E. Y. Lukianova-Hleb, A. N. Volkov and D. O. Lapotko, Langmuir, 2014, 30, 7425-7434.

94.

K. Metwally, S. Mensah and G. Baffou, The Journal of Physical Chemistry C, 2015, 119, 28586-28596.

95. D. Lapotko, Opt Express, 2009, 17, 2538-2556.

96. E. Y. Lukianova-Hleb, M. B. Mutonga and D. O. Lapotko, Acs Nano, 2012, 6, 10973-10981.

97. E. Y. Lukianova-Hleb, D. S. Wagner, M. K. Brenner and D. O. Lapotko, Biomaterials, 2012, 33, 5441-5450.

98. M. O. Ogunyankin, J. E. Shin, D. O. Lapotko, V. E. Ferry and J. A. Zasadzinski, Adv Funct Mater, 2018, 28

99. J. E. Shin, M. O. Ogunyankin and J. A. Zasadzinski, Small, 2019, 15, 1804476.

100. J. Lombard, T. Biben and S. Merabia, Phys Rev Lett, 2014, 112, 105701

101. M. E. Zaytsev, Y. Wang, Y. Zhang, G. Lajoinie, X. Zhang, A Prosperetti, H. J. W. Zandvliet and D. Lohse, The Journal of Physical Chemistry C, 2020, 124, 5861-5869.

102. T. Nakajima, X. Wang, S. Chatterjee and T. Sakka, Sci RepUk, 2016, 6, 28667.

103. A. Dagallier, E. Boulais, C. Boutopoulos, R. Lachaine and M. Meunier, Nanoscale, 2017, 9, 3023-3032.

104. J. Baumgart, L. Humbert, É. Boulais, R. Lachaine, J. J. Lebrun and M. Meunier, Biomaterials, 2012, 33, 2345-2350.

105. E. Boulais, R. Lachaine and M. Meunier, Nano letters, 2012 12, 4763-4769.

106. R. Lachaine, E. Boulais and M. Meunier, Acs Photonics, 2014, 1, 331-336.

107. K. R. Rau, P. A. Quinto-Su, A. N. Hellman and V. Venugopalan, Biophys J, 2006, 91, 317-329.

108. J. Lam, J. Lombard, C. Dujardin, G. Ledoux, S. Merabia and D. Amans, Applied Physics Letters, 2016, 108, 074104.
109. A. Vogel, N. Linz, S. Freidank and G. Paltauf, Phys Rev Lett, 2008, 100, 038102

$110 . \quad$ B. Y. Zhao, Y. Song, S. Wang, B. Dai, L. J. Zhang, Y. M. Dong, J. H. Lu and J. Hu, Soft Matter, 2013, 9, 8837-8843.

111. W. Walczyk, P. M. Schon and H. Schonherr, J Phys-Condens Mat, 2013, 25.

112. W. Walczyk and H. Schonherr, Langmuir, 2014, 30, 1195511965.

113. H. An, B. H. Tan and C.-D. Ohl, Langmuir, 2016, 32, 1271012715.

114. W. Walczyk, N. Hain and H. Schönherr, Soft Matter, 2014 10, 5945-5954.

115. M. Vedadi, A. Choubey, K. Nomura, R. K. Kalia, A. Nakano, P. Vashishta and A. C. T. van Duin, Phys Rev Lett, 2010, 105, 014503.

116. A. Shekhar, K.-i. Nomura, R. K. Kalia, A. Nakano and P. Vashishta, Phys Rev Lett, 2013, 111, 184503.

117. U. Adhikari, A. Goliaei and M. L. Berkowitz, The Journal of Physical Chemistry B, 2015, 119, 6225-6234.

118. A. Goliaei, U. Adhikari and M. L. Berkowitz, ACS Chemical Neuroscience, 2015, 6, 1296-1301.

119. Q. Hu, L. Zhang and R. P. Joshi, AIP Advances, 2019, 9, 045006.

120. J. Carpenter, M. Badve, S. Rajoriya, S. George, V. Saharan and A. Pandit, Reviews in Chemical Engineering, 2016, 33. X. H. Zhang, G. Li, Z. H. Wu, X. D. Zhang and J. Hu, Chinese Phys, 2005, 14, 1774-1778.

122.

R. P. Berkelaar, J. R. T. Seddon, H. J. W. Zandvliet and D. Lohse, Chemphyschem, 2012, 13, 2213-2217.

R. Berkelaar, J. Seddon, H. Zandvliet and D. Lohse, Chemphyschem : a European journal of chemical physics and physical chemistry, 2012, 13, 2213-2217.

124. R. Ahmadi and A. Khodadadi Darban, International Journal of Nanoscience and Nanotechnology, 2013, 9, 151-162.

N. Nirmalkar, A. W. Pacek and M. Barigou, Langmuir, 2018, 34, 10964-10973.

N. Nirmalkar, A. W. Pacek and M. Barigou, Soft Matter, 2018, 14, 9643-9656.

B. Bhushan, Y. Pan and S. Daniels, Journal of Colloid and Interface Science, 2013, 392, 105-116.

L. Wu, Y. Han, Q. Zhang and S. Zhao, RSC Advances, 2019, 9, 1792-1798.

L. J. Zhang, Y. Zhang, X. H. Zhang, Z. X. Li, G. X. Shen, M. Ye, C. H. Fan, H. P. Fang and J. Hu, Langmuir, 2006, 22, 81098113.

130. L. Luo and H. S. White, Langmuir, 2013, 29, 11169-11175.

131. Q. Chen, H. S. Wiedenroth, S. R. German and H. S. White, Journal of the American Chemical Society, 2015, 137, 12064-12069.

132.

S. R. German, M. A. Edwards, Q. Chen and H. S. White, Nano Letters, 2016, 16, 6691-6694.

133. X. Zhao, H. Ren and L. Luo, Langmuir, 2019, 35, 5392-5408.

134. Y. A. Perez Sirkin, E. D. Gadea, D. A. Scherlis and V. Molinero, Journal of the American Chemical Society, 2019, 141, 10801-10811.

135. M. R. Ghaani, P. G. Kusalik and N. J. English, Science Advances, 2020, 6, eaaz0094.

V. B. Svetovoy, R. G. P. Sanders, T. S. J. Lammerink and M. C. Elwenspoek, Phys Rev E, 2011, 84, 035302.

V. B. Svetovoy, R. G. Sanders and M. C. Elwenspoek, J Phys Condens Matter, 2013, 25, 184002. 
138. V. B. Svetovoy, R. G. P. Sanders, K. Ma and M. C. Elwenspoek, Sci Rep-Uk, 2014, 4, 4296.

139. A. V. Postnikov, I. V. Uvarov, M. V. Lokhanin and V. B. Svetovoy, Plos One, 2017, 12.

140. H.-Y. Huang, H.-L. Liu, P.-H. Hsu, C.-S. Chiang, C.-H. Tsai, H.S. Chi, S.-Y. Chen and Y.-Y. Chen, Adv Mater, 2015, 27, 655661.

141. W. Song, Y. Luo, Y. Zhao, X. Liu, J. Zhao, J. Luo, Q. Zhang, H. Ran, Z. Wang and D. Guo, Nanomedicine-Uk, 2017, 12, 9911009.

142. T. Li, J. Zhou, C. Zhang, X. Zhi, J. Niu, H. Fu, J. Song and D. Cui, NPG Asia Materials, 2018, 10, 1046-1060.

143. P. Vallée, J. Lafait, L. Legrand, P. Mentré, M.-O. Monod and Y. Thomas, Langmuir, 2005, 21, 2293-2299.

144. K. Uehara and Y. Yano, IEEE Transactions on Magnetics, 2011, 47, 2604-2607.

145. J.-Y. Kim, M.-G. Song and J.-D. Kim, Journal of Colloid and Interface Science, 2000, 223, 285-291.

146. M. Takahashi, The Journal of Physical Chemistry B, 2005, 109, 21858-21864.

147. A. Gray-Weale and J. K. Beattie, Phys Chem Chem Phys, 2009, 11, 10994-11005.

148. S. Uddin, L. Jin, M. Mirnezami and J. A. Finch, Journal of Colloid and Interface Science, 2013, 389, 298-305.

149. W. Jia, S. Ren and B. Hu, International Journal of Electrochemical Science, 2013, 8, 5828-5837.

150. S.-H. Cho, J.-Y. Kim, J.-H. Chun and J.-D. Kim, Colloids and Surfaces A: Physicochemical and Engineering Aspects, 2005, 269, 28-34.

151. E. M. Bachelder, T. T. Beaudette, K. E. Broaders, J. Dashe and J. M. J. Fréchet, Journal of the American Chemical Society, 2008, 130, 10494-10495.

152. K. E. Broaders, J. A. Cohen, T. T. Beaudette, E. M. Bachelder and J. M. J. Fréchet, Proceedings of the National Academy of Sciences, 2009, 106, 5497-5502.

153. R. Song, S. Peng, Q. Lin, M. Luo, H. Y. Chung, Y. Zhang and S. Yao, Langmuir, 2019, 35, 10166-10172.

154. C. J. Ke, W. L. Chiang, Z. X. Liao, H. L. Chen, P. S. Lai, J. S. Sun and H. W. Sung, Biomaterials, 2013, 34, 1-10.

155. Q. Liu, X. Chen, J. Jia, W. Zhang, T. Yang, L. Wang and G. Ma, Acs Nano, 2015, 9, 4925-4938.

156. E. Y. Lukianova-Hleb, K. M. Campbell, P. E. Constantinou, J. Braam, J. S. Olson, R. E. Ware, D. J. Sullivan and D. O. Lapotko, Proceedings of the National Academy of Sciences, 2013, DOI: 10.1073/pnas.1316253111, 201316253.

157. Ş. Hamarat Şanlıer, G. Ak, H. Yılmaz, A. Ünal, Ü. F. Bozkaya, G. Tanıyan, Y. Yıldırım and G. Yıldız Türkyılmaz, Journal of Pharmaceutical Sciences, 2019, 108, 1272-1283.

158. B. Luo, H. Zhang, X. Liu, R. Rao, Y. Wu and W. Liu, Biomed Mater Eng, 2015, 26 Suppl 1, S911-916.

159. Y. X. Wang, M. M. Lan, D. J. Shen, K. J. Fang, L. H. Zhu, Y. Liu, L. Hao and P. Li, Int J Nanomed, 2020, 15, 4289-4309.

160. B. E. Oeffinger and M. A. Wheatley, Ultrasonics, 2004, 42, 343-347.

161. K. Yasuda, H. Matsushima and Y. Asakura, Chem Eng Sci, 2018, 195.

162. United States Pat., 2007.

163. EUROPEAN Pat., EP 3124109 A1, 2017.

164. F. Y. Ushikubo, T. Furukawa, R. Nakagawa, M. Enari, Y. Makino, Y. Kawagoe, T. Shiina and S. Oshita, Colloids and Surfaces A: Physicochemical and Engineering Aspects, 2010, 361, 31-37.
165.

166.

Z. Fang, L. Wang, X. Wang, L. Zhou, S. Wang, Z. Zou, R. Tai, L. Zhang and J. Hu, The Journal of Physical Chemistry C, 2018, 122, 22418-22423.

J66. Jin, Z. Feng, F. Yang and N. Gu, Langmuir, 2019, 35, 42384245.

167. S. Ke, W. Xiao, N. Quan, Y. Dong, L. Zhang and J. Hu, Langmuir, 2019, 35, 5250-5256.

168. S. Maeda, H. Kobayashi, K. Ida, M. Kashiwa, I. Nishihara and T. Fujita, Proc Spie, 2014, 9232.

169. Z. Pourkarimi, B. Rezai and M. Noaparast, Physicochem Probl Mi, 2017, 53, 920-942.

170. Z. Pourkarimi, B. Rezai and M. Noaparast, Fizykochemiczne Problemy Mineralurgii - Physicochemical Problems of Mineral Processing, 2017, 54.

171. H. Oliveira, A. Azevedo and J. Rubio, Miner Eng, 2018, 116, 32-34.

172.

E. D. Michailidi, G. Bomis, A. Varoutoglou, G. Z. Kyzas, G. Mitrikas, A. C. Mitropoulos, E. K. Efthimiadou and E. P. Favvas, Journal of Colloid and Interface Science, 2020, 564, 371-380.

173. N. M. B. Smeets and T. F. L. McKenna, Journal of Colloid and Interface Science, 2012, 383, 28-35.

S. H. Oh and J.-M. Kim, Langmuir, 2017, 33, 3818-3823.

174.

175.

176.

M. Jannesari, O. Akhavan and H. R. Madaah Hosseini, Carbon, 2018, 138, 8-17.

C. Wu, K. Nesset, J. Masliyah and Z. Xu, Adv Colloid Interfac, 2012, 179-182, 123-132.

177. United States Pat., US 2016/0236158A1, 2016

178. International Pat., WO 2018/064689 Al, 2018.

179. M. Kukizaki and M. Goto, Journal of Membrane Science, 2006, 281, 386-396.

180. United States Pat., US 2012/0086137 A1, 2012.

181. United States Pat., USOO8678354B2, 2014.

182. EUROPEAN Pat., EP 2995369 A1, 2016.

183. J. Qiu, Z. Zou, S. Wang, X. Wang, L. Wang, Y. Dong, H. Zhao, L. Zhang and J. Hu, Chemphyschem, 2017, 18, 1345-1350.

184. J. C. Millare and B. A. Basilia, ChemistrySelect, 2018, 3, 9268-9275.

185. C. Sun, World journal of Pharmacy and pharmaceutical sciences, 2017, 1290-1314.

186. T. M. Krupka, L. Solorio, R. E. Wilson, H. Wu, N. Azar and A. A. Exner, Mol Pharmaceut, 2010, 7, 49-59.

187. J. Liu, B. Zhang, M. T. Li, M. J. Zhou, F. Li, X. X. Huang, M. Pan, L. Xue and F. Yan, Plos One, 2017, 12.

188. C. Hernandez, S. Gulati, G. Fioravanti, P. L. Stewart and A. A. Exner, Sci Rep-Uk, 2017, 7, 13517.

189. Z. Xing, J. Wang, H. Ke, B. Zhao, X. Yue, Z. Dai and J. Liu, Nanotechnology, 2010, 21, 145607.

190. W. B. Cai, H. L. Yang, J. Zhang, J. K. Yin, Y. L. Yang, L. J. Yuan, L. Zhang and Y. Y. Duan, Sci Rep-Uk, 2015, 5, 13725.

191. T. Yin, P. Wang, R. Zheng, B. Zheng, D. Cheng, X. Zhang and X. Shuai, Int J Nanomed, 2012, 7, 895-904.

192. J. Zhang, Y. Chen, C. Deng, L. Zhang, Z. Sun, J. Wang, Y. Yang, Q. Lv, W. Han and M. Xie, Front Pharmacol, 2019, 10. S. A. Peyman, J. R. McLaughlan, R. H. Abou-Saleh, G. Marston, B. R. G. Johnson, S. Freear, P. L. Coletta, A. F. Markham and S. D. Evans, Lab on a Chip, 2016, 16, 679-687.

194. B. Geers, H. Dewitte, S. C. De Smedt and I. Lentacker, Journal of controlled release : official journal of the Controlled Release Society, 2012, 164, 248-255.

195. N. Nomikou, P. Tiwari, T. Trehan, K. Gulati and A. P. McHale, Acta Biomater, 2012, 8, 1273-1280. 
196. J. P. Wang, J. P. Yan, J. Xu, T. H. Yin, R. Q. Zheng and W. Wang, Cancer Manag Res, 2019, 11, 6637-6649.

197. A. Prabhakar and R. Banerjee, ACS Omega, 2019, 4, 1556715580.

198. J. S. Xu, J. Huang, R. Qin, G. H. Hinkle, S. P. Povoski, E. W. Martin and R. X. Xu, Biomaterials, 2010, 31, 1716-1722.

199. C. Niu, Z. Wang, G. Lu, T. M. Krupka, Y. Sun, Y. You, W. Song, H. Ran, P. Li and Y. Zheng, Biomaterials, 2013, 34, 23072317.

200. X. Zhang, Y. Zheng, Z. Wang, S. Huang, Y. Chen, W. Jiang, H. Zhang, M. Ding, Q. Li, X. Xiao, X. Luo, Z. Wang and H. Qi, Biomaterials, 2014, 35, 5148-5161.

201. N. Rapoport, Z. Gao and A. Kennedy, JNCl: Journal of the National Cancer Institute, 2007, 99, 1095-1106.

202. R. Cavalli, A. Bisazza, P. Giustetto, A. Civra, D. Lembo, G. Trotta, C. Guiot and M. Trotta, Int J Pharm, 2009, 381, 160165.

203. R. Cavalli, A. Bisazza, M. Trotta, M. Argenziano, A. Civra, M. Donalisio and D. Lembo, Int J Nanomed, 2012, 7, 33093318.

204. R. Cavalli, M. Argenziano, E. Vigna, P. Giustetto, E. Torres, S. Aime and E. Terreno, Colloids Surf B Biointerfaces, 2015, 129, 39-46.

205. F. Marano, M. Argenziano, R. Frairia, A. Adamini, O. Bosco, L. Rinella, N. Fortunati, R. Cavalli and M. G. Catalano, Thyroid, 2016, 26, 705-716.

206. M. Argenziano, G. Banche, A. Luganini, N. Finesso, V. Allizond, G. R. Gulino, A. Khadjavi, R. Spagnolo, V. Tullio, G. Giribaldi, C. Guiot, A. M. Cuffini, M. Prato and R. Cavalli, Int J Pharm, 2017, 523, 176-188.

207. F. Bessone, M. Argenziano, G. Grillo, B. Ferrara, S. Pizzimenti, G. Barrera, G. Cravotto, C. Guiot, I. Stura, R. Cavalli and C. Dianzani, Nanotechnology, 2019, 30, 214004.

208. H. Y. Huang, H. L. Liu, P. H. Hsu, C. S. Chiang, C. H. Tsai, H. S. Chi, S. Y. Chen and Y. Y. Chen, Advanced materials (Deerfield Beach, Fla.), 2015, 27, 655-661.

209. R. Abdalkader, S. Kawakami, J. Unga, Y. Higuchi, R. Suzuki, K. Maruyama, F. Yamashita and M. Hashida, Drug Deliv, 2017, 24, 320-327.

210. J. Zhang, Y. Chen, C. Deng, L. Zhang, Z. Sun, J. Wang, Y. Yang, Q. Lv, W. Han and M. Xie, Front Pharmacol, 2019, 10, 610.

211. D. Midtvedt, F. Eklund, E. Olsén, B. Midtvedt, J. Swenson and F. Höök, Analytical Chemistry, 2020, 92, 1908-1915.

212. R. Xiong, K. Raemdonck, K. Peynshaert, I. Lentacker, I. De Cock, J. Demeester, S. C. De Smedt, A. G. Skirtach and K. Braeckmans, Acs Nano, 2014, 8, 6288-6296.

213. M. lijima, N. Gombodorj, Y. Tachibana, K. Tachibana, T. Yokobori, K. Honma, T. Nakano, T. Asao, R. Kuwahara, K. Aoyama, H. Yasuda, M. Kelly, H. Kuwano and D. Yamanouchi, Int J Oncol, 2018, 52, 679-686.

214. , !!! INVALID CITATION !!! 199, 201.

215. M. Hirai, S. Ajito, K. Takahashi, T. Iwasa, X. Li, D. Wen, R. Kawai-Hirai, N. Ohta, N. Igarashi and N. Shimizu, The Journal of Physical Chemistry B, 2019, 123, 3421-3429.

216. V. Kotaidis, C. Dahmen, G. von Plessen, F. Springer and A. Plech, The Journal of chemical physics, 2006, 124, 184702.

217. L. Zhou, X. Wang, H.-J. Shin, J. Wang, R. Tai, X. Zhang, H. Fang, W. Xiao, L. Wang, C. Wang, X. Gao, J. Hu and L. Zhang, Journal of the American Chemical Society, 2020, 142, 55835593.
218.

V. Gnyawali, J.-Z. Wang, Y. Wang, G. Fishbein, L. So, E. Abenojar, A. de Leon, A. Exner, S. Tsai and M. Kolios, Individual nanobubbles detection using acoustic based flow cytometry, 2019.

219. R. W. DeBlois and C. P. Bean, Review of Scientific Instruments, 1970, 41, 909-916.

220. C. Hernandez, E. C. Abenojar, J. Hadley, A. C. de Leon, R. Coyne, R. Perera, R. Gopalakrishnan, J. P. Basilion, M. C. Kolios and A. A. Exner, Nanoscale, 2019, 11, 851-855.

221. Y. Oda, R. Suzuki, T. Mori, H. Takahashi, H. Natsugari, D. Omata, J. Unga, H. Uruga, M. Sugii, S. Kawakami, Y. Higuchi, F. Yamashita, M. Hashida and K. Maruyama, Int J Pharm, 2015, 487.

222. T. H. Yin, P. Wang, R. Q. Zheng, B. W. Zheng, D. Cheng, X. L. Zhang and X. T. Shuai, Int J Nanomed, 2012, 7, 895-904.

223. X. Z. Fan, L. F. Wang, Y. L. Guo, H. P. Tong, L. Li, J. Ding and H. Y. Huang, Nanotechnology, 2013, 24.

224. H. P. Tong, L. F. Wang, Y. L. Guo, L. Li, X. Z. Fan, J. Ding and H. Y. Huang, Ultrasound Med Biol, 2013, 39, 2147-2157.

225. M. Kitano, M. Kudo, K. Yamao, T. Takagi, H. Sakamoto, T. Komaki, K. Kamata, H. Imai, Y. Chiba, M. Okada, T. Murakami and Y. Takeyama, Am J Gastroenterol, 2012, 107, 303-310.

226. F. Prada, A. Perin, A. Martegani, L. Aiani, L. Solbiati, M. Lamperti, C. Casali, F. Legnani, L. Mattei, A. Saladino, M. Saini and F. DiMeco, Neurosurgery, 2014, 74, 542-552. J. J. Zhang, L. S. Wei and Y. L. Zhao, 3 Biotech, 2020, 10. V. Paefgen, D. Doleschel and F. Kiessling, Front Pharmacol, 2015, 6, 197.

229. M. A. Borden, D. E. Kruse, C. F. Caskey, S. K. Zhao, P. A Dayton and K. W. Ferrara, leee T Ultrason Ferr, 2005, 52, 1992-2002.

230. K. Ferrara, R. Pollard and M. Borden, Annu Rev Biomed Eng, 2007, 9, 415-447

231. N. Rapoport, WIREs Nanomedicine and Nanobiotechnology, 2012, 4, 492-510.

232. P. S. Sheeran and P. A. Dayton, Curr Pharm Design, 2012 18, 2152-2165.

233. B. E. O'Neill and N. Rapoport, Ther Deliv, 2011, 2, 11651187.

234

K. E. Wilson, T. Y. Wang and J. K. Willmann, J Nucl Med, 2013, 54, 1851-1854.

235. K. Tamarov, A. Sviridov, W. Xu, M. Malo, V. Andreev, V. Timoshenko and V.-P. Lehto, Acs Appl Mater Inter, 2017, 9, 35234-35243.

236. R. P. Bagwe, L. R. Hilliard and W. Tan, Langmuir, 2006, 22, 4357-4362.

237. A. de Leon, R. Perera, C. Hernandez, M. Cooley, O. Jung, S. Jeganathan, E. Abenojar, G. Fishbein, A. J. Sojahrood, C. C. Emerson, P. L. Stewart, M. C. Kolios and A. A. Exner, Nanoscale, 2019, 11, 15647-15658.

238. F. Cavalieri, I. Finelli, M. Tortora, P. Mozetic, E. Chiessi, F. Polizio, T. B. Brismar and G. Paradossi, Chemistry of Materials, 2008, 20, 3254-3258.

239. B. Cerroni, E. Chiessi, S. Margheritelli, L. Oddo and G. Paradossi, Biomacromolecules, 2011, 12, 593-601.

240. V. Sanna, G. Pintus, P. Bandiera, R. Anedda, S. Punzoni, B. Sanna, V. Migaleddu, S. Uzzau and M. Sechi, Mol Pharmaceut, 2011, 8, 748-757.

241. Y. Ding, Q. Cao, S. Qian, X. Chen, Y. Xu, J. Chen and H. Shen, Journal of Ultrasound in Medicine, 2020, 39, 761-773. 
242. R. H. Perera, A. de Leon, X. Wang, Y. Wang, G. Ramamurthy, P. Peiris, E. Abenojar, J. P. Basilion and A. A. Exner, Nanomedicine: Nanotechnology, Biology and Medicine, 2020, 28, 102213.

243. Z. Yu, M. Hu, Z. Li, X. Dan, L. Zhu, Y. Guo, Q. Liu, W. Lan, J. Jiang and L. Wang, Nanotechnology, 2020, 31, 205101.

244. H. Yang, W. Cai, L. Xu, X. Lv, Y. Qiao, P. Li, H. Wu, Y. Yang, L. Zhang and Y. Duan, Biomaterials, 2015, 37, 279-288.

245. X. Zhang, M. Wu, Y. Zhang, J. Zhang, J. Su and C. Yang Colloids Surf B Biointerfaces, 2020, 189, 110861.

246. I. Steinberg, D. M. Huland, O. Vermesh, H. E. Frostig, W. S. Tummers and S. S. Gambhir, Photoacoustics, 2019, 14, 77 98.

247. E. Huynh, J. F. Lovell, B. L. Helfield, M. Jeon, C. Kim, D. E. Goertz, B. C. Wilson and G. Zheng, Journal of the American Chemical Society, 2012, 134, 16464-16467.

248. C. Kim, R. Qin, J. S. Xu, L. V. Wang and R. Xu, J Biomed Opt, 2010, 15, 010510.

249. F. J. Bodera, M. J. Moore, Y. Wang, A. C. D. Leon, E. Abenojar, A. A. Exner and M. C. Kolios, 2018.

250. V. P. Zharov, Nat Photonics, 2011, 5, 110-116.

251. M. Sarimollaoglu, D. A. Nedosekin, Y. A. Menyaev, M. A. Juratli and V. P. Zharov, Photoacoustics, 2014, 2, 1-11.

252. E. I. Galanzha, R. Weingold, D. A. Nedosekin, M. Sarimollaoglu, J. Nolan, W. Harrington, A. S. Kuchyanov, R. G. Parkhomenko, F. Watanabe, Z. Nima, A. S. Biris, A. I. Plekhanov, M. I. Stockman and V. P. Zharov, Nat Commun, 2017, 8, 15528.

253. E. V. Shashkov, M. Everts, E. I. Galanzha and V. P. Zharov, Nano Letters, 2008, 8, 3953-3958.

254. E. I. Galanzha, E. V. Shashkov, P. M. Spring, J. Y. Suen and V. P. Zharov, Cancer Res, 2009, 69, 7926-7934.

255. J. W. Kim, E. I. Galanzha, E. V. Shashkov, H. M. Moon and V. P. Zharov, Nat Nanotechnol, 2009, 4, 688-694.

256. E. I. Galanzha, E. Shashkov, M. Sarimollaoglu, K. E. Beenken, A. G. Basnakian, M. E. Shirtliff, J. W. Kim, M. S. Smeltzer and V. P. Zharov, Plos One, 2012, 7.

257. M. V. Khodakovskaya, K. de Silva, D. A. Nedosekin, E. Dervishi, A. S. Biris, E. V. Shashkov, E. I. Galanzha and V. P. Zharov, P Natl Acad Sci USA, 2011, 108, 1028-1033.

258. Y. Lin, Z. Y. Chen and F. Yang, Curr Pharm Design, 2013, 19, 3342-3351.

259. J. Ma, C. S. Xu, F. Gao, M. Chen, F. Li and L. F. Du, Mol Med Rep, 2015, 12, 4022-4028.

260. L. E. Andrews, M. H. Chan and R. S. Liu, Nanotechnology, 2019, 30, 182001.

261. J. Li, Z. Feng, N. Gu and F. Yang, Journal of Materials Science \& Technology, 2020, DOI: https://doi.org/10.1016/j.jmst.2020.02.045.

262. P. Nittayacharn, H. X. Yuan, C. Hernandez, P. Bielecki, H. Zhou and A. A. Exner, Journal of pharmaceutical sciences, 2019, 108, 3091-3098.

263. X. Zhou, L. Guo, D. Shi, S. Duan and J. Li, Nanoscale Res Lett, 2019, 14, 24.

264. P. Nittayacharn, E. Abenojar, A. De Leon, D. Wegierak and A. A. Exner, Front Pharmacol, 2020, 11.

265. W. Lin, X. Xie, J. Deng, H. Liu, Y. Chen, X. Fu, H. Liu and Y. Yang, J Drug Target, 2016, 24, 134-146.

266. X. Xie, W. Lin, H. Liu, J. Deng, Y. Chen, H. Liu, X. Fu and Y. Yang, Drug Deliv, 2016, 23, 2756-2764.

267. S. S. Thakur, Y.-S. Chen, Z. H. Houston, N. Fletcher, N. L. Barnett, K. J. Thurecht, I. D. Rupenthal and H. S. Parekh,
European Journal of Pharmaceutics and Biopharmaceutics, 2019, 136, 102-107.

268. S. Zhong, Z. Ling, Z. Zhou, J. He, H. Ran, Z. Wang, Q. Zhang, W. Song, Y. Zhang and J. Luo, Pharmaceutical Development and Technology, 2020, 25, 454-463.

269. E. Y. Lukianova-Hleb, X. Ren, J. A. Zasadzinski, X. Wu and D. O. Lapotko, Adv Mater, 2012, 24, 3831-3837.

270. E. Y. Lukianova-Hleb, X. Ren, R. R. Sawant, X. Wu, V. P. Torchilin and D. O. Lapotko, Nature Medicine, 2014, 20, 778-784.

271. R. Suzuki, T. Takizawa, Y. Negishi, N. Utoguchi, K. Sawamura, K. Tanaka, E. Namai, Y. Oda, Y. Matsumura and K. Maruyama, Journal of controlled release : official journal of the Controlled Release Society, 2008, 125, 137-144.

272. S. Horie, Y. Watanabe, M. Ono, S. Mori and T. Kodama, Cancer Science, 2011, 102, 2082-2089.

273.

T. Yin, P. Wang, J. Li, R. Zheng, B. Zheng, D. Cheng, R. Li, J. Lai and X. Shuai, Biomaterials, 2013, 34, 4532-4543.

274.

T. Yin, P. Wang, J. Li, Y. Wang, B. Zheng, R. Zheng, D. Cheng and X. Shuai, Biomaterials, 2014, 35, 5932-5943.

275. L. F. Wang, M. Zhang, K. B. Tan, Y. L. Guo, H. P. Tong, X. Z. Fan, K. J. Fang and R. Li, Plos One, 2014, 9.

276. M. Wu, H. Zhao, L. Guo, Y. Wang, J. Song, X. Zhao, C. Li, L. Hao, D. Wang and J. Tang, Drug Deliv, 2018, 25, 226-240.

277. J. Baumgart, L. Humbert, E. Boulais, R. Lachaine, J. J. Lebrun and M. Meunier, Biomaterials, 2012, 33, 2345-2350.

278. E. Y. Lukianova-Hleb, D. S. Wagner, M. K. Brenner and D. O. Lapotko, Biomaterials, 2012, 33, 5441-5450.

J. C. Fraire, G. Houthaeve, J. Liu, L. Raes, L. Vermeulen, S. Stremersch, T. Brans, G. García-Díaz Barriga, S. De Keulenaer, F. Van Nieuwerburgh, R. De Rycke, J. Vandesompele, P. Mestdagh, K. Raemdonck, W. H. De Vos, S. De Smedt and K. Braeckmans, J Control Release, 2020, 319, 262-275.

L. Raes, S. Stremersch, J. Fraire, T. Brans, G. Goetgeluk, S. De Munter, L. Van Hoecke, R. Verbeke, J. Van Hoeck, R. Xiong, X. Saelens, B. Vandekerckhove, S. De Smedt, K. Raemdonck and K. Braeckmans, Nano-Micro Letters, 2020, 12.

281. E. Teirlinck, R. Xiong, T. Brans, K. Forier, J. Fraire, H. Van Acker, N. Matthijs, R. De Rycke, S. C. De Smedt, T. Coenye and K. Braeckmans, Nat Commun, 2018, 9, 4518.

282. E. Teirlinck, A. Barras, J. Liu, J. C. Fraire, T. Lajunen, R. Xiong K. Forier, C. Li, A. Urtti, R. Boukherroub, S. Szunerits, S. C. De Smedt, T. Coenye and K. Braeckmans, Pharmaceutics, 2019, 11.

283. E. Teirlinck, J. C. Fraire, H. Van Acker, J. Wille, R Swimberghe, T. Brans, R. Xiong, M. Meire, R. J. G. De Moor, S. C. De Smedt, T. Coenye and K. Braeckmans, Biofilm, 2019, 1, 100004.

284. S. M. Fix, M. A. Borden and P. A. Dayton, Journal of controlled release : official journal of the Controlled Release Society, 2015, 209, 139-149.

285. R. Cavalli, M. Soster and M. Argenziano, Ther Deliv, 2016, 7, 117-138.

286. R. Cavalli, A. Bisazza, A. Rolfo, S. Balbis, D. Madonnaripa, I. Caniggia and C. Guiot, Int J Pharm, 2009, 378, 215-217.

287. P. Bhandari, G. Novikova, C. J. Goergen and J. Irudayaraj, Sci Rep-Uk, 2018, 8, 3112.

288. E. Ficiarà, S. A. Ansari, M. Argenziano, L. Cangemi, C. Monge, R. Cavalli and F. D'Agata, Molecules, 2020, 25. 
289. C. Magnetto, M. Prato, A. Khadjavi, G. Giribaldi, I. Fenoglio, J. Jose, G. R. Gulino, F. Cavallo, E. Quaglino, E. Benintende, G. Varetto, A. Troia, R. Cavalli and C. Guiot, RSC Advances, 2014, 4, 38433-38441.

290. G. Banche, M. Prato, C. Magnetto, V. Allizond, G. Giribaldi, M. Argenziano, A. Khadjavi, G. R. Gulino, N. Finesso, N. Mandras, V. Tullio, R. Cavalli, C. Guiot and A. M. Cuffini, Future Microbiol, 2015, 10, 929-939.

291. B. Cheng, C. Bing, Y. Xi, B. Shah, A. A. Exner and R. Chopra, Ultrasound in medicine \& biology, 2019, 45, 2174-2187.

292. E. Y. Lukianova-Hleb, Y.-S. Kim, I. Belatsarkouski, A. M. Gillenwater, B. E. O'Neill and D. O. Lapotko, Nat Nanotechnol, 2016, 11, 525-532.

293. F. Sauvage, J. C. Fraire, K. Remaut, J. Sebag, K. Peynshaert, M. Harrington, F. J. Van de Velde, R. Xiong, M.-J. Tassignon, T. Brans, K. Braeckmans and S. C. De Smedt, Acs Nano, 2019, 13, 8401-8416.

294. A. Koşar, M. Şeşen, O. Oral, Z. Itah and D. Gozuacik, IEEE Trans Biomed Eng, 2011, 58, 1337-1346.

295. Z. Itah, O. Kutlu, O. Perk, M. Sesen, E. Demir, S. Erbil, A. I. Doğan Ekici, S. Ekici, A. Kosar and D. Gozuacik, Experimental biology and medicine (Maywood, N.J.), 2013, 238.

296. O. Y. Perk, M. Şeşen, D. Gozuacik and A. Koşar, Annals of Biomedical Engineering, 2012, 40, 1895-1902.

297. M. Ghorbani, C. Sözer, G. Alcan, M. Unel, S. Ekici, H. Uvet and A. Kosar, AIP Advances, 2018, 8, 035108.

298. B. Dollet, P. Marmottant and V. Garbin, Annu Rev Fluid Mech, 2019, 51, 331-355.

299. Z. Cao, W. Li, R. Liu, X. Li, H. Li, L. Liu, Y. Chen, C. Lv and Y. Liu, Biomedicine \& Pharmacotherapy, 2019, 118, 109340.

300. M. G. Shapiro, P. W. Goodwill, A. Neogy, M. Yin, F. S. Foster D. V. Schaffer and S. M. Conolly, Nat Nanotechnol, 2014, 9, 311-316.

301. A. Lakshmanan, A. Farhadi, S. P. Nety, A. Lee-Gosselin, R. W. Bourdeau, D. Maresca and M. G. Shapiro, Acs Nano, 2016, 10, 7314-7322.

302. R. W. Bourdeau, A. Lee-Gosselin, A. Lakshmanan, A. Farhadi, S. R. Kumar, S. P. Nety and M. G. Shapiro, Nature, 2018, 553, 86-90.

303. G. H. Wang, L. Song, X. D. Hou, S. Kala, K. F. Wong, L. Y. Tang, Y. L. Dai and L. Sun, Biomaterials, 2020, 236. 